\title{
Highlights from BNL-RHIC 2011-2013
}

\author{
M. J. Tannenbaum * \\ Physics Department, 510c, \\ Brookhaven National Laboratory, \\ Upton, NY 11973-5000, USA \\ mjt@bnl.gov
}

October 15, 2018

\begin{abstract}
Highlights from Brookhaven National Laboratory (BNL) and experiments at the BNL Relativistic Heavy Ion Collider (RHIC) are presented for the years 2011-2013. This review is a combination of lectures which discussed the latest results each year at a three year celebration of the 50th anniversary of the International School of Subnuclear Physics in Erice, Sicily, Italy. Since the first collisions in the year 2000, RHIC has provided nucleus-nucleus and polarized proton-proton collisions over a range of nucleon-nucleon c.m. energies $\left(\sqrt{s_{N N}}\right)$ from 7.7 to 510 $\mathrm{GeV}$ with nuclei from deuterium to uranium, most often gold. The objective was the discovery of the Quark Gluon Plasma, which was achieved, and the measurement of its properties, which were much different than expected, namely a 'perfect fluid' of quarks and gluons with their color charges exposed rather than a gas. Topics including quenching of light and heavy quarks at large transverse momentum, thermal photons, search for a QCD critical point as well as measurements of collective flow, two-particle correlations and $J / \Psi$ suppression are presented. During this period, results from the first and subsequent heavy ion measurements at the Large Hadron Collider (LHC) at CERN became available. These confirmed and extended the RHIC discoveries and have led to ideas for new and improved measurements.
\end{abstract}

Keywords: RHIC; LHC; sQGP; heavy ion collisions; jet quenching; thermal radiation; collective flow; QCD critical point; Cumulants; $J / \Psi$ suppression; deconfinement .

PACS numbers:25.75.-q

\section{Contents}

1 Introduction 2

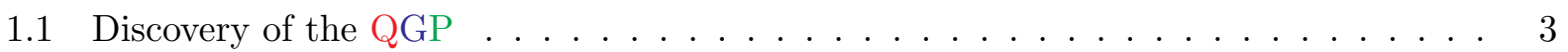

2 Progress at BNL and the RHIC machine 2011-2013. 4

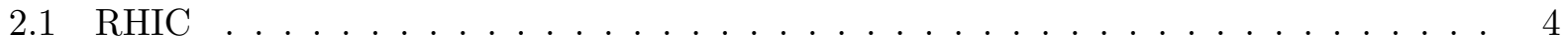

2.2 BNL's Superconducting Magnet Division . . . . . . . . . . . . . 6 6

2.3 National Synchrotron Light Source-II . . . . . . . . . . . . . . . . . . 8

2.4 BNL g-2 Magnet moves to FERMILAB . . . . . . . . . . . . . . . . . . . . 8

${ }^{*}$ Research supported by U. S. Department of Energy, DE-AC02-98CH10886. 
4 Introduction to QGP Physics 11

$4.1 J / \Psi$ suppression — the original "gold-plated" QGP signature . . . . . . . . . . . 11

4.2 Detector issues in $\mathrm{A}+\mathrm{A}$ compared to $\mathrm{p}-\mathrm{p}$ collisions $\ldots \ldots \ldots \ldots \ldots$

5 Collective Flow 15

5.1 Two-Particle Correlations and Flow . . . . . . . . . . . . . 16

5.2 Triangular flow odd harmonics . . . . . . . . . . . . . . 19

6 RHIC beam energy scan - In search of the critical point 20

6.1 A press release during the 2011 school . . . . . . . . . . . . . . . . 21

6.2 The future measurement has appeared without a press release. . . . . . . . . 23

7 Hard Scattering as an in-situ probe $\quad 24$

7.1 Jet quenching - suppression of high $p_{T}$ particles . . . . . . . . . . . 25

7.2 Two-Particle Correlations and Fragmentation Functions . . . . . . . . . . . 28

7.3 Two-Particle Correlations and Jet Imbalance . . . . . . . . . . . . . . . . . 30

8 Anisotropic flow $\left(v_{2}\right)$ of direct- $\gamma$

9 A charming surprise $\quad 33$

10 Do latest $J / \Psi$ results from ALICE prove deconfinement?

11 Surprises in $\mathrm{d}+\mathrm{Au}$ and $\mathrm{p}+\mathrm{Pb}$ measurements 38

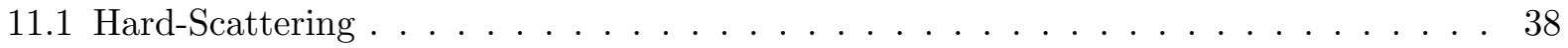

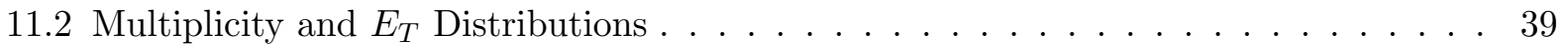

11.3 Collective Flow in $\mathrm{d}+\mathrm{Au}($ and $\mathrm{p}+\mathrm{Pb})$ collisions? . . . . . . . . . . . 41

12 The Future

\section{Introduction}

High energy nucleus-nucleus collisions provide the means of creating nuclear matter in conditions of extreme temperature and density [1, 2, 3. The kinetic energy of the incident projectiles would be dissipated in the large volume of nuclear matter involved in the reaction. At large energy or baryon densities, a phase transition is expected from a state of nucleons containing confined quarks and gluons to a state of "deconfined" (from their individual nucleons) quarks and gluons, in chemical and thermal equilibrium, covering a volume that is many units of the confining length scale. This state of nuclear matter was originally given the name Quark Gluon Plasma (QGP) [4], a plasma being an ionized gas.

A typical proposed phase diagram of nuclear matter is shown in Fig. 1 together with the idealized trajectories of the evolution of the medium for $\mathrm{Au}+\mathrm{Au}$ collisions at c.m. energies proposed for the Beam Energy Scan at RHIC in search for a QCD critical point. The bursts represent the hottest and densest stage of the medium when thermal equilibrium is reached shortly after the collision. The axes are the temperature $T$ vs. the baryon chemical potential $\mu_{B}$. The temperature for the transition from the Quark Gluon Plasma (QGP) to a hadron gas is taken as $170 \mathrm{MeV}$ for $\mu_{B}=0$ 


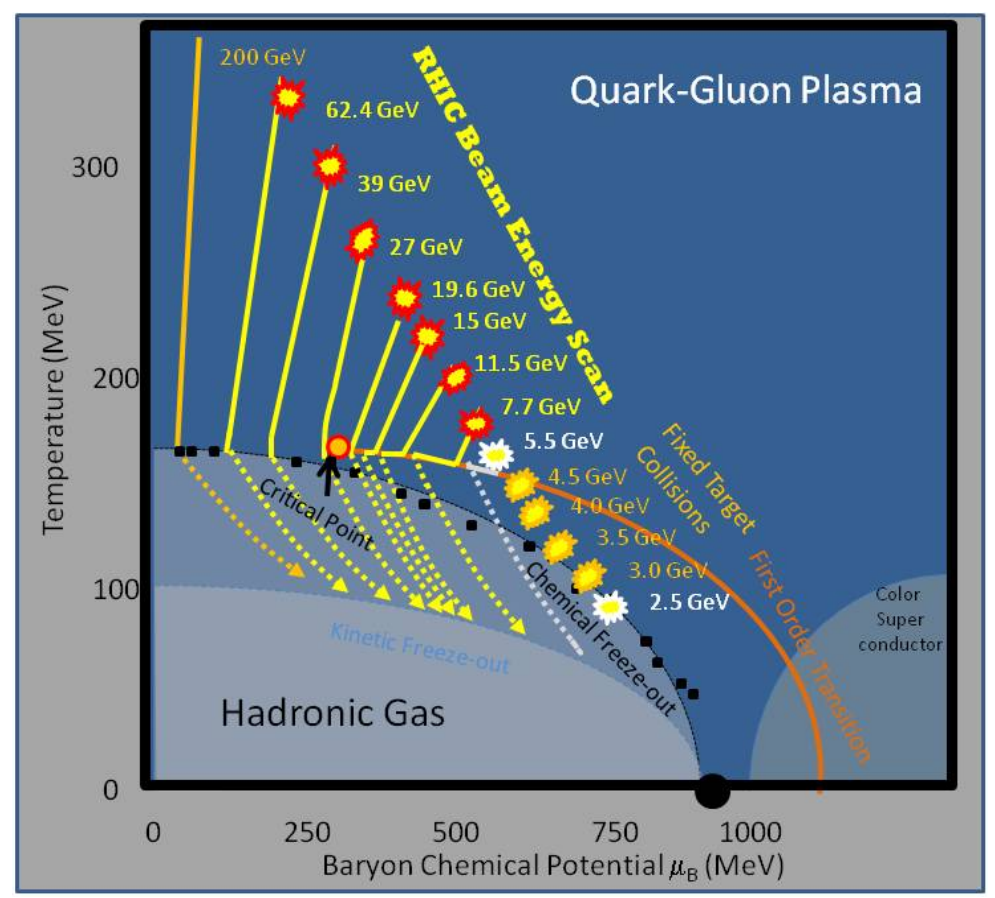

Figure 1: (left) A proposed phase diagram for nuclear matter from STAR Beam Use Request for 2014-2015: Temperature, $T$, vs Baryon Chemical Potential, $\mu_{B}$.

and the phase boundary is predicted to be a smooth crossover down to a critical point below which the phase boundary becomes a first order phase transition.

\subsection{Discovery of the QGP}

The QGP was discovered at RHIC, and announced on April 19, 2005. However the results at RHIC [2] indicated that instead of behaving like a gas of free quarks and gluons, the matter created in heavy ion collisions at nucleon-nucleon c.m. energy $\sqrt{s_{N N}}=200 \mathrm{GeV}$ appears to be more like a liquid. This matter interacts much more strongly than originally expected, as elaborated in peer reviewed articles by the 4 RHIC experiments [5, 6, 7, 8], which inspired the theorists [9] to give it the new name "sQGP" (strongly interacting QGP). These properties were quite different from the "new state of matter" claimed in a press-conference [10] by the CERN fixed target heavy ion program on February 10, 2000, which was neither peer-reviewed nor published.

In spite of not being published, the CERN press-release had a major effect on the press in the United States resulting in an article on the front page of the New York Times [11. Ironically, on this very same front page was an article announcing that the true version of the famous Italian sausage, Mortadella, would, for the first time, be allowed to be imported into the United States. A photograph of the iconic Bologna sausages appeared right next to the article about the CERN "qgp". Unfortunately, the first European Baloney to arrive in the U. S. was the CERN announcement. 1

\footnotetext{
${ }^{1}$ It is important for the reader of these proceedings to be aware that a high official of CERN was in the audience during this talk and made no objection to this comment. Furthermore, the author has a long, positive and productive relationship with this great laboratory and has praised its many successes. Thus he feels justified in commenting on one of their rare misjudgments. See Ref. [3] for a detailed scientific discussion.
} 


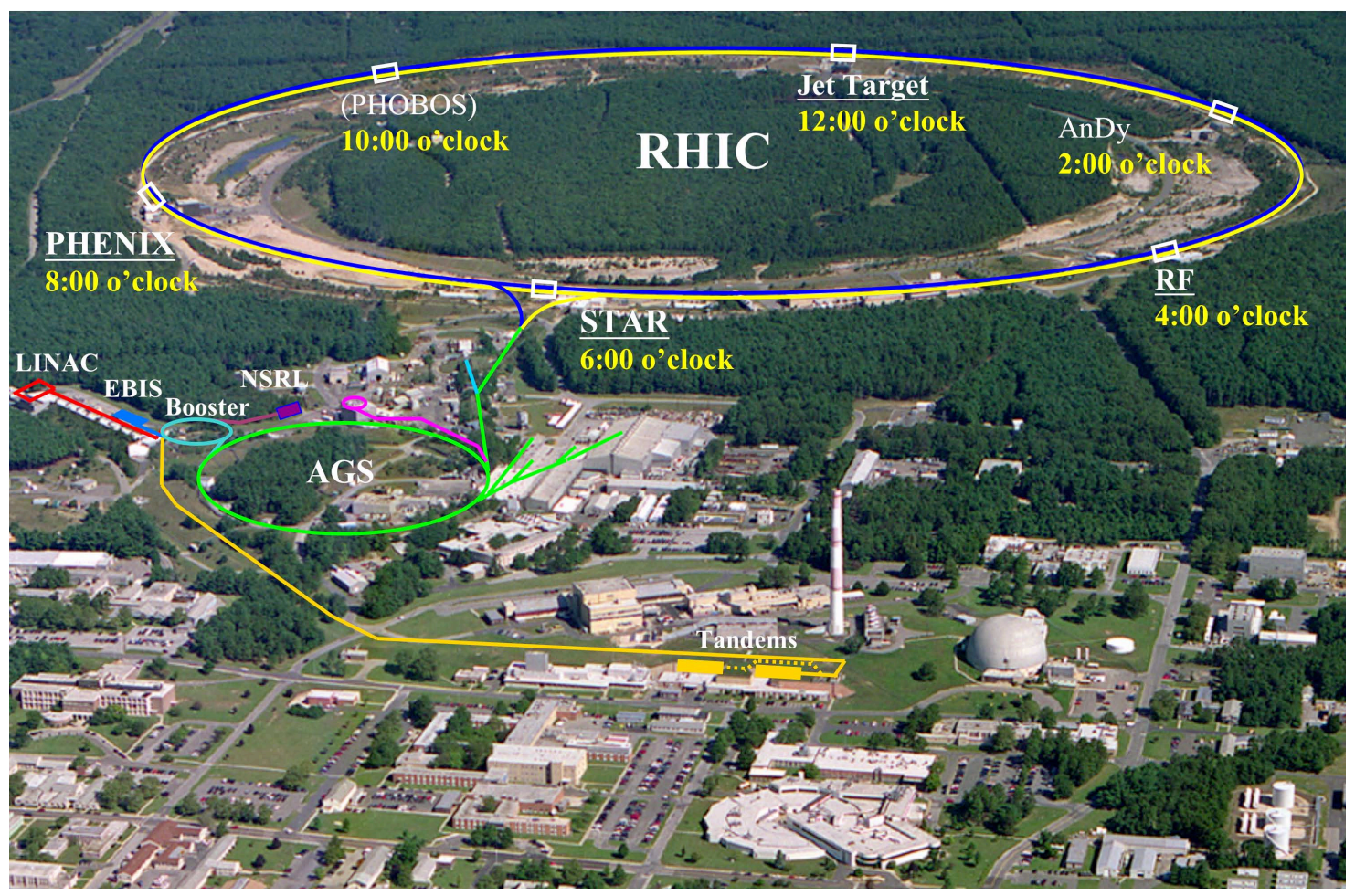

Figure 2: Aerial view of the RHIC facility [17]. The six crossing points are labelled as on a clock. The two principal experiments still running are PHENIX and STAR. Two smaller experiments PHOBOS [6] and BRAHMS [5] have been completed; a test run, AnDy, occupies the former location of BRAHMS. The LINAC is the injector for polarized protons into the Booster/AGS/RHIC chain; with the Jet Target used for precision beam polarization measurements. The TANDEM injector for Ions has been replaced by the Electron Beam Ion Source (EBIS) starting with the 2012 run.

However the situation at CERN improved dramatically in November 2010 with the startup of $\mathrm{Pb}+\mathrm{Pb}$ collisions in the CERN-LHC at $\sqrt{s_{N N}}=2760 \mathrm{GeV}(2.76 \mathrm{TeV})$ where the real QGP was observed. The LHC Pb+Pb measurements confirm the RHIC discoveries [12, 13, 14] and add some new information - notably with fully reconstructed jets [15, 16].

\section{Progress at BNL and the RHIC machine 2011-2013.}

\section{$2.1 \quad$ RHIC}

With the shutdown of the Tevatron at FERMILAB, on September 30, 2011 after 28 years of operation, the Relativistic Heavy Ion Collider (RHIC) at Brookhaven National Laboratory (Fig. 2) is the only hadron collider in the U.S. and one of only two hadron-colliders in the world, the other being the CERN-LHC. RHIC is also the world's first and only polarized proton collider. RHIC is composed of two independent rings, of circumference $3.8 \mathrm{~km}$, containing a total of 1,740 superconducting magnets(see Fig. 4a, below). RHIC can collide any species with any other species and since beginning operation in the year 2000 has provided collisions at 13 different values of nucleon-nucleon c.m. energy, $\sqrt{s_{N N}}$, and nine different species combinations including $\mathrm{Au}+\mathrm{Au}$, $\mathrm{d}+\mathrm{Au}, \mathrm{Cu}+\mathrm{Cu}, \mathrm{Cu}+\mathrm{Au}, \mathrm{U}+\mathrm{U}$, if differently polarized protons are counted as different species. For the runs in 2010-2011, an Au+Au energy scan was performed with $\sqrt{s_{N N}}=7.7,11.5,19.6,27,39$, 62.4, $200 \mathrm{GeV}$. The performance history of RHIC with A+A and polarized p-p collisions is shown 
in Fig. 3 .
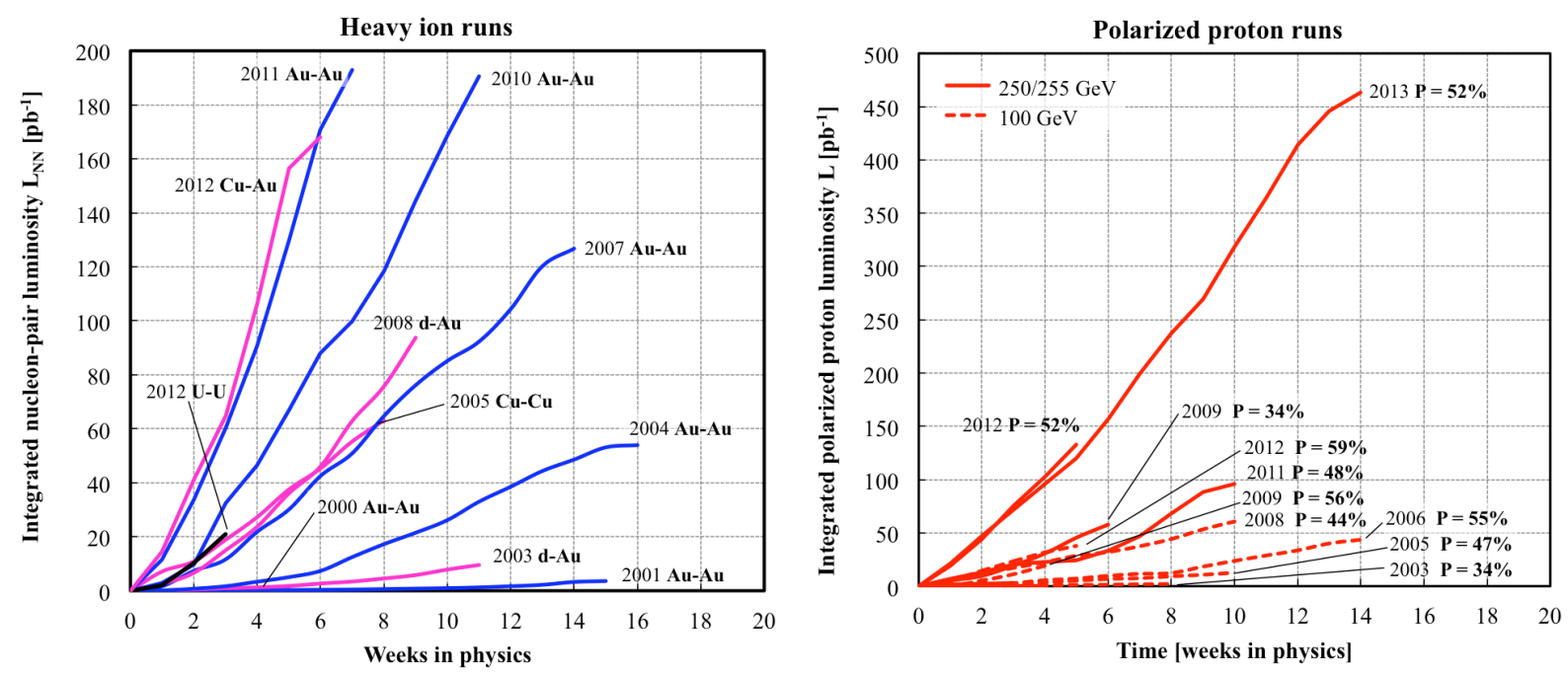

Figure 3: a)(left) $\mathrm{Au}+\mathrm{Au}$ performance, where the nucleon-pair luminosity is defined as $L_{\mathrm{NN}}=$ $A \times B \times L$, where $L$ is the luminosity and $A, B$ are the number of nucleons in the colliding species. b) (right) Polarized p-p performance. Courtesy Wolfram Fischer.

At present RHIC operates at 15 times design luminosity for $\mathrm{Au}+\mathrm{Au}$ and has shown a factor 2 progress in integrated luminosity $\mathcal{L}_{\text {int }}$ per week from Run-4 (2004) to Run-7 to Run-10 to Run-11. In 2011, 3 dimensional stochastic cooling (with only 2-d transverse cooling active) was introduced for the $\mathrm{Au}+\mathrm{Au}$ collisions to improve the storage lifetime. Further improvements in the longitudinal profile, i.e. smaller diamond size without bucket migration, will be made with increased longitudinal focusing from new $56 \mathrm{MHz}$ radio frequency storage cavity.

A significant improvement to the A+A program starting with Run-12 in 2012 was the replacement of the 40 year old Tandem Van de Graaff injector with an Electron Beam Ion Source (EBIS). A 10 A electron beam creates the desired charge state(s) in a trap within a $5 \mathrm{~T}$ superconducting solenoid. This is then accelerated through the Radio Frequency Quadrupole (RFQ) and linac and injected into the AGS Booster (Fig. 2). All ion species including noble gases, uranium and polarized ${ }^{3} \mathrm{He}$ are available. Commissioning of the EBIS started during early 2011 and supplied $\mathrm{He}^{+}$, $\mathrm{He}^{2+}, \mathrm{Ne}^{5+}, \mathrm{Ne}^{8+}, \mathrm{Ar}^{11+}, \mathrm{Ti}^{18+}$ and $\mathrm{Fe}^{20+}$ for the NASA Space Radiation Research Laboratory (NSRL) at BNL [18]. For the first time in a collider, $\mathrm{Cu}+\mathrm{Au}$ and $\mathrm{U}+\mathrm{U}$ collisions at $\sqrt{s_{N N}}=200$ $\mathrm{GeV}$ were studied. Also, the polarized p-p runs in 2012 included both $\sqrt{s}=200$ and $510 \mathrm{GeV}$ with improved polarization and luminosity for the purposes of comparison data for the new silicon vertex detectors introduced $(200 \mathrm{GeV})$ and for measurements of flavor-identified parton spin distribution functions using the parity violating single spin asymmetry in $W^{ \pm}$production $(510 \mathrm{GeV})$ [19]. The performance in Run-12 was even more outstanding than usual thanks to the new EBIS source as well as 3-dimensional stochastic cooling [20] of which the third dimension (horizontal) was active only for the $U+U$ segment at the end of the run.

The 2013 run (Run-13) was devoted entirely to polarized proton-proton collisions at $\sqrt{s_{N N}}=510$ $\mathrm{GeV}$ for the measurement of flavor-identified spin structure functions using parity-violating production of $W^{ \pm}$-bosons. Machine improvements included an upgraded Optically Pumped Polarized $\mathrm{H}^{-}$source (OPPIS) with an order of magnitude increase in beam current to $10 \mathrm{~mA}$ and an increase in polarization from $85 \%$ to $90 \%$. The components of the upgrade are an atomic hydrogen injec- 
tor (in collaboration with BINP, Novosibirsk), a 3 Tesla superconducting solenoid and improved beam diagnostics and polarization measurement. A further improvement which, together with the improved OPPIS, is expected to yield a doubling of the luminosity (which is now limited by the head-on beam-beam effect) is the addition of electron lenses for partial compensation of head-on beam-beam tune shift. The idea is that in addition to the two regions of beam-beam collisions with positively charged beam, add another another collision point with a negatively charged beam with the same amplitude dependence to have the effect from p-e collisions partially cancel the p-p effect. The commissionning of the electron lenses with a new lattice proved to be challenging, so the latter part of the run was done with the lattice tested in 2012 and provided record luminosity and polarizations near $60 \%$.

\subsection{BNL's Superconducting Magnet Division}

All of the upgrades to the RHIC machine mentioned above involved superconducting magnets built or developed in BNL's Superconducting Magnet Division. The Magnet Division also developed the RHIC machine superconducting magnets (Palmer magnet [21]) which are the basis for the other post-Tevatron machines such as HERA and the LHC. The RHIC dipole design (Fig. 4p) is based on a relatively large bore $(80 \mathrm{~mm}$ inner diameter $)$, single-layer "cosine theta" coil, wound from a (partially) keystoned, kapton-insulated, 30-strand Rutherford-type cable, arranged in coil blocks with intervening copper wedges, in order to meet the stringent field quality specifications, and mechanically supported by a laminated, cold steel yoke encased in a stainless steel shell. The shell contains the helium and is also a load bearing part of the assembly.

It is amusing to note that in order to build the RHIC magnets for the purpose of making the QGP and studying its phase diagram, it is important to understand another phase-diagram, that of $\mathrm{Fe}+\mathrm{C}$, i.e. magnet steel (Fig. 5). The $\mathrm{Fe}+\mathrm{C}$ phase diagram is quite complicated with many phases, but is well known; while the proposed phase diagram of nuclear matter (Fig. 1) seems much simpler, probably because it is largely unknown.

BNL's superconducting magnet division is an international resource and actively participates in many projects, several in the news recently. The CERN Courier of March 2011 featured on its
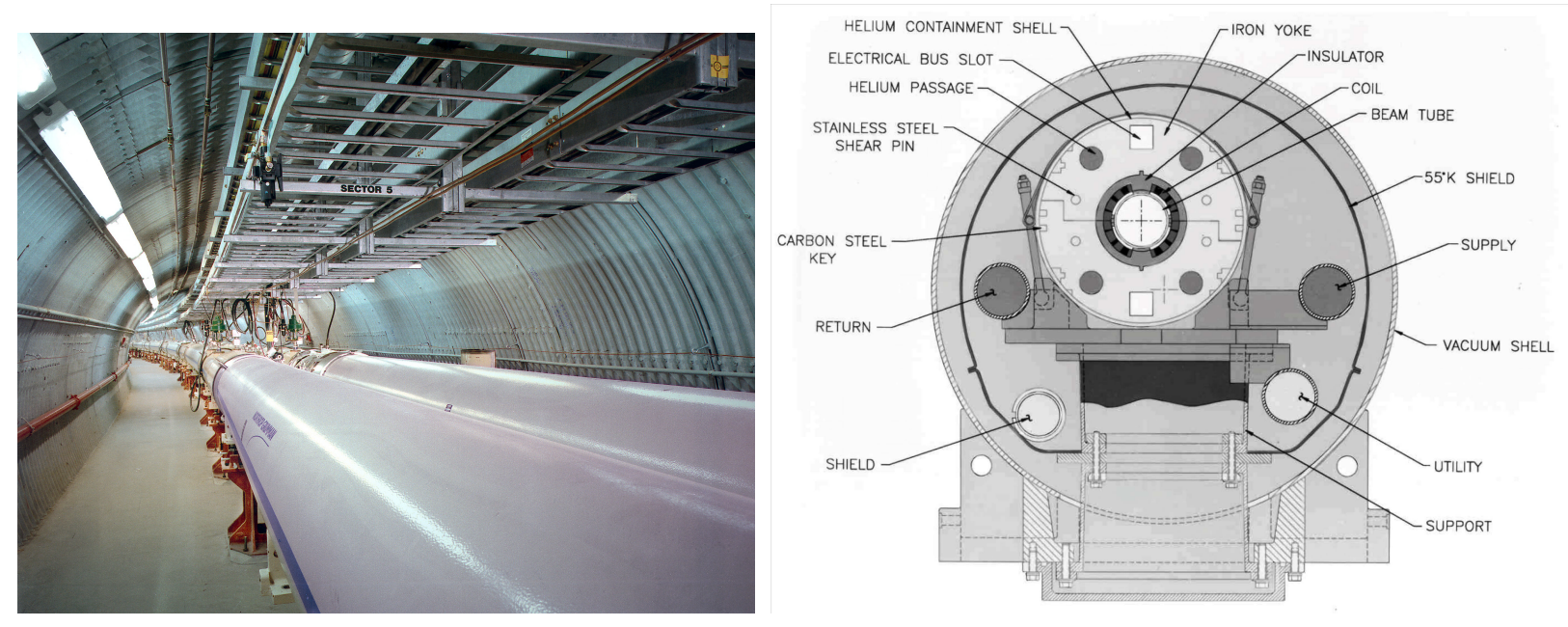

Figure 4: a)(left) Photo of the RHIC machine composed of two independent rings, with a total of 1740 superconducting dipole, quadrupole and corrector magnets. b) (right) Cross section of RHIC dipole. 


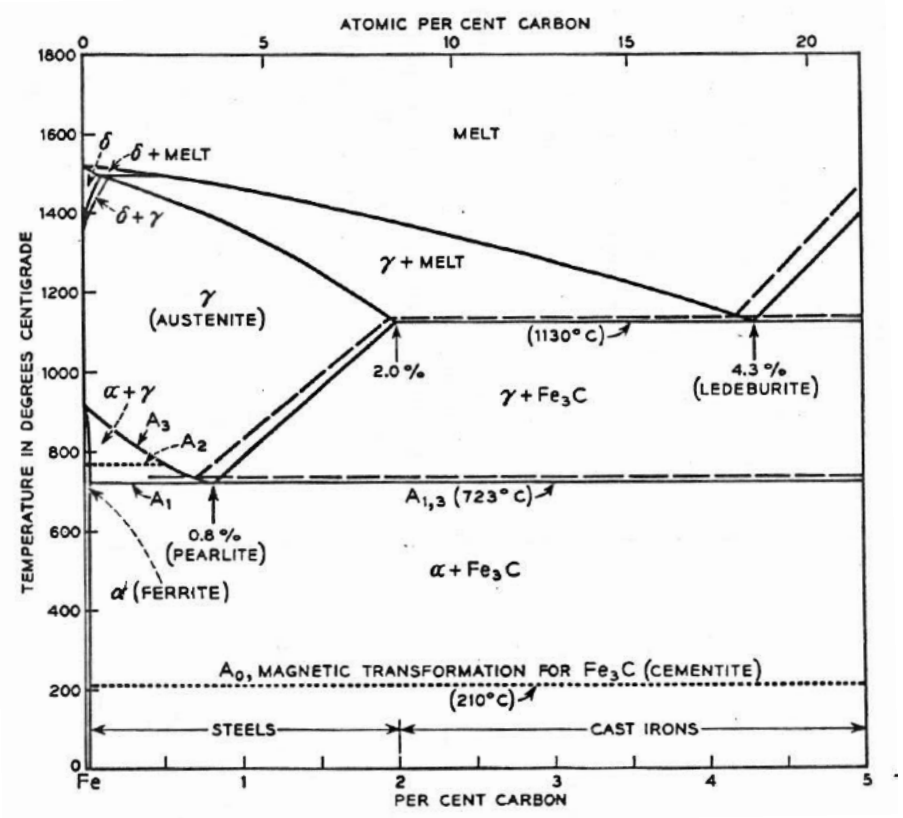

Figure 5: Phase diagram of iron-carbon alloys [22].
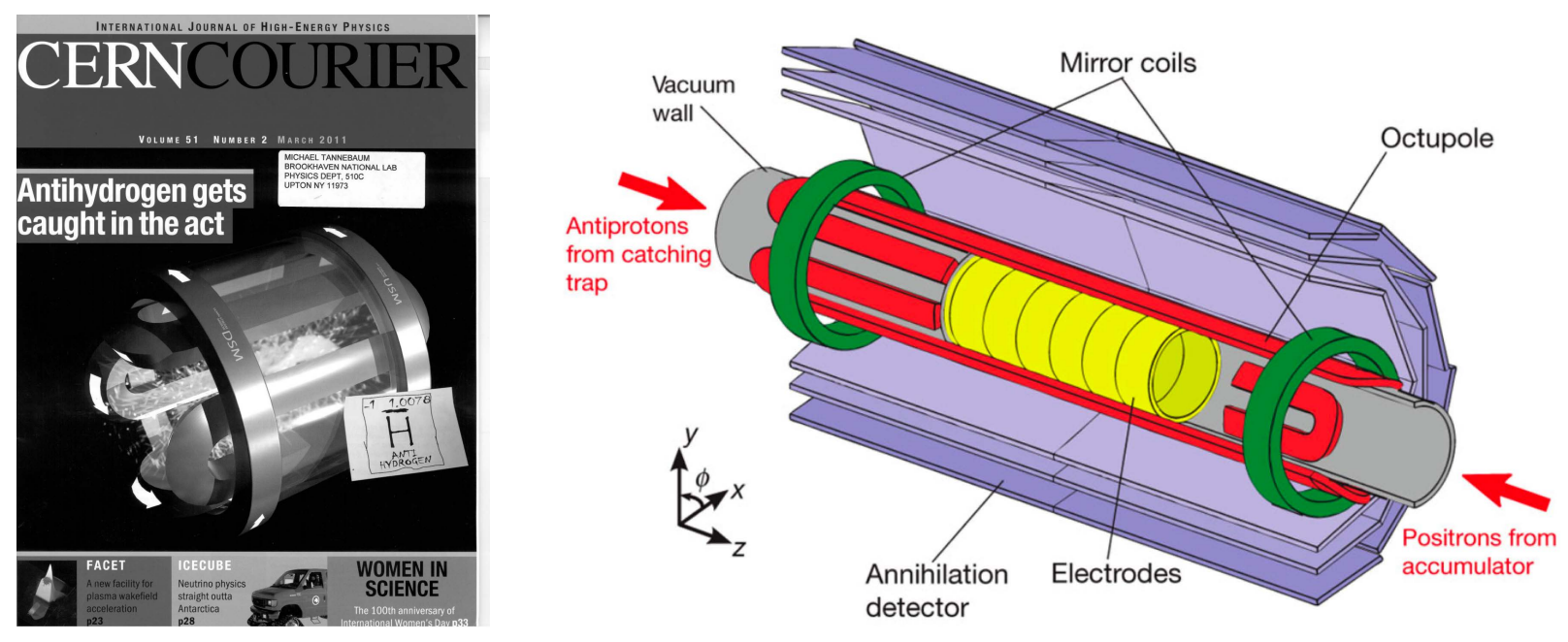

Figure 6: a)(left) CERN Courier cover March 2011. b) (right) Antihydrogen synthesis and trapping region of the ALPHA apparatus [23. The atom-trap magnets, the modular annihilation detector and some of the Penning trap electrodes are shown (not to scale).

cover (Fig. 6a) the beautiful magnet used to trap anti-hydrogen for 1000 seconds in the ALPHA experiment [23] in the Antiproton Decelerator at CERN. The octupole magnet (Fig. 6b) was built by the BNL Magnet Division and provides a very pure octupole field which is zero on the axis and rises sharply near the vacuum wall to keep the anti-hydrogen confined radially.

Another recent press release featuring BNL magnets was the "Indication of Electron Neutrino Appearance" at the T2K (Tokai to Kamioka) Experiment in Japan on June 15, 2011 [24]. BNL provided 5 superconducting dipole corrector magnets in the proton beam at the Japan Proton Ac- 
celerator Research Complex (J-PARC) in Tokai which produced the $\mu$-neutrinos that were detected in the Super-Kamiokande detector after transforming to $e$-neutrinos over the $295 \mathrm{~km}$ flight path.

Other work of the Magnet Division includes: NbTi magnets for the RHIC Electron Lens upgrade; NbTi final focus quadrupole for the ILC; $\mathrm{Nb}_{3} \mathrm{Sn} 11.5$ Tesla strand-test-barrel magnet and two coils for the LHC luminosity upgrade; High Temperature Superconductor (HTS) Quadrupole for the Facility for Rare Isotope Beams at Michigan State University; Spare NbTi dipole for LHC; HTS solenoid R\&D for muon collider and Energy Storage; $\mathrm{Nb}_{3} \mathrm{Sn}$ Open Midplane Dipole for the Muon Collider.

\subsection{National Synchrotron Light Source-II}

In addition to accelerators for high energy particle and nuclear physics, Brookhaven has been innovative in synchrotron radiation light sources. The National Synchrotron Light Source (NSLS) which started operations in 1982 was the first to use the Chasman-Green double-bend achromat lattice [25, which is now the standard lattice at the major synchrotron light sources worldwide. A new third generation light source, NSLS-II, 4.66 times larger in circumference than NSLS, is now under construction at BNL, with unique design features of high brightness, small source size and long beam lines which will replace NSLS in 2014. NSLS-II is designed to deliver photons with high average spectral brightness in the $2 \mathrm{keV}$ to $10 \mathrm{keV}$ energy range exceeding $10^{21} \mathrm{ph} / \mathrm{s} / 0.1 \% \mathrm{BW} / \mathrm{mm}^{2} / \mathrm{mrad}^{2}$. The spectral flux density should exceed $10^{15} \mathrm{ph} / \mathrm{s} / 0.1 \% \mathrm{BW}$ in all spectral ranges. This cuttingedge performance requires the storage ring to support a very high-current electron beam ( $\mathrm{I}=500$ $\mathrm{mA}$ ) with sub-nm-rad horizontal emittance (down to $0.5 \mathrm{~nm}$-rad) and diffraction-limited vertical emittance at a wavelength of $1 \AA$ (vertical emittance of 8 pm-rad) [26].

\subsection{BNL g-2 Magnet moves to FERMILAB}

In June 2013, the $15 \mathrm{~m}$ diameter precision storage ring from the BNL muon $g-2$ experiment [27] began a circuitous $(5000 \mathrm{~km}$ ) very delicate cross-contry trip to Fermilab (only $1440 \mathrm{~km}$ in a straight line) involving custom built trucks and a specially prepared barge which brought the magnet down the East Coast, around the tip of Florida and up the Mississippi River to Illinois. This new muon $g-2$ experiment at Fermilab would be the fifth such experiment, which was pioneered at CERN.

By some incredible coincidence, a few weeks before the ISSP2013 school, the June 2013 CERN Courier reprinted an article from 1970 with the title "Preparing for a third ' $g-2$ '." This brought back good memories to me because I had worked on the second $g-2$ experiment when I was a postdoc at CERN in 1965-66 [28]; but, of course, Prof. Zichichi worked on the ground-breaking original

$g-2$ experiment at CERN in 1959-1961 [29]. My thesis research, done at the BNL-AGS from 1961-64, was muon-proton elastic scattering [30] to find out "Why does the muon weigh heavy?" Even in 2013, with Prof. Higgs in the audience, we still don't know! Other experiments at that time did better: the "two-neutrino experiment" [31] (Nobel Prize) was in the beam to the left of "my" muon beam; while on the right, over the AGS machine, in "inner Mongolia", CP violation [32] was discovered (Nobel Prize). Those were the days; but even more excitement lay ahead.

\section{ICHEP1972: Hard-Scattering, Quarks, and QCD}

At the International Conference on High Energy Physics (ICHEP) in 1972, there were three momentous developments that inform our work today: 
- The discovery in p-p collisions at the CERN ISR of production of particles with large transverse momentum $\left(p_{T}\right)$ which proved that the partons of Deeply Inelastic Scattering (DIS) interacted with each other much more strongly than electromagnetically.

- Measurements of DIS in neutrino scattering presented by Don Perkins who proclaimed that "In terms of constituent models, the fractionally charged (Gell-Mann/Zweig) quark model is the only one which fits both the electron and neutrino data."

- The origin of QCD in the presentation by Harald Fritzsch and Murray Gell-Mann with the title "Current Algebra: Quarks and What Else?"

Figure 7 a shows the first observation of scattering at large $p_{T}$ [33]. The $\pi^{0}$ spectrum breaks away from the $e^{-6 p_{T}}$ dependence known since cosmic ray measurements, with a power-law spectrum that flattens as the c.m energy, $\sqrt{s}$, is increased. Excellent cooperation of experimentalists and theorists showed in 1978 that these data could be explained by QCD [34, 35] if the quarks in a nucleon had "intrinsic" transverse momentum, $k_{T} \approx 1 \mathrm{GeV} / \mathrm{c}$ (Fig. 7b). Although these QCD calculations in
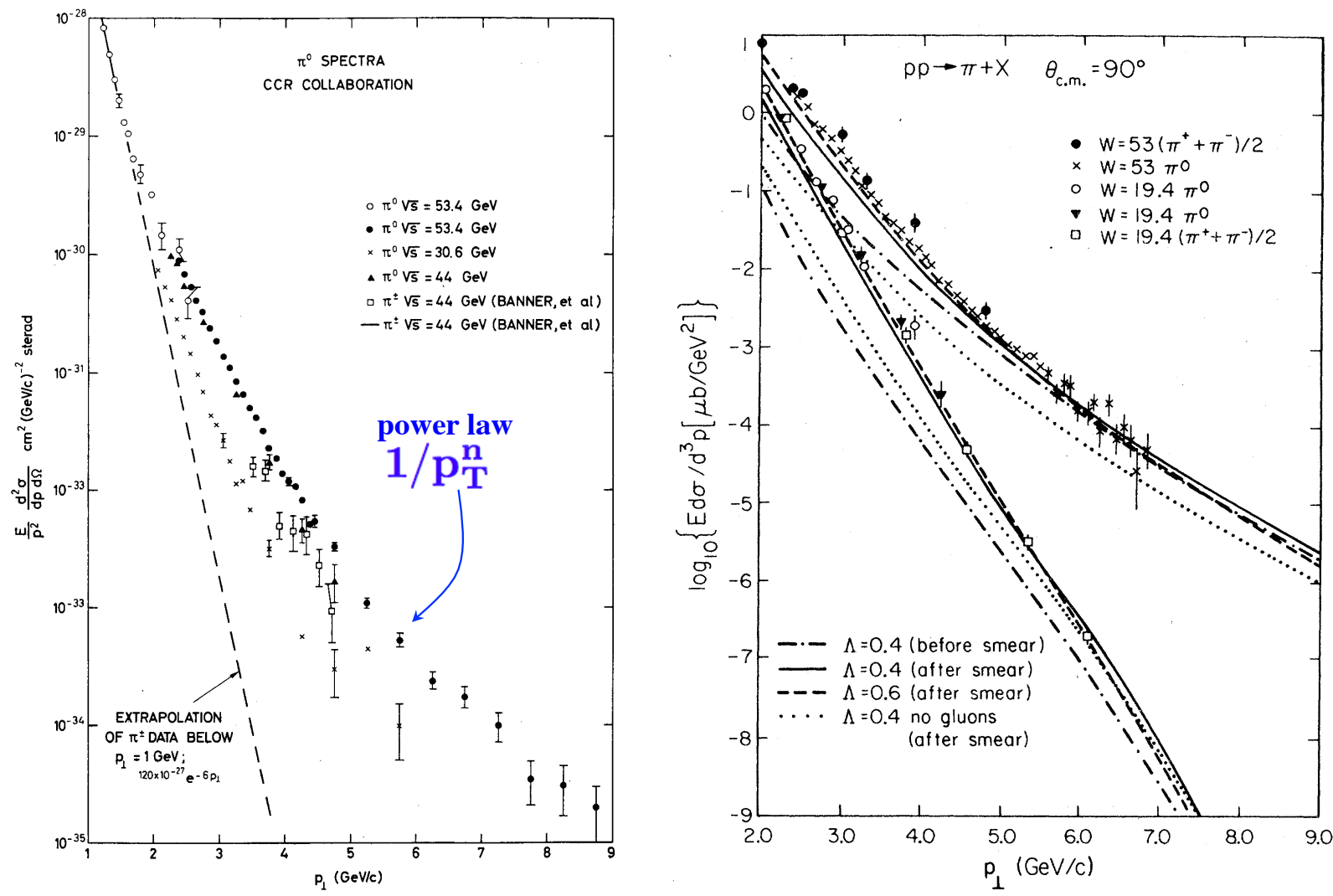

Figure 7: a)(left) Plot of invariant single $\pi^{0}$ cross section vs. $p_{T}$ for several $\sqrt{s}$ from CCR at the CERN-ISR [33]. b)(right) Feynman, Field, and Fox [35] QCD calculation of mid-rapidity high- $p_{T}$ $\pi$ spectra at $\sqrt{s}=19.4$ and $53 \mathrm{GeV}$, with and without $k_{T}$ smearing, for two values of $\Lambda_{\mathrm{QCD}}$.

agreement with the high $p_{T}$ single particle spectra were published in 1978, most experimentalists in the U. S., notably at the first Snowmass conference in July 1982, were skeptical because of evidence against jets presented at the ICHEP1980 by a CERN experiment, NA5 [36].

Bjorken had proposed in 1973 [37] that jets from the fragmentation of high $p_{T}$ scattered partons should be observed using " $4 \pi$ " hadron calorimeters. The first large aperture measurement was by 

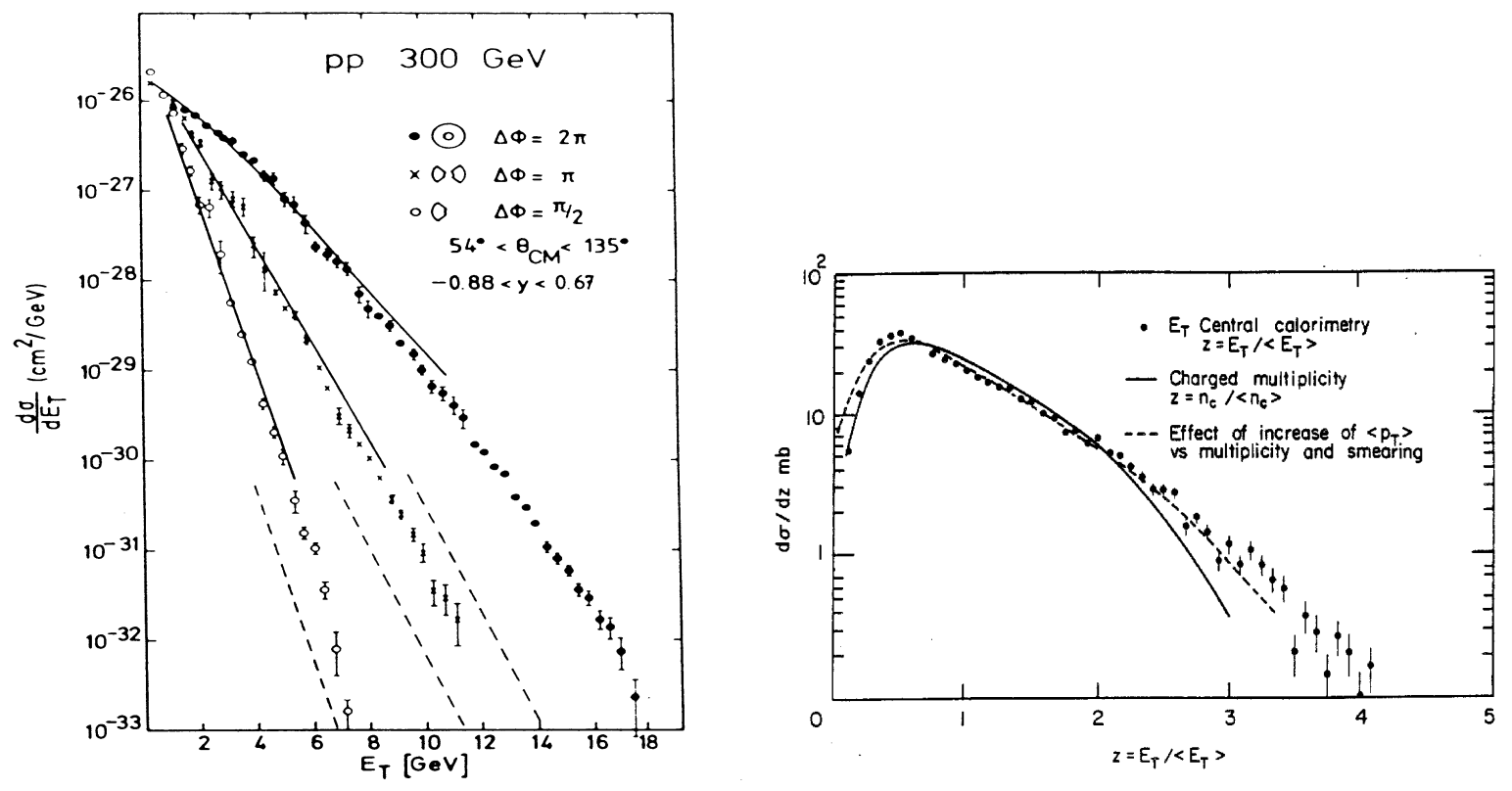

Figure 8: a)(left) $E_{T}$ distributions [36] in the solid angles indicated. Predictions from soft (low $p_{T}$ ) multiparticle production and QCD hard-scattering are shown by solid and dashed curves respectively. b) (right) $E_{T}$ distribution [39] for $|\eta| \leq 3$ from $\overline{\mathrm{p}}$-p collisions at $\sqrt{s}=540 \mathrm{GeV}$ in the KNO type variable, $z=E_{T} /\left\langle E_{T}\right\rangle$, used for multiplicity [41].

NA5 [36] at the CERN-SpS (Fig. 83) who showed a transverse energy $\left(E_{T}\right)$ spectrum at ICHEP1980, where the sum:

$$
E_{T}=\sum_{i} E_{i} \sin \theta_{i}
$$

is taken over all particles emitted into a fixed solid angle for each event. In Fig. 8a [36], the solid angle varies from full azimuthal acceptance, $\Delta \phi=2 \pi$, in the c.m. rapidity range $-0.88<y<0.76$, to smaller azimuthal regions as shown on the figure. The striking results, which contradicted a previous claim from Fermilab [38], were: i) no jets were seen in the full azimuth data; ii) all the data were far above the QCD predictions; iii) the large $E_{T}$ observed was the result of "a large number of particles with a rather small transverse momentum".

As we shall see below, $E_{T}$ distributions are very important in Relativistic Heavy Ion (RHI) physics since they can be used to characterize and study the nuclear geometry of an $\mathrm{A}+\mathrm{B}$ reaction on an event-by-event basis. The strong relation between $E_{T}$ and multiplicity distributions and the absence of jets in these distributions was emphasized in a talk by UA1 at ICHEP 1982 (Fig. 8b) [39]. Ironically, this talk immediately followed a talk by UA2 [40] which provided the first evidence for a di-jet from hard-scattering at a level 5-6 orders of magnitude down in the $E_{T}$ distribution from $\overline{\mathrm{p}}$-p collisions at $\sqrt{s}=540 \mathrm{GeV}$.

There are many additional important results in high-energy physics from this period that are relevant to both QCD and RHI physics, which must be skipped in this brief introduction. I have covered some of these results in previous ISSP lectures and proceedings [12, 13]; but I wrote a book with Jan Rak [14, which was published in mid-2013, that covers this information in detail and which is the real introduction to what follows. 


\section{Introduction to QGP Physics}

Given that I already said that the QGP was discovered at RHIC, what further studies are important? The QGP is the only place in the universe where we can in principle and in practice study Quantum Chromo-Dynamics (QCD) for color-charged quarks in a color-charged medium. For in-

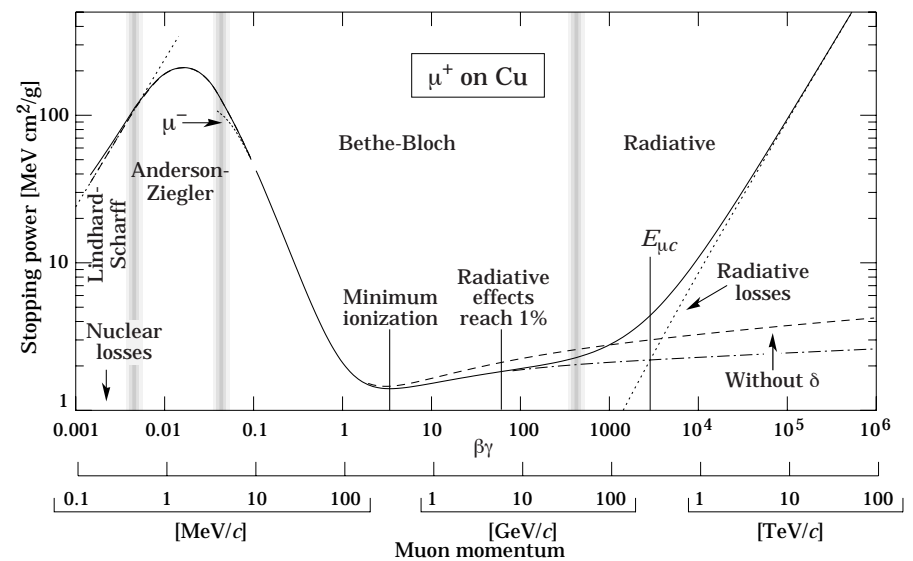

Figure 9: $d E / d x$ of a $\mu^{+}$in Copper as a function of muon momentum [42].

stance, how long will it take before we understand the passage of a quark through a QGP medium as well as we understand the passage of a muon through Copper in QED (Fig. 9) [42.

Of course, in addition to understanding the behavior of QCD in a medium, the central goal of our field is a quantitative study of the phases of nuclear matter. This requires a broad, quantitative study of the fundamental properties of the QGP including the extraction of the transport coefficients of the medium such as critical temperature, $T_{c}$, speed of sound, $c_{s}$, the ratio of shear viscosity to entropy density, $\eta / s$, etc. To help understand how we shall proceed to address these issues, it is important to understand how we got to this point.

The year 2013 was the 13th year of RHIC operation, and the two major detectors PHENIX and STAR which study the QGP at RHIC are basically first round detectors with a few incremental upgrades. Thus, the design of these detectors was heavily influenced by the c. 1990 view of the signatures of the QGP, which as noted above were quite different from what was discovered.

\section{$4.1 J / \Psi$ suppression - the original "gold-plated" QGP signature}

Since 1986, the 'gold-plated' signature of deconfinement was thought to be $J / \Psi$ suppression. Matsui and Satz [43] proposed that $J / \Psi$ production in $\mathrm{A}+\mathrm{A}$ collisions would be suppressed by Debye screening of the quark color charge in the QGP. The $J / \Psi$ is produced when two gluons interact to produce a $c, \bar{c}$ pair which then resonates to form the $J / \Psi$. In the plasma the $c, \bar{c}$ interaction is screened so that the $c, \bar{c}$ go their separate ways and eventually pick up other quarks at the periphery to become open charm.

"Anomalous suppression" of $J / \Psi$ was found in $\sqrt{s_{N N}}=17.2 \mathrm{GeV} \mathrm{Pb}+\mathrm{Pb}$ collisions at the CERN-SpS [44] (Fig. 10 a). This is the CERN fixed target heavy ion program's main claim to fame: but the situation has always been complicated because the $J / \Psi$ is suppressed in p+A collisions. For example, in $\sqrt{s_{N N}}=38.8 \mathrm{GeV} \mathrm{p}+\mathrm{A}$ collisions [45] (Fig. 10b) the Drell-Yan $\bar{q} q \rightarrow \mu^{+} \mu^{-}$crosssection per nucleon is constant as a function of mass number, A, which indicates the expected absence of shadowing in a nucleus for point-like production processes; while the $J / \Psi$ and $\Upsilon$ cross sections per nucleon are suppressed by an amount $A^{\alpha}$ with $\alpha=0.920 \pm 0.008$ for both $J / \Psi$ and $\Psi^{\prime}$ and $\alpha=0.96 \pm 0.01$ for both the $\Upsilon_{1 s}$ and $\Upsilon_{2 s+3 s}$. This is called a Cold Nuclear Matter or CNM 

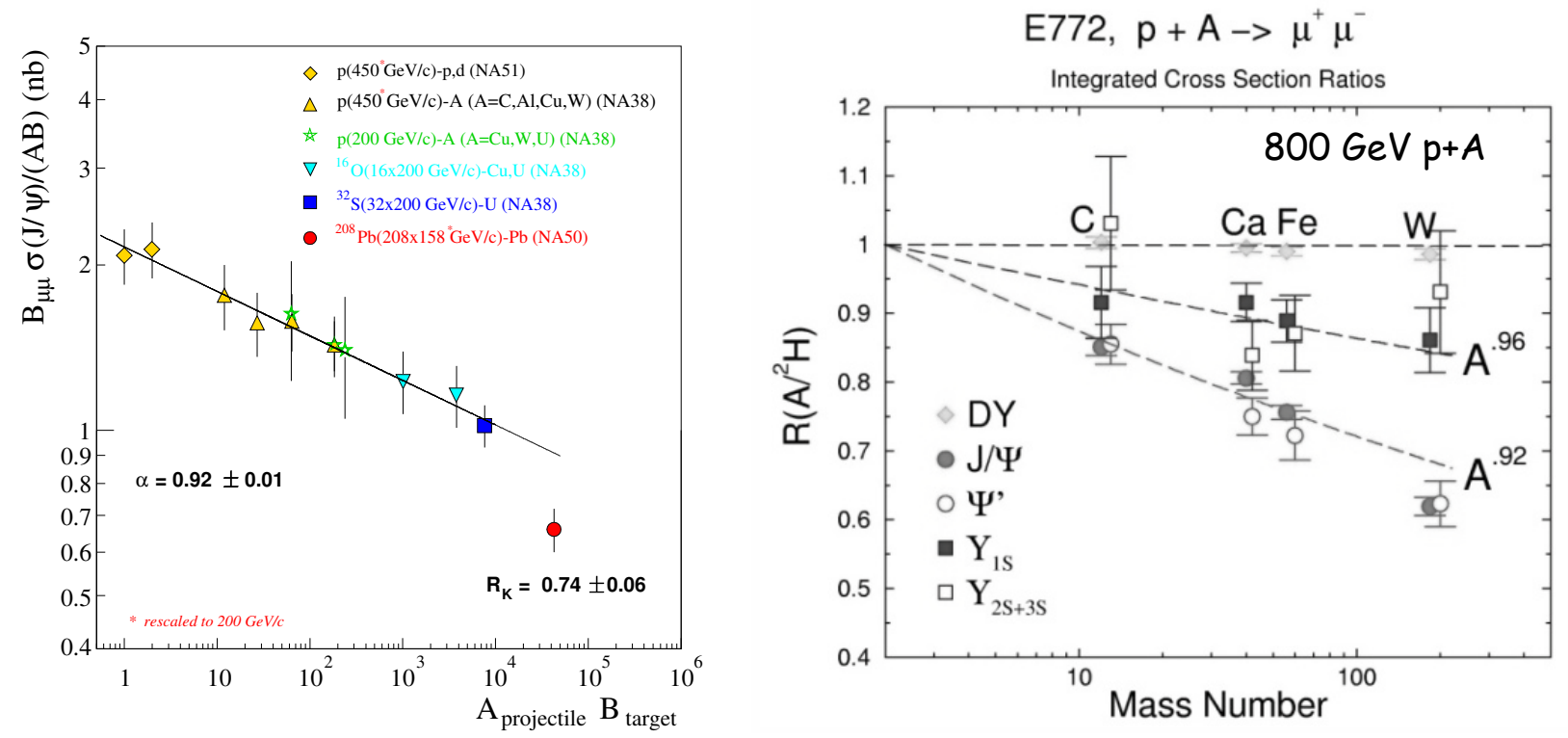

Figure 10: a) (left) Total cross section for $J / \Psi$ production divided by $A B$ in $\mathrm{A}+\mathrm{B}$ collisions at $158-200 A$ $\mathrm{GeV}$ [4] (note the ${ }^{*}$ ). b) (right) $A$ dependence of charmonium and Drell-Yan pair production in $800 \mathrm{GeV}$ $\mathrm{p}+\mathrm{A}$ collisions [45] expressed as the ratio of heavy nucleus to deuterium cross sections per nucleon. The dashed lines are fits to $A^{\alpha}$ for the CNM effect, with the values of $\alpha$ indicated.

effect and is shown as the line with $\alpha=0.92$ on Fig. $10 \mathrm{a}$. The "Anomalous suppression" is the difference between the data point at $A B=208 \times 208$ and the line, provided that the CNM effect is the same at $\sqrt{s_{N N}}=17.2$ and $38.8 \mathrm{GeV}$.

The later development of $J / \Psi$ measurements, after it was shown that $J / \Psi$ suppression was the same at RHIC at $\sqrt{s_{N N}}=200 \mathrm{GeV}$ as at the CERN-SpS (e.g. see Ref. [13]), has not been concerned with $J / \Psi$ suppression as a signature of deconfinement, but rather with the strong c.m. energy dependence of the CNM effect and the possibility of regeneration of $J / \Psi$ from recombination of the large number of $c$ and $\bar{c}$ quarks produced in the QGP. Nevertheless, the search for $J / \Psi$ suppression (as well as thermal photon/dilepton radiation from the QGP) drove the design of the RHIC experiments [17] and the ALICE experiment at the LHC [46]. Only recently have results from the ALICE experiment, to be discussed below, reopened the issue of whether recombination implies deconfinement.

\subsection{Detector issues in $\mathrm{A}+\mathrm{A}$ compared to $\mathrm{p}-\mathrm{p}$ collisions}

A main concern of experimental design in RHI collisions is the huge multiplicity in $\mathrm{A}+\mathrm{A}$ central collisions compared to p-p collisions. A schematic drawing of a collision of two relativistic Au nuclei is shown in Fig. 11a. In the center of mass system of the nucleus-nucleus collision, the two Lorentz-contracted nuclei of radius $R$ approach each other with impact parameter $b$. In the region of overlap, the "participating" nucleons interact with each other, while in the non-overlap region, the "spectator" nucleons simply continue on their original trajectories and can be measured in Zero Degree Calorimeters (ZDC), so that the number of participants can be determined. The degree of overlap is called the centrality of the collision, with $b \sim 0$, being the most central and $b \sim 2 R$, the most peripheral. The maximum time of overlap is $\tau_{\circ}=2 R / \gamma c$ where $\gamma$ is the Lorentz factor and $c$ is the speed of light in vacuum.

The energy of the inelastic collision is predominantly dissipated by multiple particle production, 

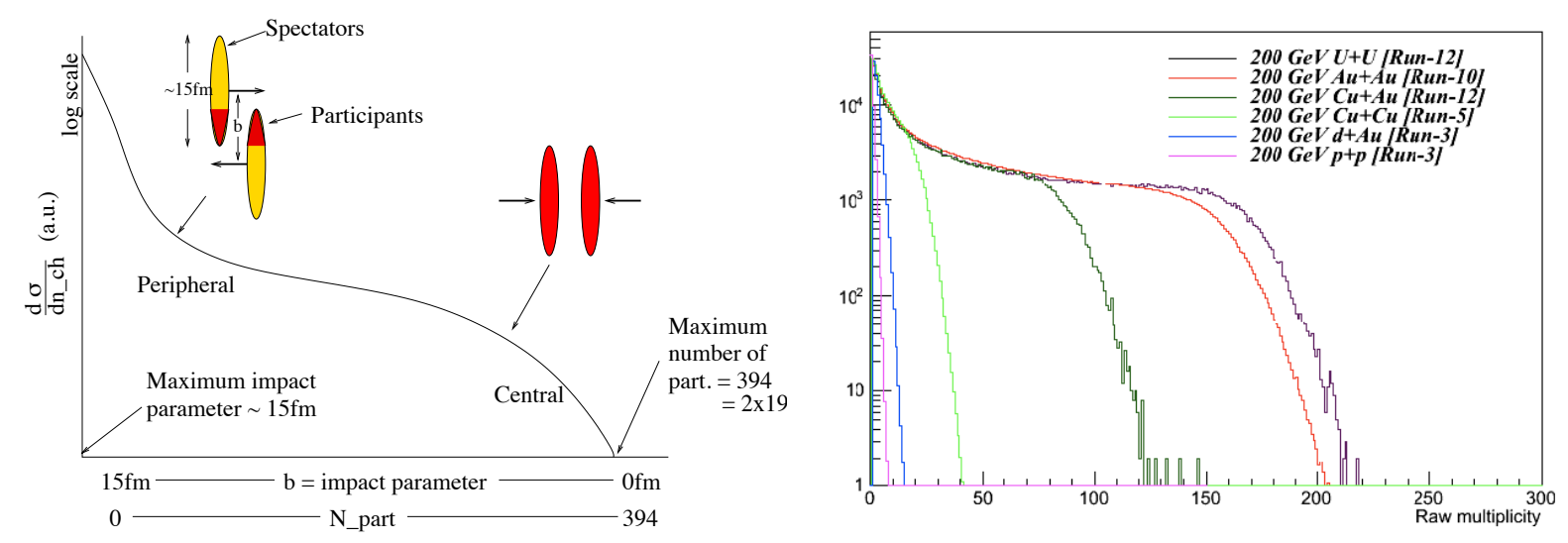

Figure 11: a) (left) Schematic of collision in the $N-N$ c.m. system of two Lorentz contracted nuclei with radius $R$ and impact parameter $b$. The curve with the ordinate labeled $d \sigma / d n_{\text {ch }}$ represents the relative probability of charged particle multiplicity $n_{\mathrm{ch}}$ which is directly proportional to the number of participating nucleons, $N_{\text {part }}$. b)(right) raw $n_{\text {ch }}$ distributions in p-p to U-U collisions at $\sqrt{s_{N N}}=200 \mathrm{GeV}$ from PHENIX [47].
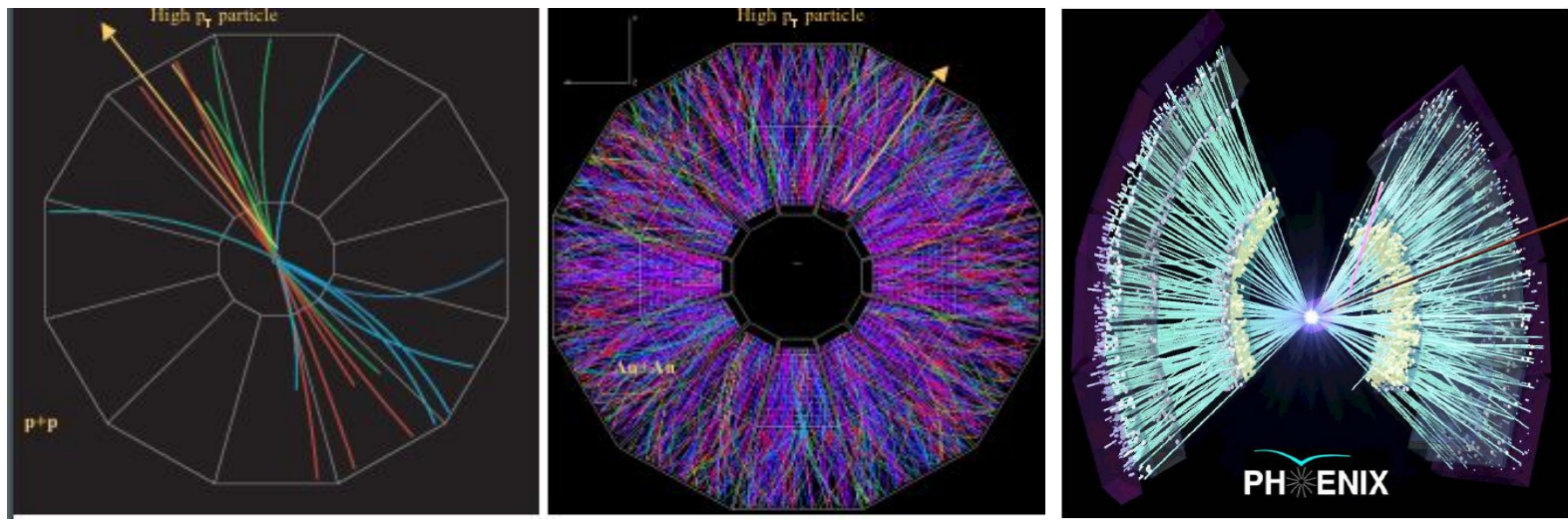

Figure 12: a) (left) A p-p collision in the STAR detector viewed along the collision axis; b) (center) $\mathrm{Au}+\mathrm{Au}$ central collision at $\sqrt{s_{N N}}=200 \mathrm{GeV}$ in STAR; c) (right) Au+Au central collision at $\sqrt{s_{N N}}=200 \mathrm{GeV}$ in PHENIX.

where $N_{\mathrm{ch}}$, the number of charged particles produced, is directly proportional [8] to the number of participating nucleons $\left(N_{\text {part }}\right)$ as sketched on Fig. 11 a. Thus, $N_{\text {ch }}$ or the total transverse energy $E_{T}$ in central $\mathrm{Au}+\mathrm{Au}$ collisions is roughly $A$ times larger than in a p-p collision, as shown in actual events from the STAR and PHENIX detectors at RHIC (Fig. 12). Figure 11b shows the measured distributions [47] of the charged particle multiplicity, $n_{\mathrm{ch}}$, at mid-rapidity at $\sqrt{s_{N N}}=200 \mathrm{GeV}$ for all the combinations of $\mathrm{A}+\mathrm{B}$ collisions measured at RHIC. The increase of $n_{\mathrm{ch}}$ with both $A$ and $B$ is evident. The impact parameter $b$ can not be measured directly, so the centrality of a collision is defined in terms of the upper percentile of $n_{\mathrm{ch}}$ or $E_{T}$ distributions, e.g. top 10\%-ile, upper $10-20 \%$-ile. Unfortunately the "upper" and "-ile" are usually not mentioned which sometimes confuses the uninitiated.

In Fig. 13, measurements of the charged particle multiplicity density $d N_{\mathrm{ch}} / d \eta$ at mid-rapidity, $|\eta|<0.5$, relative to the number of participating nucleons, $N_{\text {part }}$, are shown as a function of central- 


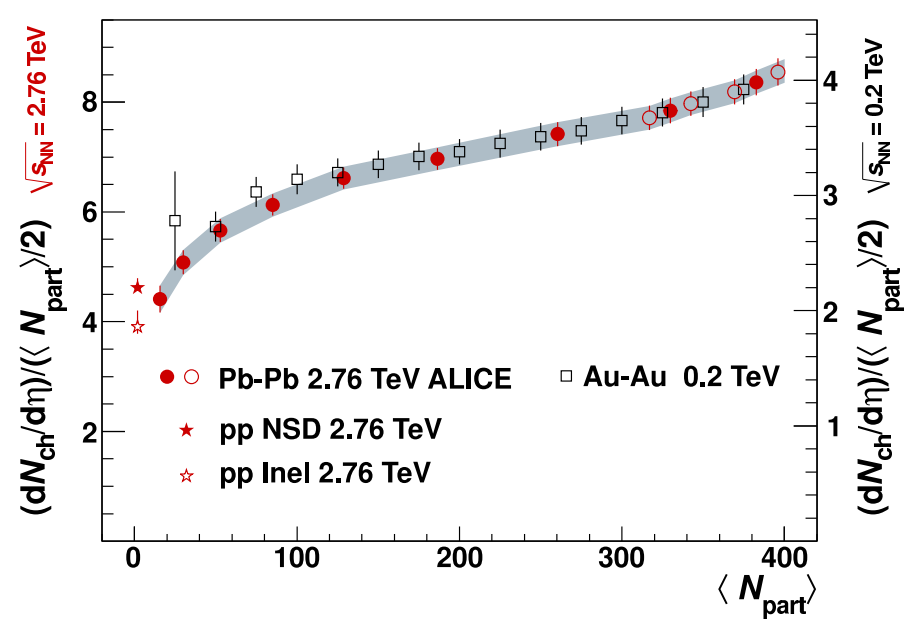

Figure 13: Dependence of $\left(d N_{\mathrm{ch}} / d \eta\right) /\left(N_{\text {part }} / 2\right)$ on the average number of participants $\left\langle N_{\text {part }}\right\rangle$ in bins of centrality, for $\mathrm{Pb}+\mathrm{Pb}$ collisions at $\sqrt{s_{N N}}=2.76 \mathrm{TeV}$ [49] and $\mathrm{Au}+\mathrm{Au}$ collisions at $\sqrt{s_{N N}}=0.200 \mathrm{TeV}$ [48]. The scale for the lower-energy data (right side) differs by a factor of 2.1 from the scale for the higher-energy data (left side).

ity for $\sqrt{s_{N N}}=200 \mathrm{GeV} \mathrm{Au}+\mathrm{Au}$ collisions at RHIC [48] together with new results from ALICE in $\sqrt{s_{N N}}=2.76 \mathrm{TeV} \mathrm{Pb}+\mathrm{Pb}$ collisions at LHC [49]. The results are expressed as $\left(d N_{\mathrm{ch}} / d \eta\right) /\left(N_{\text {part }} / 2\right)$ for easy comparison to p-p collisions.

The LHC data show the effect well known from RHIC that $d N_{\mathrm{ch}} / d \eta$ does not depend linearly on $N_{\text {part }}$, since $\left(d N_{\mathrm{ch}} / d \eta\right) /\left(N_{\text {part }} / 2\right)$ is not a constant for all $N_{\text {part }}$. However the data also show the amazing effect that the ratio of $\left(d N_{\mathrm{ch}} / d \eta\right) /\left(N_{\text {part }} / 2\right)$ from LHC to RHIC is simply a factor of 2.1 in every centrality bin. Thus the LHC and RHIC data lie one on top of each other by simple scaling of the RHIC measurements by a factor of 2.1. This is an incredibly beautiful result which shows that in going from $\mathrm{p}-\mathrm{p}$ to $\mathrm{A}+\mathrm{A}$ collisions, the charged particle production is totally dominated by the nuclear geometry of the $\mathrm{A}+\mathrm{A}$ collisions represented by the number of participating nucleons, $N_{\text {part }}$, independently of the nucleon-nucleon c.m. energy, $\sqrt{s_{N N}}$.

Since it is a huge task to reconstruct the momenta and identity of all the particles produced in these events, the initial detectors at RHIC [17] concentrated on the measurement of singleparticle or multi-particle inclusive variables to analyze RHI collisions, with inspiration from the CERN ISR which emphasized those techniques before the era of jet reconstruction [50]. There are two major detectors in operation at RHIC, STAR and PHENIX, and there were also two smaller detectors, BRAHMS and PHOBOS, which have completed their program. As may be surmised from Fig. 12, STAR, which emphasizes hadron physics, is most like a conventional general purpose collider detector, a TPC to detect all charged particles over the full azimuth $(\Delta \phi=2 \pi)$ and \pm 1 units of pseudo-rapidity $(\eta)$; while PHENIX is a very high granularity high resolution special purpose detector covering a smaller solid angle at mid-rapidity, together with a muon-detector at forward rapidity [51].

One nice feature of the STAR detector is the ability to measure the mass/charge of a particle from its momentum/charge and time of flight, and then use $d E / d x$ measured in the TPC to determine the charge. In this way STAR has observed many anti-nuclei, notably in 2011 the "Observation of the antimatter helium-4 nucleus" in Au+Au collisions [52] (Fig. 14 a). The differential invariant yields $d^{2} N /\left(2 \pi p_{T} d p_{T} d y\right)$ per central $\mathrm{Au}+\mathrm{Au}$ collision at $\sqrt{s_{N N}}=200 \mathrm{GeV}$ as a function of baryon number $\mathrm{B}$, evaluated at $p_{T} /|B|=0.875 \mathrm{GeV} / \mathrm{c}$, are shown in Fig. 14b and show a steady exponential decrease with increasing $B$ [53]. The anti-nuclei are made by coalescence of the large 

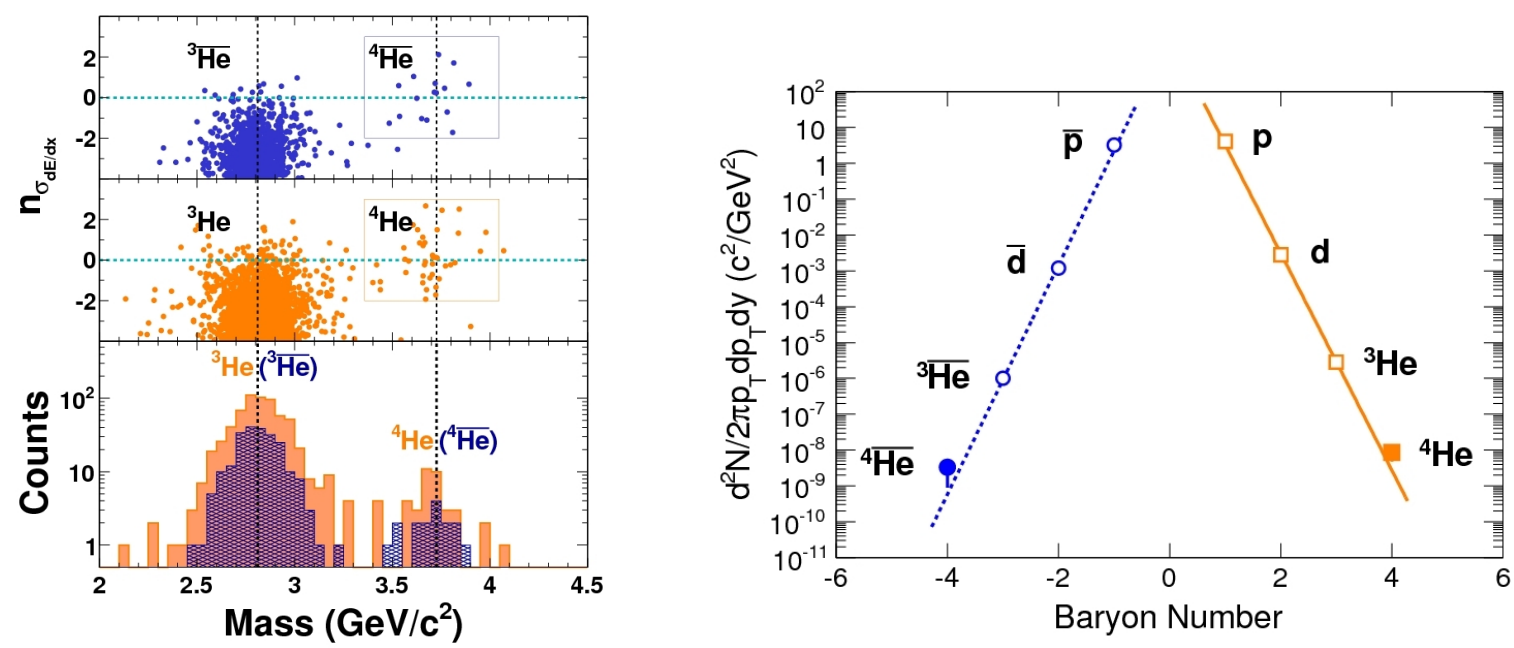

Figure 14: a) (left) Number of standard deviations, $n_{\sigma_{d E / d x}}$, of $d E / d x$ resolution from the expected value for ${ }^{4} \mathrm{He}$, for negative and positive particles as a function of calculated mass, and projected counts for $-2<n_{\sigma_{d E / d x}}<3$. b) Differential invariant yields as a function of baryon number, B.

number of $\bar{n}$ and $\bar{p}$ produced, an advantage of the high multiplicity.

PHENIX is designed to measure and trigger on rare processes involving leptons, photons and identified hadrons at the highest luminosities with the special features: i) a minimum of material $\left(0.4 \% X_{\circ}\right)$ in the aperture to avoid photon conversions; ii) possibility of zero magnetic field on axis to prevent de-correlation of $e^{+} e^{-}$pairs from photon conversions; iii) Electro-Magnetic Calorimeter (EMCal) and Ring Imaging Cherenkov Counter (RICH) for $e^{ \pm}$identification and level-1 $e^{ \pm}$trigger; iv) a finely segmented EMCal $(\delta \eta, \delta \phi=0.01 \times 0.01)$ to avoid overlapping showers due to the high multiplicity and for separation of single- $\gamma$ and $\pi^{0}$ up to $p_{T} \sim 25 \mathrm{GeV} / \mathrm{c}$; v) EMCal and precision Time of Flight measurement for particle identification. Some results uniquely possible with this detector such as measurements of direct photons via internal conversion to $e^{+} e^{-}$pairs will be discussed below.

In addition to the large multiplicity, there are two other issues in RHI physics which are different from p-p physics: i) space-time issues, both in momentum space and coordinate space - for instance what is the spatial extent of fragmentation? is there a formation time/distance?; ii) huge azimuthal anisotropies of particle production in non-central collisions (colloquially collective flow) which are very interesting in their own right and provide much richer features than originally envisaged.

\section{Collective Flow}

A distinguishing feature of $\mathrm{A}+\mathrm{A}$ collisions compared to either $\mathrm{p}-\mathrm{p}$ or $\mathrm{p}+\mathrm{A}$ collisions has been the collective flow observed. This effect is seen over the full range of energies studied in heavy ion collisions, from incident kinetic energy of $100 A \mathrm{MeV}$ to c.m. energy of $\sqrt{s_{N N}}=200 \mathrm{GeV}$ [54]. Collective flow, or simply flow, is a collective effect which can not be obtained from a superposition of independent $\mathrm{N}-\mathrm{N}$ collisions. Immediately after an $\mathrm{A}+\mathrm{A}$ collision, the overlap region defined by the nuclear geometry is almond shaped (see Fig 15 with the shortest axis along the impact parameter vector. Due to the reaction plane breaking the $\phi$ symmetry of the problem, the semiinclusive single particle spectrum is modified by an expansion in harmonics [57] of the azimuthal angle of the particle with respect to the reaction plane, $\phi-\Phi_{R}[58$, where the angle of the reaction 

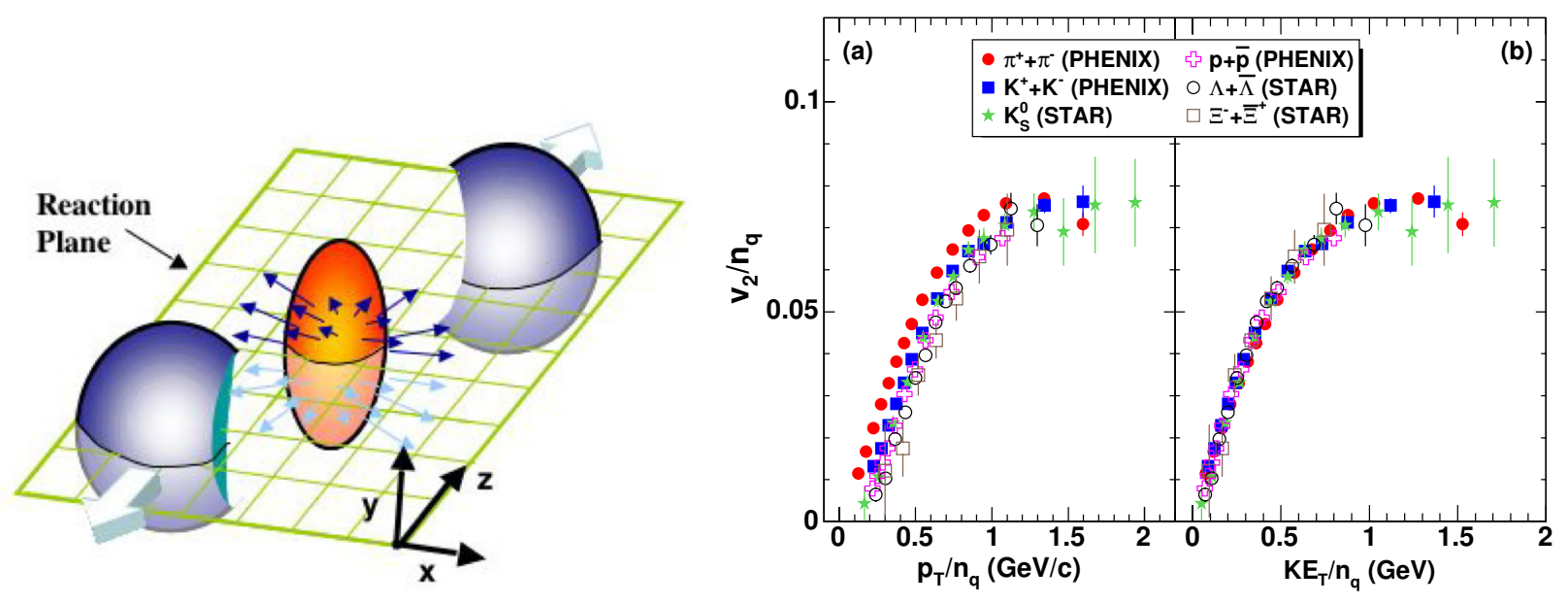

Figure 15: (left) Almond shaped overlap zone generated just after an $\mathrm{A}+\mathrm{A}$ collision where the incident nuclei are moving along the $\pm z$ axis. The reaction plane by definition contains the impact parameter vector (along the $x$ axis) [55]. (right) Measurements of elliptical-flow $\left(v_{2}\right)$ for identified hadrons plotted as $v_{2}$ divided by the number of constituent quarks $n_{q}$ in the hadron as a function of (a) $p_{T} / n_{q}$, (b) $K E_{T} / n_{q}[56$.

plane $\Phi_{R}$ is defined to be along the impact parameter vector, the $x$ axis in Fig. 15.

$$
\frac{E d^{3} N}{d p^{3}}=\frac{d^{3} N}{p_{T} d p_{T} d y d \phi}=\frac{d^{3} N}{2 \pi p_{T} d p_{T} d y}\left[1+\sum_{n} 2 v_{n} \cos n\left(\phi-\Phi_{R}\right)\right] .
$$

The expansion parameter $v_{2}$, called elliptical flow, is predominant at mid-rapidity. In general, the fact that flow is observed in final state hadrons shows that thermalization is rapid, so that hydrodynamics comes into play at a time, $\tau_{0}$, which is before the spatial anisotropy of the overlap almond dissipates. At this early stage hadrons have not formed and it has been proposed that the constituent quarks flow [59], so that the flow should be proportional to the number of constituent quarks $n_{q}$, in which case $v_{2} / n_{q}$ as a function of $p_{T} / n_{q}$ would represent the constituent quark flow as a function of constituent quark transverse momentum and would be universal. However, in relativistic hydrodynamics, at mid-rapidity, the transverse kinetic energy, $m_{T}-m_{0}=\left(\gamma_{T}-1\right) m_{0} \equiv K E_{T}$, rather than $p_{T}$, is the relevant variable; and in fact $v_{2} / n_{q}$ as a function of $K E_{T} / n_{q}$ seems to exhibit nearly perfect scaling [56] (Fig. $15 \mathrm{~b}$ ).

The fact that the flow persists for $p_{T}>1 \mathrm{GeV} / \mathrm{c}$ (Fig. 16]) implies that the viscosity is small [60], perhaps as small as a quantum viscosity bound from string theory [61], $\eta / s=1 /(4 \pi)$ where $\eta$ is the shear viscosity and $s$ the entropy density per unit volume. This has led to the description of the "sQGP" produced at RHIC as "the perfect fluid" [9]. An estimate [62] of $\eta / s$ for nuclear matter and for several common fluids, as a function of the fractional difference of the temperature from the critical temperature, at fixed pressure, is shown in Fig. 16 b. This particular estimate [62] for the QGP at RHIC is quite close to the quantum bound (solid line). Also, empirically, for all common fluids $\eta / s$ is a minimum at or near the critical point [63] which might suggest that the conditions at RHIC energies are near the QCD critical point.

\subsection{Two-Particle Correlations and Flow}

In addition to measuring flow by the correlation of individual particles to the reaction plane, it is also possible to measure flow by the correlation of two particles to each other. The advantage of 

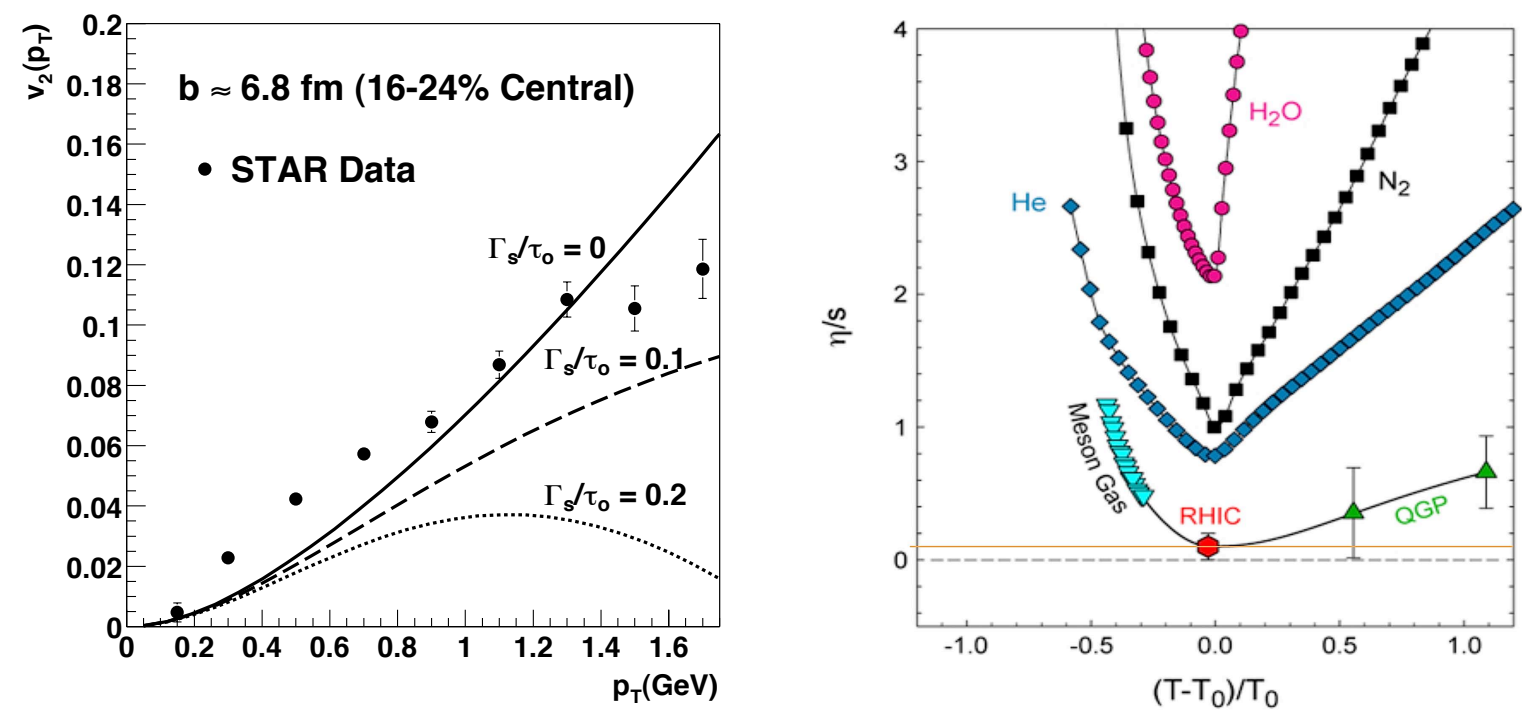

Figure 16: a) (left) Teaney's [60] predictions for $v_{2}\left(p_{T}\right)$ for ideal $\left(\Gamma_{s} / \tau_{0}=0\right)$ and viscous hydrodynamics, where $\Gamma_{s}=\frac{4}{3} \frac{\eta}{s T}$ is the sound attenuation length, and $\tau_{0}$ is the thermalization time. b) $\eta / s$ for various fluids at fixed pressure as a function of temperature $T$, where $T_{0}$ is the temperature of the critical point of the liquid-gas phase transition [62, 63].

this method is that one does not have to determine the reaction plane. Thus if two particles $A$ and $B$ are correlated to the reaction plane, but not otherwise correlated to each other,

$$
\frac{d N^{A}}{d \phi^{A}} \propto 1+\sum_{n} 2 v_{n}^{A} \cos \left(n\left(\phi^{A}-\Psi_{n}\right)\right), \quad \frac{d N^{B}}{d \phi^{B}} \propto 1+\sum_{n} 2 v_{n}^{B} \cos \left(n\left(\phi^{B}-\Psi_{n}\right)\right)
$$

then the correlation to the reaction plane induces a correlation of these two particles to each other which can be measured without knowledge of the reaction plane,

$$
\frac{d N^{A B}}{d \phi^{A} d \phi^{B}} \propto\left[1+2 v_{2}^{A} v_{2}^{B} \cos 2\left(\phi^{A}-\phi^{B}\right)+2 v_{3}^{A} v_{3}^{B} \cos 3\left(\phi^{A}-\phi^{B}\right)+\ldots\right] .
$$

In p-p collisions there is no collective flow but there are strong two-particle azimuthal correlations due to di-jet production in hard-scattering (see Fig. 12 a), which also exist in A+A collisions but are obscured by the large multiplicity [e.g. can you find a jet in Fig. 12 b]. Before the discovery of jets, two-particle correlations were used extensively at the CERN-ISR to study hard-scattering (the production of particles with large transverse momentum) in p-p collisions which was discovered there [50]. Due to the huge multiplicities in $\mathrm{Au}+\mathrm{Au}$ collisions at $\mathrm{RHIC}$, where for central $\mathrm{Au}+\mathrm{Au}$ collisions at $\sqrt{s_{N N}}=200 \mathrm{GeV}$, there is an estimated $\pi \Delta r^{2} \times \frac{1}{2 \pi} \frac{d E_{T}}{d \eta} \sim 375 \mathrm{GeV}$ of energy in one unit of the nominal jet-finding cone, $\Delta r=\sqrt{(\Delta \eta)^{2}+(\Delta \phi)^{2}}$, two-particle correlations were used exclusively for the first 10 years to study hard scattering at RHIC.

Typical examples of the di-hadron measurements in p-p and Au+Au central (0-20\%) collisions at $\sqrt{s_{N N}}=200 \mathrm{GeV}$ are shown in Fig. 17] 64, 65] which are presented as azimuthal distributions of the conditional yields of associated particles, with $p_{T_{a}}$, with respect to trigger particles with $3 \leq p_{T_{t}} \leq 10 \mathrm{GeV} / \mathrm{c}$. The di-jet structure in p-p collisions is clearly indicated by the gaussian-like strong azimuthal correlation peaks on the same side $\left(\Delta \phi=\phi_{a}-\phi_{t} \sim 0\right)$ and away side $(\Delta \phi \sim \pi$ 

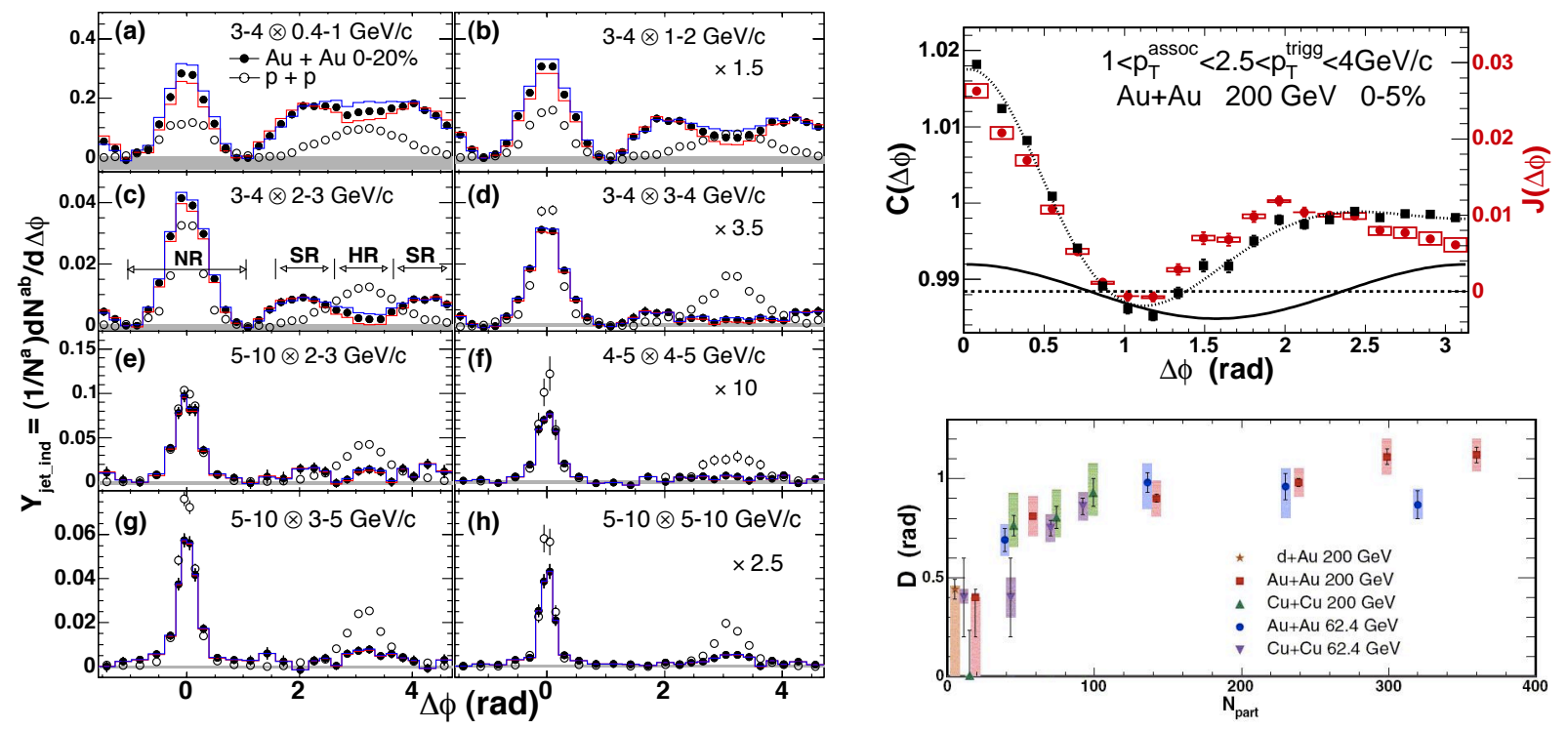

Figure 17: a-h) (left) PHENIX [64] azimuthal correlation conditional yield of associated $h^{ \pm}$particles with $p_{T_{a}}$ for trigger $h^{ \pm}$with $p_{T_{t}}$ for the various $p_{T_{t}} \otimes p_{T_{a}}$ combinations shown. i) (right)-(top) PHENIX [65] azimuthal correlation function $C(\Delta \phi)$ of $h^{ \pm}$with $1 \leq p_{T_{a}} \leq 2.5 \mathrm{GeV} / \mathrm{c}$ with respect to a trigger $h^{ \pm}$with $2.5 \leq p_{T_{t}} \leq 4 \mathrm{GeV} / \mathrm{c}$ in $\mathrm{Au}+\mathrm{Au}$ central collisions, where the line with data points indicates $C(\Delta \phi)$ before correction for the azimuthally modulated $\left(v_{2}\right)$ background, and the other line is the $v_{2}$ correction which is subtracted to give the jet correlation function $J(\Delta \phi)$ (data points). j) (right)-(bottom) PHENIX $D$ parameters [65], the angular distance of the apparently displaced peak of the $J(\Delta \phi)$ distribution from the angle $\Delta \phi=\pi$ as a function of centrality, represented as the number of participants $N_{\text {part }}$, for the systems and c.m. energies indicated.

rad.) relative to the trigger particle for all ranges of $p_{T_{t}}$ and $p_{T_{a}}$ measured. However, one of the many interesting features in $\mathrm{Au}+\mathrm{Au}$ collisions is that the away side azimuthal jet-like correlations (Fig. 17k) are much wider than in p-p collisions and show a two-lobed structure ("the shoulder" (SR)) at lower $p_{T_{t}}$ with a dip at $180^{\circ}$, reverting to the more conventional structure of a peak at $180^{\circ}$ ("the head" (HR)) for larger $p_{T_{t}}$.

The wide away-side correlation in central $\mathrm{Au}+\mathrm{Au}$ collisions is significantly obscured by the large multiparticle background which is modulated in azimuth by the $v_{2}$ collective flow of a comparable width to the jet correlation (Fig. 17 ). After the $v_{2}$ correction, the double peak structure $\sim \pm 1$ radian from $\pi$, with a dip at $\pi$ radians, becomes evident. The double-peak structure may indicate a reaction of the medium to a passing parton in analogy to a "sonic boom" or the wake of a boat, which was given the name "Mach Cone" [66], and has been under active study both theoretically [67] and experimentally. PHENIX characterizes this effect by the half-width $D(\sim 1.1$ radian $)$ of the Jet function, $J(\Delta \phi)$, the angular distance of the displaced peak of the distribution from the angle $\Delta \phi=\pi$. One of the striking features of the wide away side correlation is that the width $D$ (Fig. 17.j) does not depend on centrality, angle to the reaction plane, $p_{T_{a}}$ and $\sqrt{s_{N N}}$, which always seemed problematic to me if the effect were due to a reaction to the medium. Another suspicious issue is that the same effect occurs even for auto-correlations of particles with very low $p_{T}$ between 0.2 and $0.4 \mathrm{GeV} / \mathrm{c}$ where any effect of hard-scattered partons should be submerged by the predominant soft physics (Fig. 18a) 68.

In addition to the Head/Shoulder or Mach Cone effect in two-particle correlations on the awayside, same-side correlations also show a new effect in A+A collisions called "the Ridge" 69. This 

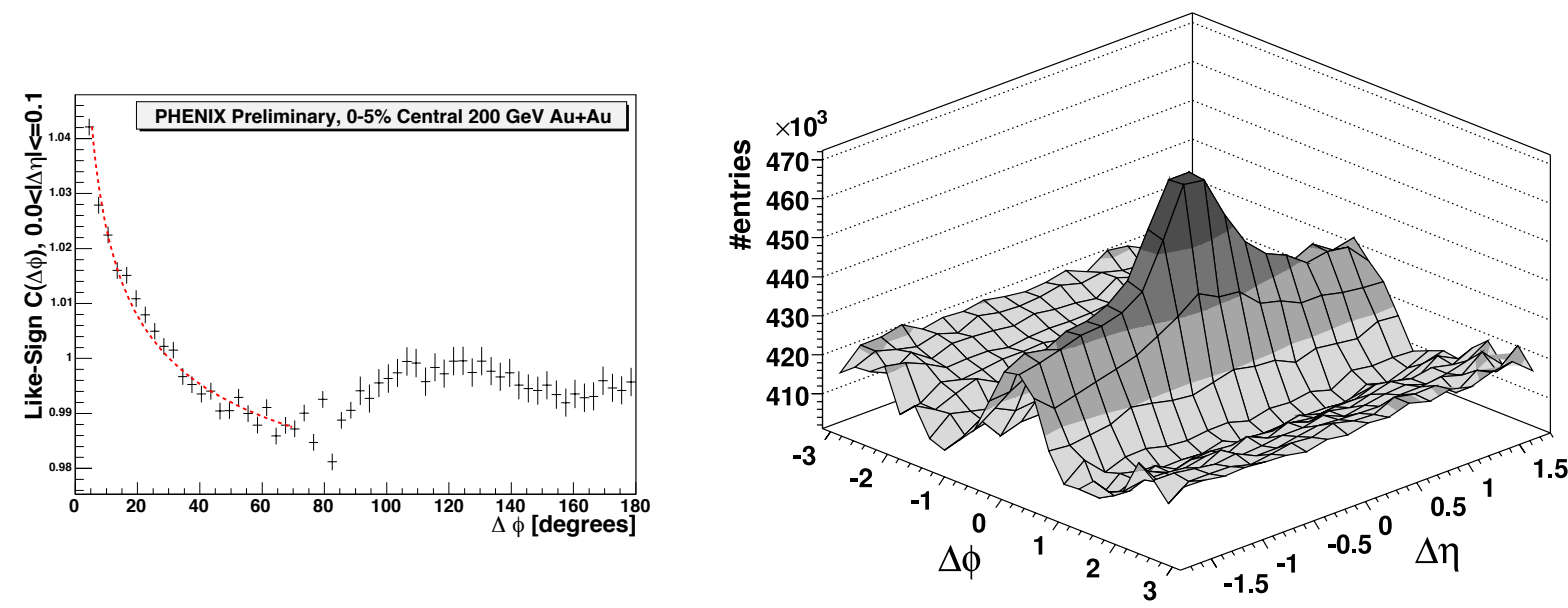

Figure 18: a) (left) Low $p_{T}$ like-sign pair azimuthal correlation function for $0-5 \%$ central $\mathrm{Au}+\mathrm{Au}$ collisions at $\sqrt{s_{N N}}=200 \mathrm{GeV}$ from charged hadrons with $0.2 \leq p_{T_{1}}, p_{T_{2}} \leq 0.4 \mathrm{GeV} / \mathrm{c}$ [68]. b) (right) "The Ridge" [70, 71].

is seen in two-dimensional correlations in $\Delta \eta, \Delta \phi$ (Fig. 18b) [70, 71] where the associated yield distribution can be decomposed into a narrow jet-like peak at small angular separation which has a similar shape to that found in p-p collisions, and a component that is narrow in $\Delta \phi$ but depends only weakly on $\Delta \eta$, the "ridge." However, new results in 2010-2011 dramatically changed this picture.

\subsection{Triangular flow, odd harmonics}

For the first 10 years of RHIC running, and dating back to the Bevalac, all the experts thought that the odd harmonics in Eq. 2 would vanish by the symmetry $\phi \rightarrow \phi+\pi$ of the almond shaped overlap region [72] (Fig. 15). However, in the year 2010, an MIT graduate student an his Professor in experimental physics, seeking (at least since 2006) how to measure the fluctuations of $v_{2}$ in the PHOBOS experiment at RHIC, realized that fluctuations in the collision geometry on an event-byevent basis, i.e. the distribution of participants from event-to-event, did not respect the average symmetry. This resulted in what they called "participant triangularity" and "triangular flow", or $v_{3}$ in Eq. 2, which they measured using both PHOBOS and STAR data [73] 2

Many experiments presented measurements of $v_{3}$ at Quark Matter 2011, e.g. Fig. 19 [75], and it was one of the most exciting results of that year. There are two striking observations from Fig. 19 which indicate that fluctuations of the initial collision geometry are driving the observed $v_{3}$ : i) the centrality dependence of $v_{3}\left(p_{T}\right)$ is weak as one would expect from fluctuations, but $v_{2}\left(p_{T}\right)$ which is most sensitive to the geometry of the "almond"-shaped overlap region tracks the change in eccentricity with centrality; ii) for the most central collisions $(0-10 \%)$, where the overlap region is nearly circular so that all the $v_{n}$ are driven by fluctuations, $v_{2}\left(p_{T}\right), v_{3}\left(p_{T}\right), v_{4}\left(p_{T}\right)$ are comparable. The fact that the observed collective flow of final state particles follows the fluctuations in the initial state geometry points to real hydrodynamic flow of a nearly perfect fluid (and convinces this author of the validity of hydrodynamics in RHI collisions, of which he was quite skeptical previously [13]).

\footnotetext{
${ }^{2}$ It was pointed out by Leticia Palhares in the discussion that a Brazilian group showed in 2009 that that the ridge and the cone, i.e. $v_{3}$, does appear in an event-by-event hydrodynamics calculation without jets [74], but the MIT group [73] was the first to show it with real data.
} 


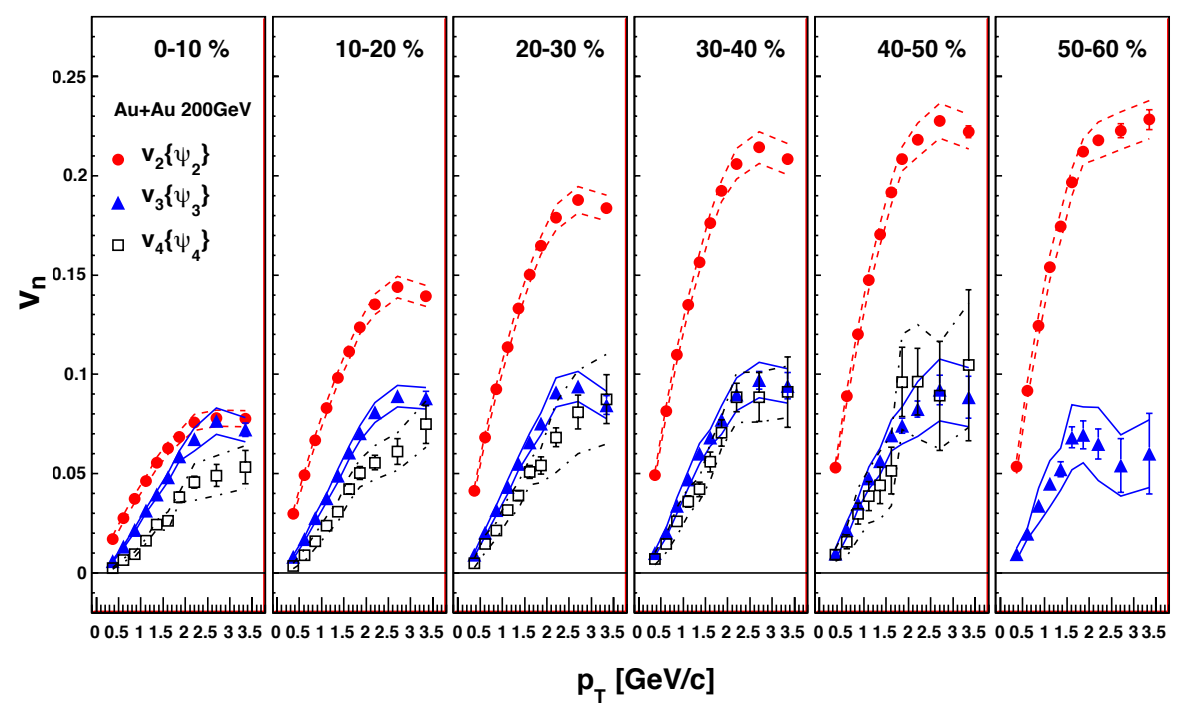

Figure 19: PHENIX [75] measurements of the $v_{n}$ parameters using Eq. 2 (with the appropriate reaction plane) as a function of $p_{T}$ for different centrality slices in $\sqrt{s_{N N}}=200 \mathrm{GeV} \mathrm{Au}+\mathrm{Au}$ collisions.

It is evident that $v_{3}$, a $\cos 3(\Delta \phi)$ term with lobes at $\Delta \phi=0,2 \pi / 3$ and $4 \pi / 3 \approx 0,2,4$ radians, would explain the peaks at $\pi \pm D$ radian in the two-particle correlations (Fig. 17,, $\mathrm{j}$ ) and also why $D \approx 1$ radian independent of centrality and kinematic variables; while the lobe at $\Delta \phi=0$ explains the ridge (Fig. 18b). There is presently lots of activity to confirm in detail whether taking account of the odd harmonics in addition to $v_{2}$ and $v_{4}$ in the background of Fig. 17. will result in gaussian-like away-jet peaks in $\mathrm{A}+\mathrm{A}$ collisions and the disappearance of the same-side ridge.

\section{RHIC beam energy scan-In search of the critical point}

In the years 2010-2011, RHIC made runs with $\mathrm{Au}+\mathrm{Au}$ collisions at c.m. energies $\sqrt{s_{N N}}=7.7,11.5$, $39,19.6,62.4 \mathrm{GeV}$, in addition to previous runs at 130 and $200 \mathrm{GeV}$, to search for the onset of large fluctuations which should occur near a critical point in the phase diagram (Fig. 1). Such fluctuations in the $K / \pi$ and $K / p$ ratio had been claimed at the CERN SPS fixed target program near $\sqrt{s_{N N}}=8 \mathrm{GeV}$ and were presented [76] as "evidence of the onset of the deconfinement phase transition". At QM2011, STAR [77] presented many excellent results on this subject of which I show a small selection in Fig. 20.

Fig. 20 a shows the multiplicity distribution $d N_{\mathrm{ch}} / d \eta$ which maintains the characteristic Nuclear Geometry dominated shape (as in Fig. 11), stretching to higher multiplicity, $d N_{\mathrm{ch}} / d \eta$, with increasing $\sqrt{s_{N N}}$. Fig. $20 \mathrm{p}$ shows the $K^{+} / \pi^{+}$and $K^{-} / \pi^{-}$ratios over the entire range of $\sqrt{s_{N N}}$ measurements. The maximum of the $K^{+} / \pi^{+}$ratio near $\sqrt{s_{N N}}=8 \mathrm{GeV}$ is naturally explained [78] by the change in dominant $K^{+}$production from $K^{+} \Lambda$ to $K^{+} K^{-}$whose smooth increase with $\sqrt{s_{N N}}$ can be seen from the $K^{-} / \pi^{-}$ratio. The famous "horn", or apparent discontinuity, at $\sqrt{s_{N N}}=8$ $\mathrm{GeV}$ from the SPS data [76] is greatly smoothed when the new STAR data are added. Fig. 20; shows a smooth variation of the the STAR measurements of the fluctuations of the event-by-event $K / p$ ratio as a function of $\sqrt{s_{N N}}$, which differs dramatically from the huge effect claimed by the 

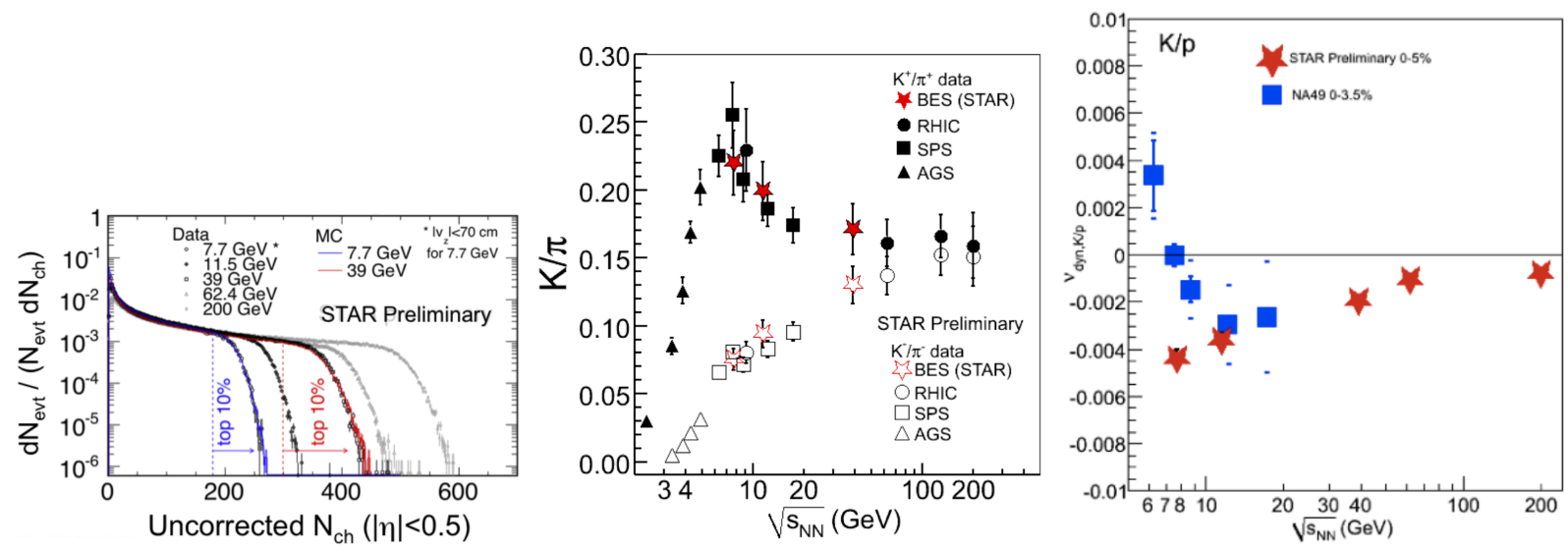

Figure 20: a) (left) $N_{\text {ch }}$ distribution in the STAR detector for 5 values of $\sqrt{s_{N N}}$ [77]; b) (center) $K / \pi$ ratio vs $\sqrt{s_{N N}}$ [77]; c) (right) Event-by-event fluctuation of $K / p$ ratio [77].

SPS Fixed Target measurements below $12 \mathrm{GeV}$, notably the change from negative to positive [76]. There is no doubt in this author's mind that one must prefer the collider measurements, where the detector position at mid-rapidity in the c.m. system is constant for all values of $\sqrt{s_{N N}}$, to the fixed target measurements, where the rapidity of the c.m. system moves dramatically with respect to the the detector as $\sqrt{s_{N N}}$ varies. This is a major strength of the RHIC Beam Energy Scan program.

\subsection{A press release during the 2011 school}

On June 23, 2011, shortly before I was to present my 2011 lectures, a press release from LBL arrived claiming that "By comparing theory with data from STAR, Berkeley Lab scientists and their colleagues map phase changes in the QGP" [79]. Since I criticized "physics by press-release" concerning the discovery of the QGP (above), I felt that I was obliged to review the physics behind this latest example, presumably a "Highlight from RHIC".

The subject is "Fluctuations of conserved quantities", in this case the net baryon distribution taken as $p-\bar{p}$. Since there can be no fluctuations of conserved quantities such as net charge or net baryon number in the full phase space, one has go to small intervals [80] to detect a small fraction of the protons and anti-protons which then fluctuates, i.e. varies from event to event. The argument is that, e.g. the fluctuation of one charged particle in or out of the considered interval produces a larger mean square fluctuation of the net electric charge if the system is in the hadron gas phase with integral charges than for the QGP phase with fractional charges.

However, while there are excellent statistical mechanical arguments about the utility of fluctuations of conserved quantities such as net baryon number as a probe of a critical point [81, there are, so far, no adequate treatments of the mathematical statistics of the experimental measurements. Theoretical analyses tend to be made in terms of a Taylor expansion of the free energy $F=-T \ln Z$ around the critical temperature $T_{c}$ where $Z$ is the partition function, or sum over states, which is of the form $Z \propto e^{-\left(E-\sum_{i} \mu_{i} Q_{i}\right) / k T}$ and $\mu_{i}$ are chemical potentials associated with conserved charges $Q_{i}$ [81]. The terms of the Taylor expansion, which are obtained by differentiation, are called susceptibilities, denoted $\chi$. The only connection of this method to mathematical statistics is that the Cumulant generating function in mathematical statistics is also a Taylor expansion of the ln of an exponential:

$$
g_{x}(t)=\ln \left\langle e^{t x}\right\rangle=\sum_{n=1}^{\infty} \kappa_{n} \frac{t^{n}}{n !} \quad \kappa_{m}=\left.\frac{d^{m} g_{x}(t)}{d t^{m}}\right|_{t=0} .
$$



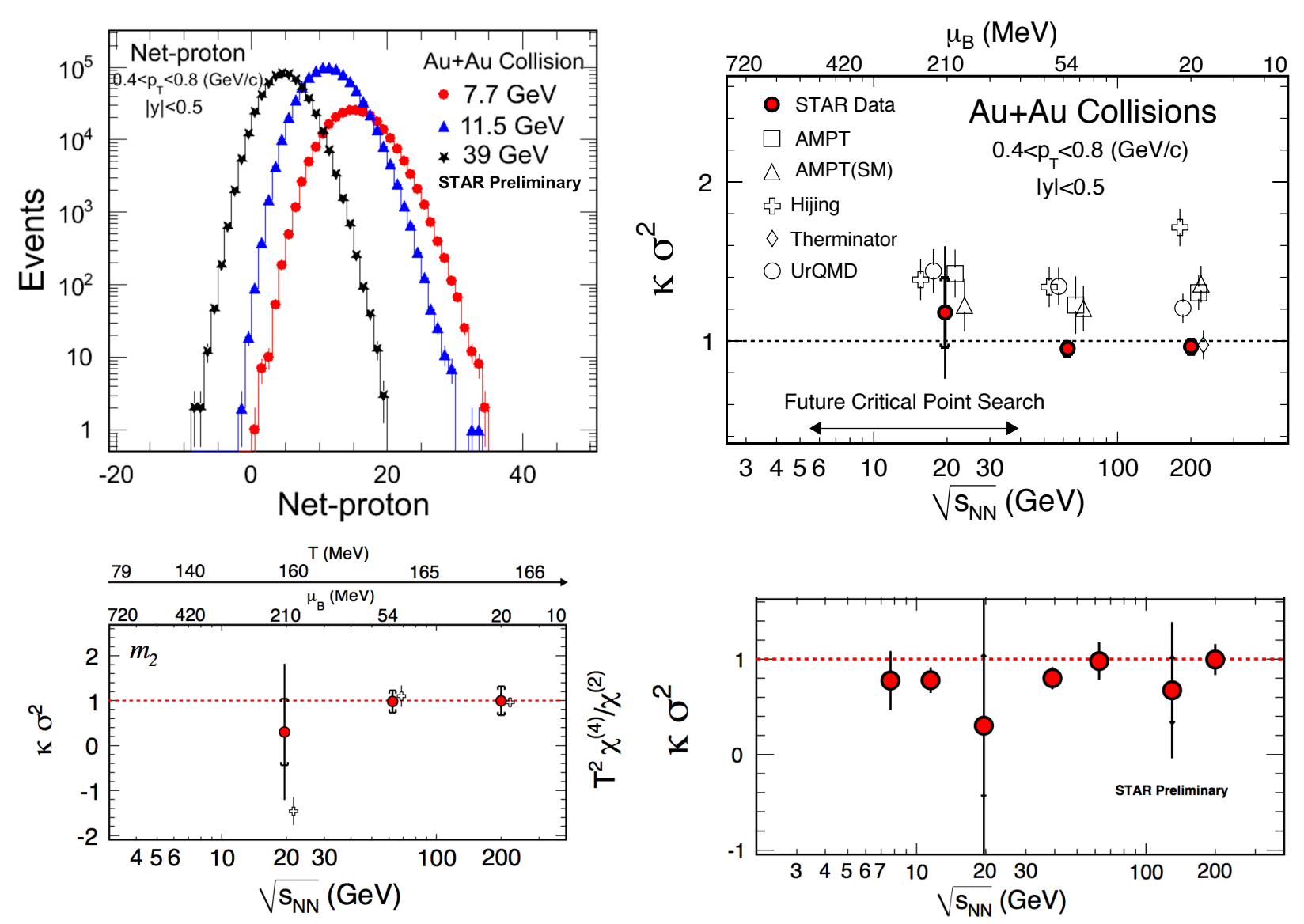

Figure 21: a) (top-left) STAR [83] distribution of event-by-event $p-\bar{p}$ at 3 values of $\sqrt{s_{N N}}$; b) (top-right) STAR published [84] measurements of $\kappa \sigma^{2}$; c) (bottom-left) Measurements from (b) as shown in Ref. 85. compared to the predicted ratio of susceptibilities (open crosses); d) (bottomright) compilation [83] of STAR measurements of $\kappa \sigma^{2}$.

Thus, the susceptibilities are Cumulants in mathematical statistics terms, where, in general, the Cumulant $\kappa_{m}$ represents the $m^{\text {th }}$ central moment with all $m$-fold combinations of the lower order moments subtracted ${ }^{3}$ For instance, $\kappa_{2}=\left\langle(x-\mu)^{2}\right\rangle \equiv \sigma^{2}, \kappa_{3}=\left\langle(x-\mu)^{3}\right\rangle, \kappa_{4}=\left\langle(x-\mu)^{4}\right\rangle-3 \kappa_{2}^{2}$, $\kappa_{5}=\left\langle(x-\mu)^{5}\right\rangle-10 \kappa_{3} \kappa_{2}$, where $\mu=\langle x\rangle$. Two so-called normalized or standardized Cumulants are common in this field, the skewness, $S=\kappa_{3} / \sigma^{3}$ and the kurtosis, $\kappa=\kappa_{4} / \sigma^{4}=\left\langle(x-\mu)^{4}\right\rangle / \sigma^{4}-3$.

A sample 83 of STAR measurements of the distribution of net-protons in $\mathrm{Au}+\mathrm{Au}$ collisions in the small interval $0.4 \leq p_{T} \leq 0.8 \mathrm{GeV} / \mathrm{c},|y|<0.5$ for different $\sqrt{s_{N N}}$ is shown in Fig. 21a.

The moments in the form $\kappa \sigma^{2}=\kappa_{4} / \kappa_{2}$ are shown from a previous STAR publication [84] in Fig. 21b while a plot, alleged to be of this same data, presented in the Lattice QCD theory publication that generated the press-release, is shown in Fig. 21. [85]; and a plot of the $\kappa \sigma^{2}$ from the data of Fig. 21k, combined with the results from Fig. 21b, is shown in Fig. 21d [83]. There are many interesting issues to be gleaned from Fig. 21 .

The data point at $20 \mathrm{GeV}$ in Fig. 21k is not the published one from (b), as stated in the caption [85], but the one from (d), which is different and with a much larger error. This, in

\footnotetext{
${ }^{3}$ Note that factorial Cumulants, also known as "Mueller moments" [82, have been used previously for multiplicity distributions.
} 
my opinion, makes the data point look better compared to the predicted discontinuous value of $\kappa \sigma^{2}=-1.5$ for the critical point at $20 \mathrm{GeV}$ (open crosses) in contrast to the predictions of 1.0 for both 62.4 and $200 \mathrm{GeV}$. The published measurements in (b) together with the new measurements in (d) are all consistent with $\kappa \sigma^{2}=1$; but clearly indicate the need for a better measurement at $\sqrt{s_{N N}}=20 \mathrm{GeV}$. Apart from these issues, the main problem of comparing Lattice QCD "data" to experimental measurements is that it is like comparing peaches to fish, since the prediction is the result of derivatives of the log of the calculated partition function of an idealized system, which may have little bearing on what is measured using finite sized nuclei in an experiment with severe kinematic cuts. Maybe this is too harsh a judgement; but since this is the first such comparison (hence the press release), perhaps the situation will improve in the future.

When I first saw the measured distributions in Fig. 217 in 2011, my immediate reaction was that STAR should fit them to Negative Binomial distributions (NBD) so that they would know all the Cumulants. However, I subsequently realized that my favorite 3 distributions for integer random variables, namely, Poisson, Binomial, and Negative Binomial, are all defined only for positive integers, while the number of net-protons on an event can be negative as well as positive, especially at higher c.m. energies. This is why somebody should work out the mathematical statistics of the net proton distribution as we did in PHENIX for the distribution of the difference in foreground (opposite charge) and background (like charge) di-lepton events when both are Poisson distributed [86]. Until then, it is instructive to compare the values of $\kappa \sigma^{2}$ in Fig. 21 to those from the well-known distributions: Poisson, $\kappa \sigma^{2}=1$; Binomial, $\kappa \sigma^{2}=1-6 p+6 p^{2}<1$; Negative Binomial, $\kappa \sigma^{2}=1+6 \mu / k+6 \mu^{2} / k^{2}>1$; Gaussian, all Cumulants $=0$ for $k>2$, so $\kappa \sigma^{2}=0$. The data favor Poisson (i.e. no correlation) everywhere, with some hint of Binomial. Nevertheless, if a future measurement would show a significant huge discontinuity of $\kappa \sigma^{2}$ similar to the theoretical prediction at $\sqrt{s_{N N}}=20 \mathrm{GeV}$, then even I would admit that such a discovery would deserve a press release, maybe more!

\subsection{The future measurement has appeared without a press release.}

In the intervening period since 2011, the STAR collaboration has improved the preliminary measurements to a publication [87] and has improved the analysis by comparing to both Poisson and Negative Binomial distributions which involved finding the formula for the Cumulants of the difference between two distributions [88. The first four Cumulants of the Poisson, Binomial and Negative Binomial distributions are given in Table 1. These three distributions fall into the class of "integer valued Lévy processes [89]" for which the Cumulants $\kappa_{j}$ for the difference between two such distributions, $P(m)=P^{+}(m)-P^{-}(m)$ with Cumulants $\kappa_{j}^{+}$and $\kappa_{j}^{-}$, respectively, are [88, 89]:

$$
\kappa_{j}=\kappa_{j}^{+}+(-1)^{j} \kappa_{j}^{-}
$$

Figure 22 [87] shows the STAR measurements of Cumulants of the net charge $\left(N^{+}-N^{-}\right)$ distributions from the "number of positive $\left(N^{+}\right)$and negative $\left(N^{-}\right)$charged particles within $|\eta|<$ 0.5 and $0.2<p_{T}<2.0 \mathrm{GeV} / \mathrm{c}$ on each event (after removing protons and antiprotons with $p_{T}<400$ $\mathrm{MeV} / \mathrm{c}$ ) [87]". The corresponding Poisson and NBD Cumulants were calculated from the measured mean, $\mu$, and variance, $\sigma^{2}$, of the $N^{+}$and $N^{-}$distributions, respectively, and then calculated using Eq. 5. In contrast to Fig. 21, no non-monotonic behavior with $\sqrt{s_{N N}}$ is observed (or claimed) and the measurements of $S \sigma$ and $\kappa \sigma^{2}$ are all above the Poisson baseline. The $S \sigma$ measurements clearly favor the NBD. The new results indicate that the search for a QCD critical point may not be as easy as originally assumed. 
Table 1: Cumulants for Poisson, Binomial and Negative Binomial Distributions

\begin{tabular}{llll}
\hline Cumulant & Poisson & Binomial & Negative Binomial \\
\hline$\kappa_{1}=\mu$ & $\mu$ & $n p$ & $\mu$ \\
$\kappa_{2}=\mu_{2}=\sigma^{2}$ & $\mu$ & $\mu(1-p)$ & $\mu(1+\mu / k)$ \\
$\kappa_{3}=\mu_{3}$ & $\mu$ & $\sigma^{2}(1-2 p)$ & $\sigma^{2}(1+2 \mu / k)$ \\
$\kappa_{4}=\mu_{4}-3 \kappa_{2}^{2}$ & $\mu$ & $\sigma^{2}\left(1-6 p+6 p^{2}\right)$ & $\sigma^{2}\left(1+6 \mu / k+6 \mu^{2} / k^{2}\right)$ \\
\hline$S \equiv \kappa_{3} / \sigma^{3}$ & $1 / \sqrt{\mu}$ & $(1-2 p) / \sigma$ & $(1+2 \mu / k) / \sigma$ \\
$\kappa \equiv \kappa_{4} / \kappa_{2}^{2}$ & $1 / \mu$ & $\left(1-6 p+6 p^{2}\right) / \sigma^{2}$ & $\left(1+6 \mu / k+6 \mu^{2} / k^{2}\right) / \sigma^{2}$ \\
$S \sigma$ & 1 & $(1-2 p)$ & $(1+2 \mu / k)$ \\
$\kappa \sigma^{2}$ & 1 & $\left(1-6 p+6 p^{2}\right)$ & $\left(1+6 \mu / k+6 \mu^{2} / k^{2}\right)$ \\
\hline
\end{tabular}

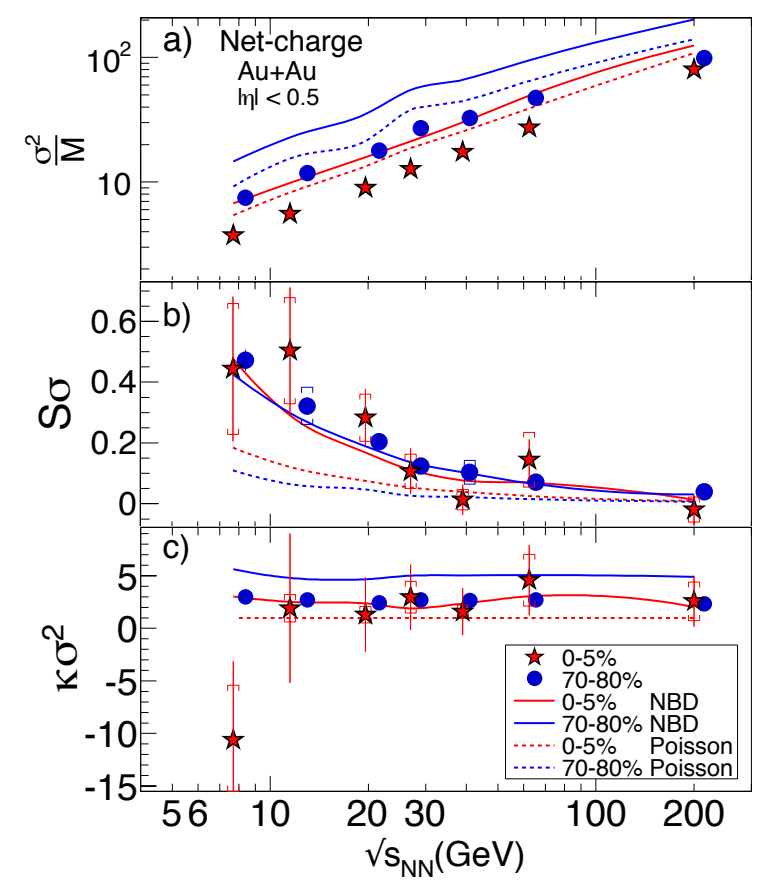

Figure 22: $\sqrt{s_{N N}}$ dependence of combinations of net-charge Cumulants in Au+Au from STAR [87, where in (a) $M$ is used to represent the mean, $\mu$.

\section{$7 \quad$ Hard Scattering as an in-situ probe}

One of the most important innovations at RHIC was the use of hard scattering as an in-situ probe of the medium produced in $\mathrm{A}+\mathrm{A}$ collisions by the effect of the medium on outgoing hard-scattered partons produced by the initial $\mathrm{A}+\mathrm{A}$ collision. This was observed primarily via inclusive single particle production at large transverse momentum $\left(p_{T}\right)$ or by two-particle correlations with a high $p_{T}$ trigger. The use of hard-scattering to probe the thermal or "soft" medium produced in RHI collisions was stimulated by pQCD studies [90] of the energy loss of partons produced by hard scattering, "with their color charge fully exposed", in traversing a medium "with a large density of similarly exposed color charges". The conclusion was that "Numerical estimates of the loss suggest that it may be significantly greater in hot matter than in cold. This makes the magnitude of the 
radiative energy loss a remarkable signal for QGP formation" [90]. In addition to being a probe of the QGP the fully exposed color charges allow the study of parton-scattering with $Q^{2} \ll 1-5$ $(\mathrm{GeV} / \mathrm{c})^{2}$ in the medium where new collective QCD effects may possibly be observed.

The hard-scattering takes place in the initial collision of the highly Lorentz contracted nuclei. The scattered partons which emerge near $90^{\circ}$ to the collision axis (the sweet-spot for such observations) pass through the medium formed and then fragment to jets of particles which are detected (Fig. 23). The most likely constituent reactions are: $g+g \rightarrow g+g, g+q \rightarrow g+q, q+q \rightarrow q+q$,
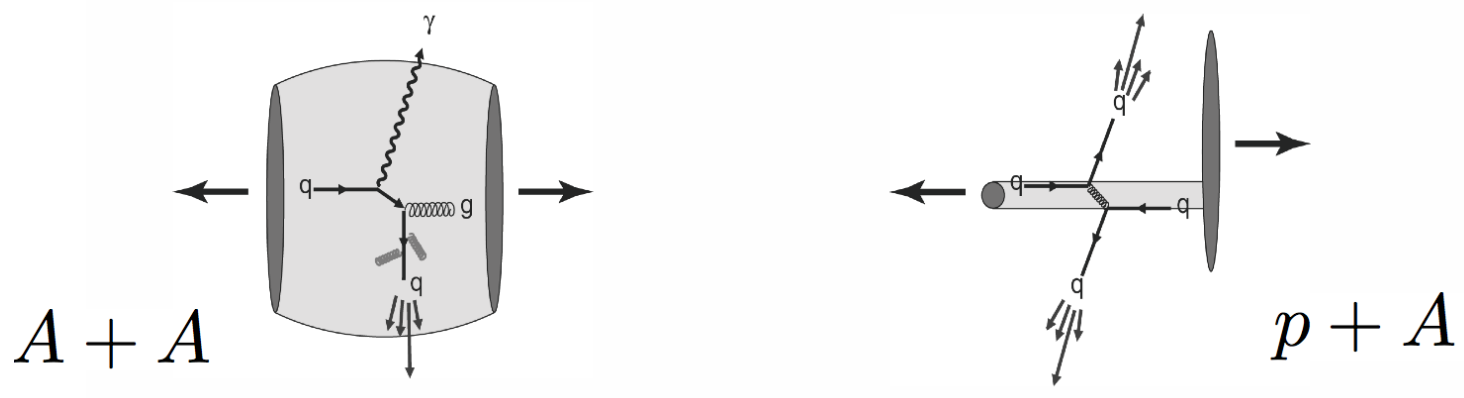

Figure 23: Schematic drawing of hard-scatterings in relativistic $\mathrm{A}+\mathrm{A}$ and $\mathrm{p}+\mathrm{A}$ collisions.

and $g+q \rightarrow \gamma+q$ (shown). This last reaction [91], called direct- $\gamma$ production or the inverse QCD Compton effect, is the most beautiful because the $\gamma$-ray participates directly in the hard scattering, then emerges from the medium without interacting and can be measured with high precision. No fragmentation is involved and, in fact, these direct- $\gamma$ are distinguished from e.g. $\gamma$ rays from fragmenting partons because they are isolated, with no accompanying fragments. Triggering on a direct- $\gamma$ of a given $\hat{p}_{T_{t}}$ provides a 'beam' of partons with exactly opposite (thus well-known) initial $\hat{p}_{T}=-\hat{p}_{T_{t}}$, so that the effect of the medium can be determined by measuring, for instance, the ratio of the transverse momentum $\hat{p}_{T_{a}}$ of the jet from the away-parton to that of the direct- $\gamma$ trigger, denoted $\hat{x}_{h}=\hat{p}_{T_{a}} / \hat{p}_{T_{t}}$, or equivalently, the fractional jet imbalance, $1-\hat{x}_{h}$, as used by CMS at LHC [16].

Since hard-scattering at high $p_{T}>2 \mathrm{GeV} / \mathrm{c}$ is point-like, with distance scale $1 / p_{T}<0.1 \mathrm{fm}$, the cross section in $\mathrm{p}+\mathrm{A}(\mathrm{A}+\mathrm{A})$ collisions, compared to $\mathrm{p}-\mathrm{p}$, should be larger by the relative number of possible point-like encounters, a factor of $A\left(A^{2}\right)$ for $\mathrm{p}+\mathrm{A}(\mathrm{A}+\mathrm{A})$ minimum bias collisions. When the impact parameter or centrality of the collision is defined, the proportionality factor becomes $\left\langle T_{A A}\right\rangle$, the average overlap integral of the nuclear thickness functions. Measurements in $\mathrm{p}+\mathrm{A}$ (or $\mathrm{d}+\mathrm{A}$ ) collisions, where no (or negligible) medium is produced, allow correction for any modification of the nuclear structure function from an incoherent superposition of proton and neutron structure functions.

\subsection{Jet quenching - suppression of high $p_{T}$ particles}

The discovery, at RHIC [92, that $\pi^{0}$ 's produced at large transverse momenta are suppressed in central $\mathrm{Au}+\mathrm{Au}$ collisions by a factor of $\sim 5$ compared to point-like scaling from $\mathrm{p}$-p collisions is arguably the major discovery in Relativistic Heavy Ion Physics. For $\pi^{0}$ (Fig. 24a) [93] the hard-scattering in p-p collisions is indicated by the power law behavior $p_{T}^{-n}$ for the invariant cross section, $E d^{3} \sigma / d p^{3}$, with $n=8.1 \pm 0.05$ for $p_{T} \geq 3 \mathrm{GeV} / \mathrm{c}$. The $\mathrm{Au}+\mathrm{Au}$ data at a given $p_{T}$ can be characterized either as shifted lower in $p_{T}$ by $\delta p_{T}$ from the point-like scaled p-p data at $p_{T}^{\prime}=p_{T}+\delta p_{T}$, or shifted down in magnitude, i.e. suppressed. In Fig. $24 \mathrm{~b}$, the suppression of the 

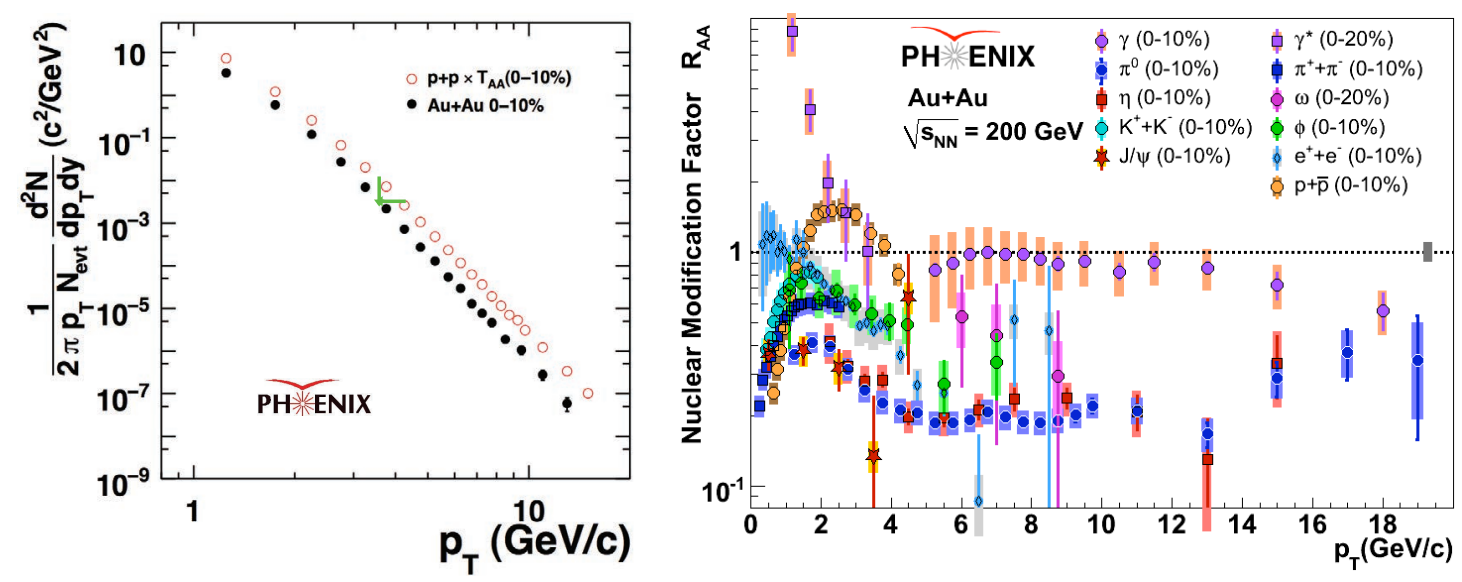

Figure 24: a) (left) Log-log plot of invariant yield of $\pi^{0}$ at $\sqrt{s_{N N}}=200 \mathrm{GeV}$ as a function of transverse momentum $p_{T}$ in p-p collisions multiplied by $\left\langle T_{A A}\right\rangle$ for Au+Au central $(0-10 \%)$ collisions compared to the $\mathrm{Au}+\mathrm{Au}$ measurement $\left[93\right.$. b) (right) $R_{A A}\left(p_{T}\right)$ for all identified particles so far measured by PHENIX in $\mathrm{Au}+\mathrm{Au}$ central collisions at $\sqrt{s_{N N}}=200 \mathrm{GeV}$.

many identified particles measured by PHENIX at RHIC is presented as the Nuclear Modification Factor, $R_{A A}\left(p_{T}\right)$, the ratio of the yield of e.g. $\pi$ per central $\mathrm{Au}+\mathrm{Au}$ collision (upper 10\%-ile of observed multiplicity) to the point-like-scaled p-p cross section, where $\left\langle T_{A A}\right\rangle$ is the average overlap integral of the nuclear thickness functions:

$$
R_{A A}\left(p_{T}\right)=\frac{d^{2} N_{A A}^{\pi} / d p_{T} d y N_{A A}}{\left\langle T_{A A}\right\rangle d^{2} \sigma_{p p}^{\pi} / d p_{T} d y} .
$$

The striking differences of $R_{A A}\left(p_{T}\right)$ in central $\mathrm{Au}+\mathrm{Au}$ collisions for the many particles measured by PHENIX (Fig. 24b) illustrates the importance of particle identification for understanding the physics of the medium produced at RHIC. Most notable are: the equal suppression of $\pi^{0}$ and $\eta$ mesons by a constant factor of $5\left(R_{A A}=0.2\right)$ for $4 \leq p_{T} \leq 15 \mathrm{GeV} / \mathrm{c}$, with suggestion of an increase in $R_{A A}$ for $p_{T}>15 \mathrm{GeV} / \mathrm{c}$; the equality of suppression of direct-single $e^{ \pm}$(from heavy quark $(c, b$ ) decay) and $\pi^{0}$ at $p_{T} \gtrsim 5 \mathrm{GeV} / \mathrm{c}$; the non-suppression of direct- $\gamma$ for $p_{T} \geq 4 \mathrm{GeV} / \mathrm{c}$; the exponential rise of $R_{A A}$ of direct- $\gamma$ for $p_{T}<2 \mathrm{GeV} / \mathrm{c}$ [94, which is totally and dramatically different from all other particles and attributed to thermal photon production by many authors (e.g. see citations in Ref. [94]). For $p_{T} \gtrsim 4 \mathrm{GeV} / \mathrm{c}$, the hard-scattering region, the fact that all hadrons are suppressed, but direct- $\gamma$ are not suppressed, indicates that suppression is a medium effect on outgoing colorcharged partons likely due to energy loss by coherent Landau-Pomeranchuk-Migdal radiation of gluons, predicted in pQCD [90], which is sensitive to properties of the medium. Measurements of two-particle correlations [12 confirm the loss of energy of the away-jet relative to the trigger jet in $\mathrm{Au}+\mathrm{Au}$ central collisions compared to p-p collisions. However, there are still many details which remain to be understood, such as the apparent suppression of direct- $\gamma$ for $p_{T} \gtrsim 18 \mathrm{GeV} / \mathrm{c}$, approaching that of the $\pi^{0}$. Interesting new results have extended and clarified these observations.

An improved measurement of $\pi^{0}$ production in $\mathrm{Au}+\mathrm{Au}$ and p-p collisions by PHENIX [95] now clearly shows a significant increase of $R_{A A}$ (decrease in suppression) with increasing $p_{T}$ over the range $7<p_{T}<20 \mathrm{GeV} / \mathrm{c}$ for $0-5 \%$ central $\mathrm{Au}+\mathrm{Au}$ collisions at $\sqrt{s_{N N}}=200 \mathrm{GeV}$ (Fig. $25 \mathrm{a}$ ). Comparisons of the suppression of non-identified hadrons in $\sqrt{s_{N N}}=2.76 \mathrm{TeV} \mathrm{Pb}+\mathrm{Pb}$ collisions at $\mathrm{LHC}$ to the RHIC $\mathrm{Au}+\mathrm{Au} \pi^{0}$ data are also very interesting. This is shown both in terms of the suppression, $R_{A A}\left(p_{T}\right)$ (Fig. 25a), and the fractional shift in the $p_{T}$ spectrum $\delta p_{T} / p_{T}^{\prime}$ (Fig. 25b). Interestingly, despite more than a factor of 20 higher c.m. energy, the ALICE $R_{A A}$ data from 

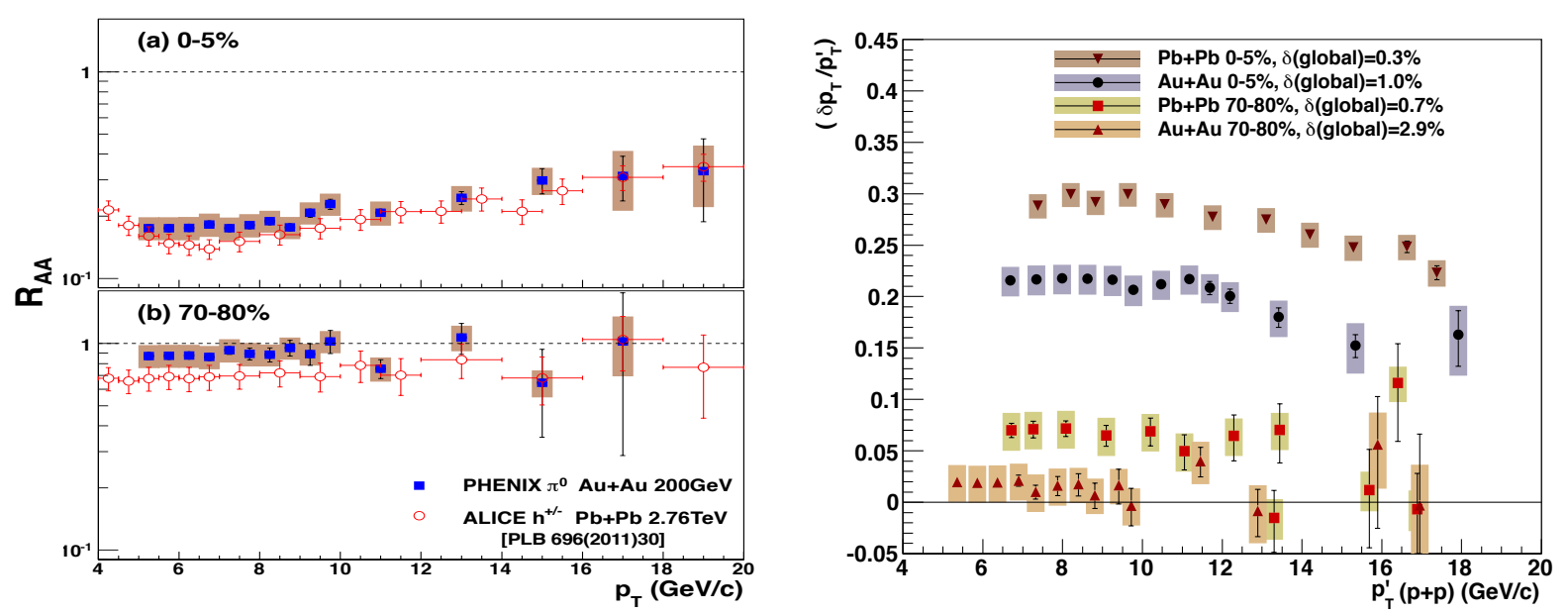

Figure 25: a) (left) $R_{A A}$ of $\pi^{0}$ in $\sqrt{s_{N N}}=200 \mathrm{GeV}$ central (0-5\%) and peripheral (70-80\%) Au+Au collisions [95] at RHIC compared to non-identified charged hadron $\left(\mathrm{h}^{ \pm}\right) R_{A A}$ in $\sqrt{s_{N N}}=2.76 \mathrm{TeV}$ $\mathrm{Pb}+\mathrm{Pb}$ collisions at LHC. [96] b) (right) Fractional shift of $p_{T}$ spectrum $\delta p_{T} / p_{T}^{\prime}$ vs. $p_{T}^{\prime}$ (p-p) calculated by PHENIX [95] for RHIC and LHC.

LHC [96] are nearly identical to the RHIC measurement [95] for $5<p_{T}<20 \mathrm{GeV} / \mathrm{c}$. Since the exponent of the power-law at LHC $(n \approx 6)$ is flatter than at RHIC $(n \approx 8)$, a $\sim 40 \%$ larger shift $\delta p_{T} / p_{T}^{\prime}$ in the spectrum from p-p to $\mathrm{A}+\mathrm{A}$ is required at LHC (Fig. $25 \mathrm{~b}$ ) to get the same $R_{A A}$, which likely indicates $\sim 40 \%$ larger fractional energy loss at LHC in this $p_{T}$ range due to the probably hotter and denser medium.

In Fig. 26, these measurements are combined with the previous measurements at RHIC for $\sqrt{s_{N N}}=39$ and $62.4 \mathrm{GeV}$ [97] to reveal a systematic increase of $\delta p_{T} / p_{T}^{\prime}$ in central A+A collisions

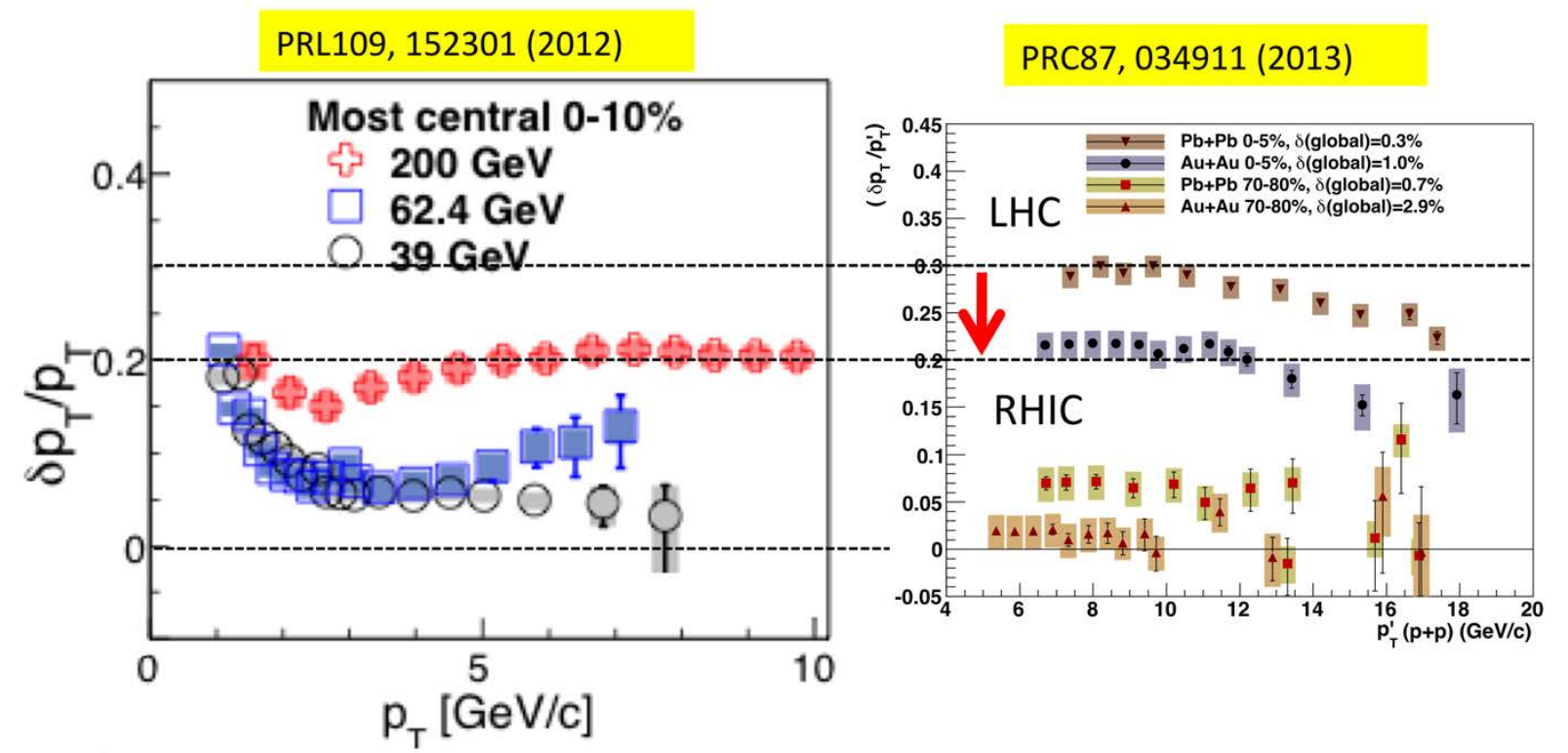

Figure 26: Fractional shift of $p_{T}$ spectrum $\delta p_{T} / p_{T}^{\prime}$ in central A+A collisions from $\sqrt{s_{N N}}=39 \mathrm{GeV}$ to $2.76 \mathrm{TeV}$ 

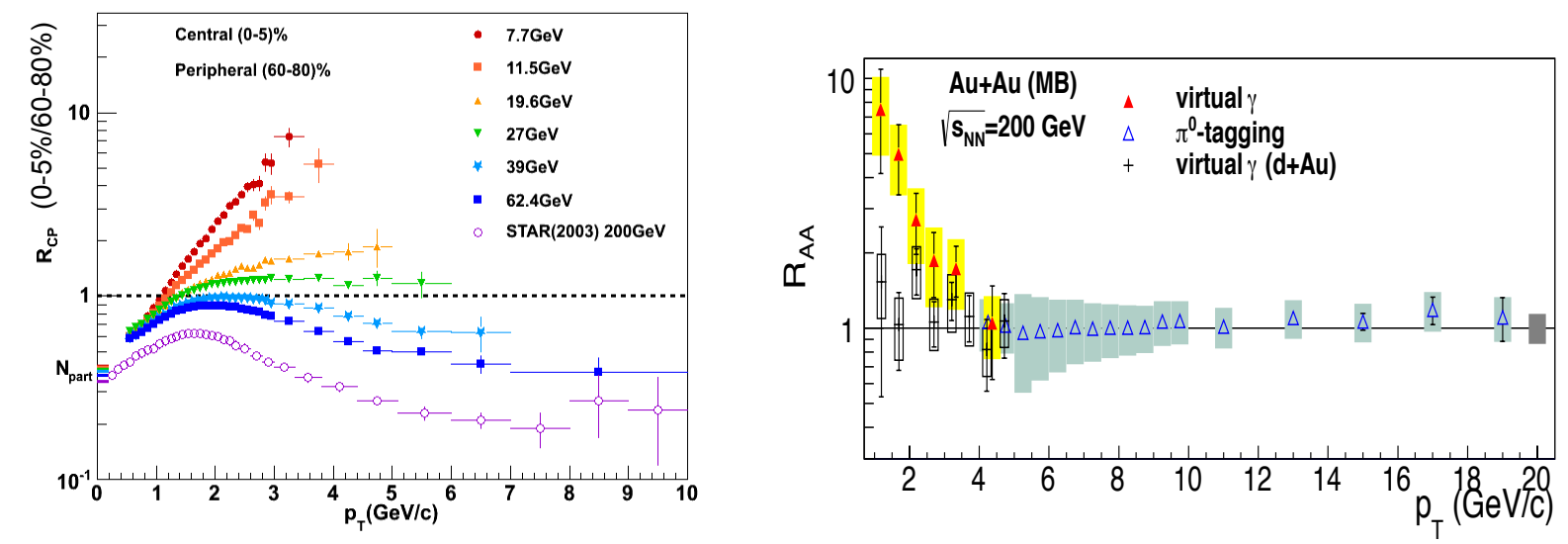

Figure 27: a) (left) STAR $R_{\mathrm{CP}}\left(p_{T}\right)$ for $h^{ \pm}$as a function of $\sqrt{s_{N N}}$ in $\mathrm{Au}+\mathrm{Au}$ collisions [98] . b)(right) PHENIX $R_{A A}\left(p_{T}\right)$ of direct- $\gamma$ in $\mathrm{d}+\mathrm{Au}$ and $\mathrm{Au}+\mathrm{Au}$ minimum bias collisions at $\sqrt{s_{N N}}=200$ $\mathrm{GeV}$ [99].

at $p_{T}^{\prime}=7 \mathrm{GeV} / \mathrm{c}$, going from $5 \%$ to $30 \%$ over the c.m. energy range $\sqrt{s_{N N}}=39 \mathrm{GeV}$ to $2.76 \mathrm{TeV}$. Measurements by STAR (Fig. 27a) [98] of the evolution of charged hadron suppression with $\sqrt{s_{N N}}$ in $\mathrm{Au}+\mathrm{Au}$ collisions, using the variable $R_{C P}=\left(R_{A A}^{0-5 \%} / R_{A A}^{60-80 \%}\right)$, which does not require the p-p cross section (see Eq. 6) but is usually smaller than $R_{A A}$ [5], show the transition from suppression $\left(R_{\mathrm{CP}}<1\right)$ to enhancement $\left(R_{\mathrm{CP}}>1\right)$ for $\sqrt{s_{N N}} \lesssim 27 \mathrm{GeV}$.

Improved measurements of direct- $\gamma$ production in $\mathrm{p}-\mathrm{p}, \mathrm{dAu}$ and $\mathrm{Au}+\mathrm{Au}$ collisions by PHENIX 99 ] show several interesting results. In Fig. $27 \mathrm{~b}$, new measurements of $R_{A A} \approx 1$ for $\mathrm{d}+\mathrm{Au}$ using internal conversions in the thermal region, $p_{T}<4 \mathrm{GeV}$, reinforce the uniqueness of the exponential rise of the $\mathrm{Au}+\mathrm{Au}$ minimum bias photon spectrum, thus confirming that the exponential for $p_{T}<4$ $\mathrm{GeV} / \mathrm{c}$ in $\mathrm{Au}+\mathrm{Au}$ is a hot matter effect, i.e. thermal photon production. Also in Fig. 27p, improved measurements of real direct- $\gamma$ in $\mathrm{Au}+\mathrm{Au}$ collisions, by eliminating background from $\gamma$-rays associated with a second $\gamma$ in the $\pi^{0}$ mass range $\left(\pi^{0}\right.$ tagging), no longer show an "apparent suppression" but are consistent with $R_{A A}=1$ out to $20 \mathrm{GeV} / \mathrm{c}$.

My favorite direct- $\gamma$ result in 2012 was the improved PHENIX measurement in p-p collisions at $\sqrt{s}=200 \mathrm{GeV}$ out to $p_{T}=25 \mathrm{GeV} / \mathrm{c}$ [100], in excellent agreement with pQCD. A more direct way to show this without a detailed theory calculation is to use $x_{T}$ scaling. Figure 28 a shows $x_{T}$ scaling for all presently existing direct- $\gamma$ data 4 , with $n_{\text {eff }}=4.5$, very close to the pure-scaling parton-parton Rutherford scattering result of $n_{\text {eff }}=4.0$ (Fig. 28b). The deviation of the data points in Fig. 28b from the universal curve for $\sqrt{s}>38.7 \mathrm{GeV}$ is an illustration of the non-scaling of the coupling constant, structure and fragmentation functions in QCD - what I like to call "QCD in action". For $\sqrt{s} \leq 38.7 \mathrm{GeV}$, the deviation of the data from the universal curve in Fig. 28 a (and from pQCD) is claimed to be due to the $k_{T}$ effect (transverse momentum of the quarks in a nucleon).

\subsection{Two-Particle Correlations and Fragmentation Functions}

The key to measuring the fragmentation function of the jet of particles from a hard-scattered parton is to know the energy of the original parton which fragments, as pioneered at LEP [101]. Since the $p_{T}^{\gamma}$ of a direct- $\gamma$ can be measured very precisely, the fragmentation function of the jet from the

\footnotetext{
${ }^{4}$ This includes the PHENIX p-p direct-virtual- $\gamma$ measurement down to $p_{T} \approx 1 \mathrm{GeV} / \mathrm{c}$, further confirming the absence of a soft production mechanism for direct- $\gamma$ in p-p collisions [12].
} 

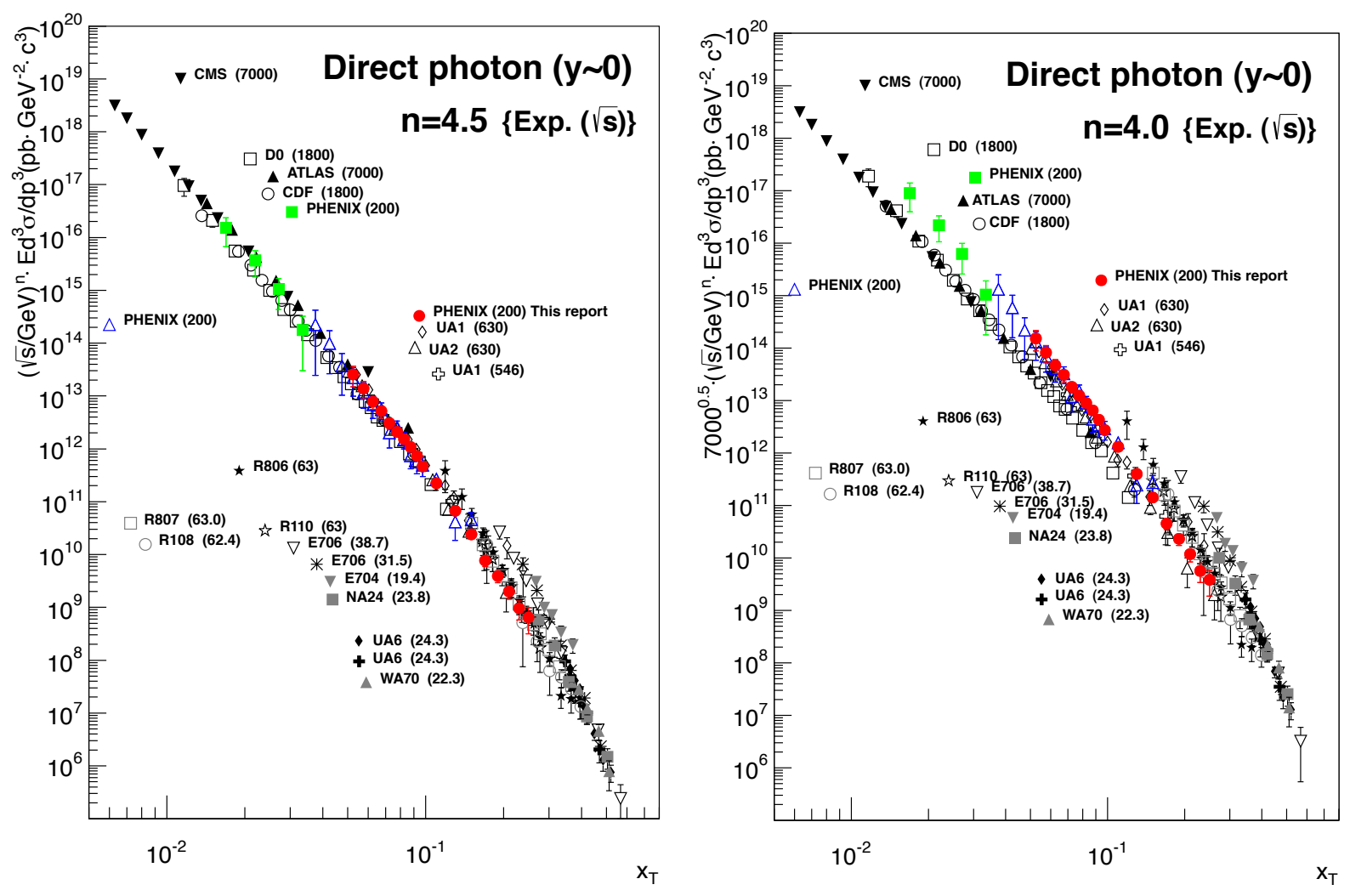

Figure 28: a)(left) $\sqrt{s}^{n_{\text {eff }}} \times E d^{3} \sigma / d p^{3}$, as a function of $x_{T}=2 p_{T} / \sqrt{s}$, with $n_{\text {eff }}=4.5$, for direct- $\gamma$ measurements in $\mathrm{p}-\mathrm{p}$ and $\overline{\mathrm{p}}$-p experiments at the $(\sqrt{s} \mathrm{GeV})$ indicated. [100]. b)(right) same as (a) with $n_{\text {eff }}=4.0$

away quark in the reaction $g+q \rightarrow \gamma+q$ can be measured by the direct- $\gamma-h$ correlations (where $h$ represents charged hadrons opposite in azimuth to the direct- $\gamma$ ) because the $p_{T}$ of the away-quark at production is equal and opposite to $p_{T}^{\gamma}$, thus known to high precision (modulo a small $k_{T}$-smearing effect). A further advantage is that the identity of the away-quark fragmenting to the jet is also known to reasonable precision: 8/1 u-quark, in $\mathrm{p}+\mathrm{p}$ collisions (maybe $8 / 2$ if the $\bar{q}+q \rightarrow \gamma+g$ channel is included) and $\sim 3 / 1$ in $\mathrm{A}+\mathrm{A}$ collisions (not counting the $\bar{q}+q \rightarrow \gamma+g$ channel). The main disadvantage is the low rate since the $\gamma-q$ vertex is electromagnetic. Two-particle correlations are analyzed in terms of the two variables [102]: $p_{\text {out }}=p_{T} \sin (\Delta \phi)$, the out-of-plane transverse momentum of a track; and $x_{E}$, where:

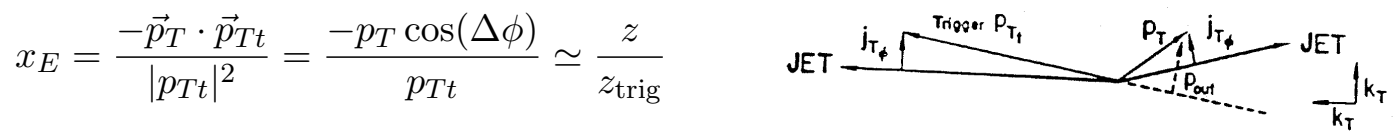

$z_{\text {trig }} \simeq p_{T t} / p_{T \text { jet }}$ is the fragmentation variable of the trigger jet, and $z$ is the fragmentation variable of the away jet. Note that $x_{E}$ would equal the fragmenation fraction $z$ of the away jet, for $z_{\operatorname{trig}} \rightarrow 1$, if the trigger and away jets balanced transverse momentum. The beauty of direct- $\gamma$ for this purpose is that $z_{\text {trig }} \equiv 1$.

Following the approach of Borghini and Wiedemann 103 who predicted the medium modification of fragmentation functions in the hump-backed or $\xi=\ln (1 / z)$ representation, PHENIX measured $x_{E}$ distributions in p-p collisions [104] and converted them to the $\xi=-\ln x_{E}$ representation (Fig. 29 a) which are in quite excellent agreement with the dominant $u$-quark fragmentation func- 


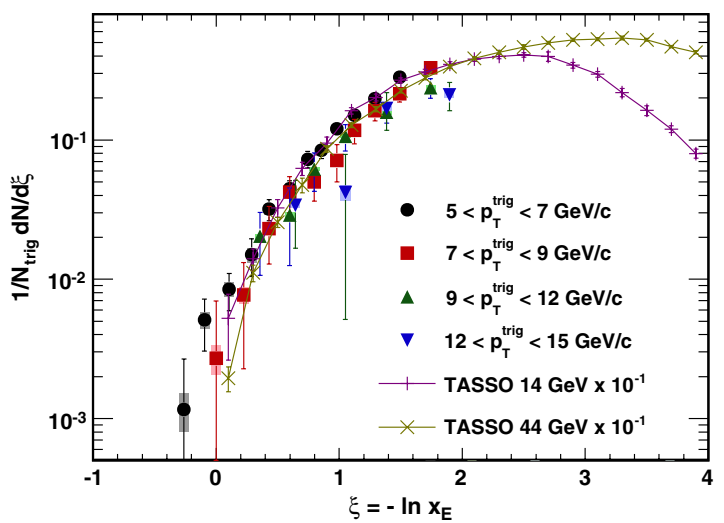

Figure 29: a) (left) PHENIX measurement [104] of direct- $\gamma$-h correlations in p$\mathrm{p}$ collisions at $\sqrt{s}=200 \mathrm{GeV}$ in the variable $\xi=-\ln x_{E} \approx-\ln \left(p_{T}^{h} / p_{T}^{\gamma}\right)$ compared to fragmentation functions measured in $e^{+} e^{-}$ collisions at $\sqrt{s}=14$ and $44 \mathrm{GeV}$ by TASSO [105]. b)(right)-(top) $\xi$ distributions of direct- $\gamma$-h correlations in $\mathrm{Au}+\mathrm{Au}$ and $\mathrm{p}-$ $\mathrm{p}$ collisions at $\sqrt{s}=200 \mathrm{GeV}$. c) (right)(bottom) Ratio of the $\mathrm{Au}+\mathrm{Au} / \mathrm{p}-\mathrm{p}$ distributions, $I_{A A}(\xi)$ when the away side azimuthal range is restricted as indicated [106]
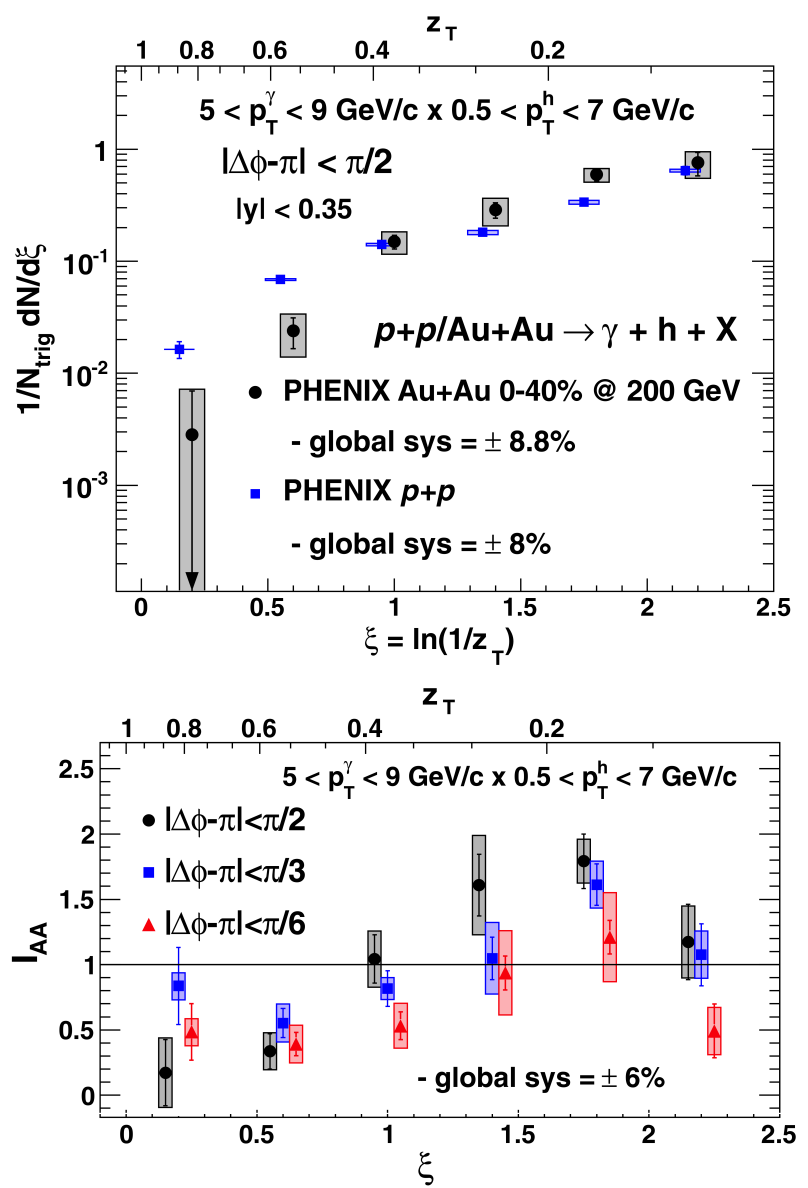

tions measured in $e^{+} e^{-}$collisions at $\sqrt{s} / 2=7$ and $22 \mathrm{GeV}$ [105], which cover a comparable range in jet energy. In 2013, improved measurements by PHENIX [106] in both p-p and Au+Au collisions (Fig. 29b) now indicate a significant modification of the fragmentation function in $\mathrm{Au}+\mathrm{Au}(0-40 \%)$ central collisions compared to p-p, with an enhancement at low $z_{T}=p_{T}^{h} / p_{T}^{\gamma}$ (large $\xi=-\ln z_{T}$ ) and a suppression at large $z_{T}$ (small $\xi$ ) which is more clearly seen as $I_{A A}(\xi)$, the ratio of the fragmentation functions in $\mathrm{Au}+\mathrm{Au} / \mathrm{pp}$ (Fig. 29k). As shown in Fig. 29k, restricting the away-side azimuthal range reduces the large $\xi>0.9\left(p_{T}^{h} \lesssim 3 \mathrm{GeV} / \mathrm{c}\right)$ enhancement but leaves the suppression at small $\xi<0.9$ relatively unchanged, which shows that the large $\xi$ enhancement is predominantly at large angles, similar to the effect observed by CMS with actual jets. 107. Fragmentation functions from full jet reconstruction in $\mathrm{A}+\mathrm{A}$ collisions are not yet available at RHIC.

\subsection{Two-Particle Correlations and Jet Imbalance}

One of the important lessons learned at RHIC [102] about fragmentation functions is that the away-side $x_{E}$ distribution of particles opposite to a trigger particle (e.g. a $\pi^{0}$ ), which is itself the fragment of a jet, does not measure the fragmentation function, but, instead, measures the ratio of $\hat{p}_{T_{a}}$ of the away-parton to $\hat{p}_{T_{t}}$ of the trigger-parton and depends only on the same power $n$ as the invariant single particle spectrum:

$$
\left.\frac{d P}{d x_{E}}\right|_{p_{T_{t}}} \approx N(n-1) \frac{1}{\hat{x}_{h}} \frac{1}{\left(1+\frac{x_{E}}{\hat{x}_{h}}\right)^{n}} .
$$



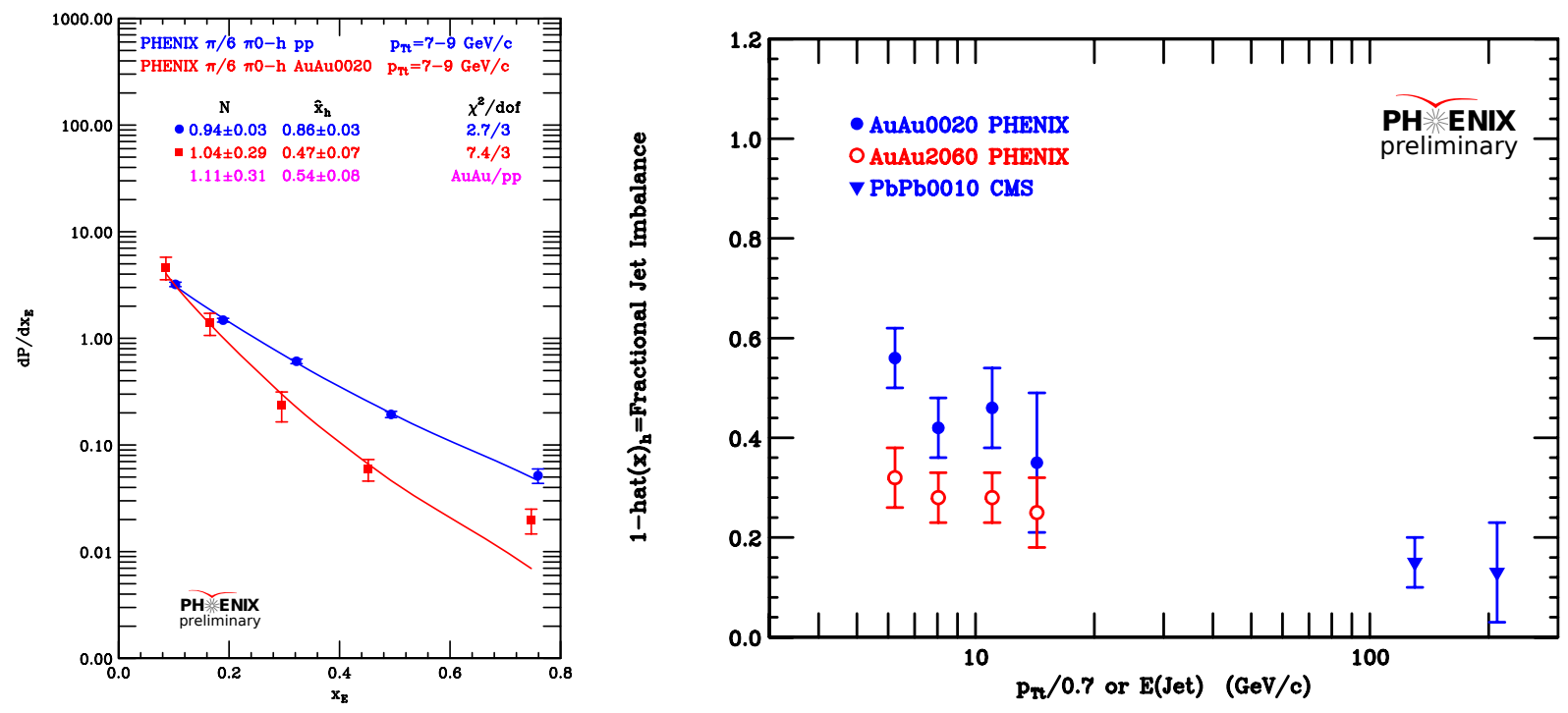

Figure 30: (left) a) $x_{E}$ distributions [109] from p-p (circles) and AuAu 0-20\% centrality (squares) for $p_{T_{t}}=7-9 \mathrm{GeV} / \mathrm{c}$, together with fits to Eq. 7 (solid lines) with parameters indicated. The ratios of the fitted parameters for $\mathrm{AuAu} / \mathrm{pp}$ are also given. b) (right) Fractional jet imbalance [109], $1-\hat{x}_{h}^{A A} / \hat{x}_{h}^{p p}$, for RHIC and CMS data.

This equation gives a simple relationship between the ratio, $x_{E} \approx p_{T_{a}} / p_{T_{t}} \equiv z_{T}$, of the transverse momenta of the away-side particle to the trigger particle, and the ratio of the transverse momenta of the away-jet to the trigger-jet, $\hat{x}_{h}=\hat{p}_{T_{a}} / \hat{p}_{T_{t}}$. PHENIX measurements [108] of the $x_{E}$ distributions of $\pi^{0}$-h correlations in p-p and $\mathrm{Au}+\mathrm{Au}$ collisions at $\sqrt{s_{N N}}=200 \mathrm{GeV}$ were fit to Eq. 7] (Fig. 30a) [109]. The steeper distribution in $\mathrm{Au}+\mathrm{Au}$ shows that the away parton has lost energy. The results for the fitted parameters are shown on the figure.

In general the values of $\hat{x}_{h}^{p p}$ do not equal 1 but range between $0.8<\hat{x}_{h}^{p p}<1.0$ due to $k_{T}$ smearing and the range of $x_{E}$ covered. In order to take account of the imbalance $\left(\hat{x}_{h}^{p p}<1\right)$ observed in the p-p data, the ratio $\hat{x}_{h}^{A A} / \hat{x}_{h}^{p p}$ is taken as the measure of the energy of the away jet relative to the trigger jet in $\mathrm{A}+\mathrm{A}$ compared to $\mathrm{p}-\mathrm{p}$ collisions. The fractional jet imbalance was also measured directly with reconstructed di-jets by the CMS collaboration at the $\mathrm{LHC}$ in $\mathrm{Pb}+\mathrm{Pb}$ central collisions at $\sqrt{s_{\mathrm{NN}}}=2.76 \mathrm{TeV}[16]$. There was also a large imbalance in p-p collisions due to the cuts on jet $\hat{p}_{T}$ that were used. I calculated $\hat{x}_{h}$ from their $\mathrm{p}-\mathrm{p}$ and $\mathrm{Pb}+\mathrm{Pb}$ results and compared these LHC values of $1-\hat{x}_{h}^{A A} / \hat{x}_{h}^{p p}$ to those from PHENIX (Fig. 30 b). Newer results in 2012 by CMS (Fig. 31] 110] significantly extended and improved their previous measurement and confirmed my correction [109].

In Figs. 30b and 31b, there is a large difference in fractional jet imbalance in the different $\hat{p}_{T}$ ranges covered by the RHIC and LHC measurements. This could be due to the difference in jet $\hat{p}_{T_{t}}$ between RHIC $(\sim 20 \mathrm{GeV} / \mathrm{c})$ and LHC $(\sim 200 \mathrm{GeV} / \mathrm{c})$, a difference in the properties of the medium, the difference in $n$ for the different $\sqrt{s_{N N}}$, or a problem with Eq. 7 which has not been verified by direct comparison to di-jets. In any case the strong $\hat{p}_{T}$ dependence of the fractional jet imbalance (apparent energy loss of a parton) also seems to disfavor purely radiative energy-loss in the QGP [90] and indicates that the details of energy loss in a QGP remain to be understood. Future measurements at both RHIC and LHC will need to sort out these issues by extending di-jet and two-particle correlation measurements to overlapping regions of $\hat{p}_{T}$. 

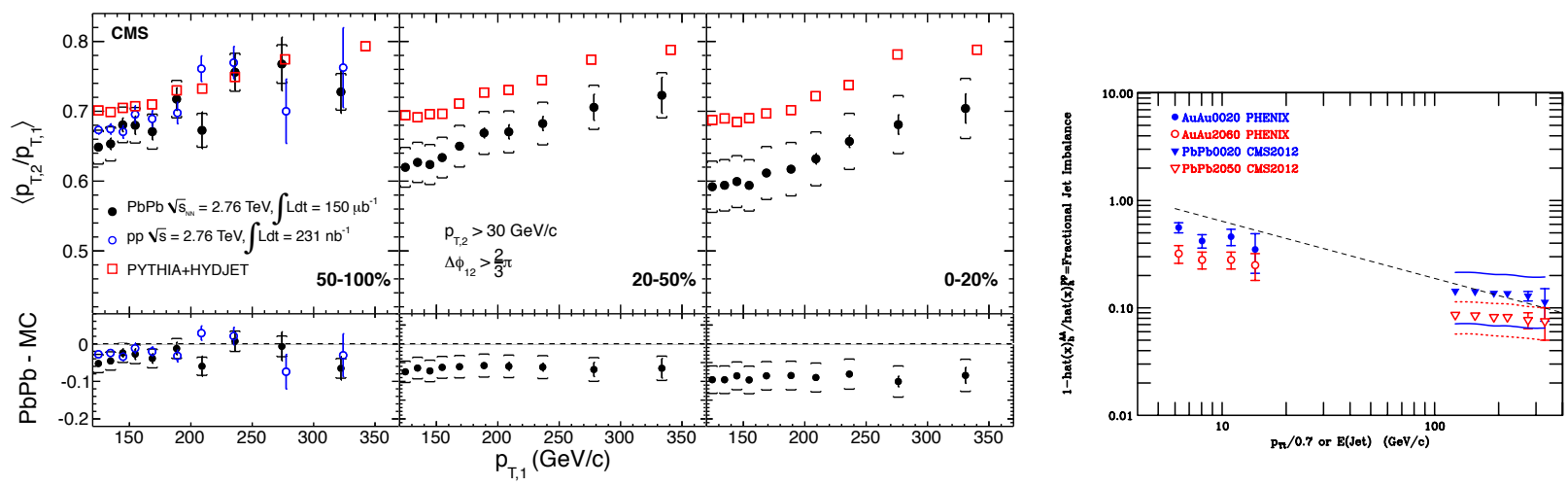

Figure 31: a) (left 3 panels) CMS [110] measurements of average di-jet transverse momentum ratio, $\hat{x}_{h}=\hat{p}_{T, 2} / \hat{p}_{T, 1}$, as a function of leading jet $\hat{p}_{T, 1}$ at $\sqrt{s_{N N}}=2.76 \mathrm{TeV}$ in p-p collisions and for 3 centralities in $\mathrm{Pb}+\mathrm{Pb}$ collisions, as well as simulated $\mathrm{p}$-p di-jets embedded in heavy ion events. b) (right) Fractional jet imbalance [109], $1-\hat{x}_{h}^{A A} / \hat{x}_{h}^{p p}$, for the RHIC data from Fig. 30 with CMS measurement from (a). The solid (dotted) lines represent the systematic uncertainty of the CMS 0-20\% (20-50\%) results. The dashed line is my estimate of the Fractional Jet Imbalance vs. E(Jet) at $\sqrt{s_{N N}}=2.76 \mathrm{TeV}$ made the previous year from Fig. $30 \mathrm{p}$.

\section{Anisotropic flow $\left(v_{2}\right)$ of direct- $\gamma$}

Although direct- $\gamma$ production [91] is the most beautiful QCD subprocess, it has a very serious problem: an overwhelming background of photons from high $p_{T} \pi^{0} \rightarrow \gamma+\gamma$ and $\eta \rightarrow \gamma+\gamma$ decays makes it a very difficult experiment. One must measure all the background sources: $\pi^{0}, \eta, \ldots$, and calculate their contributions to the inclusive $\gamma$-ray spectrum. In principle, the background can be calculated whatever the $p_{T}$ distribution of the $\pi^{0}$ and $\eta$. However nature has been kind in that the invariant cross section for hard-scattering is a power law, $d \sigma / p_{T} d p_{T} \propto 1 / p_{T}^{n}$, with $n=8.1 \pm 0.05$ at $\sqrt{s_{N N}}=200 \mathrm{GeV}$, for $\pi^{0}$ with $p_{T} \geq 3 \mathrm{GeV} / \mathrm{c}$ (Fig. 24a) [93]; also for $p_{T} \geq 3$ $\mathrm{GeV} / \mathrm{c}, \eta / \pi^{0}=0.48 \pm 0.03$ is a constant. This implies that for $\pi^{0} \rightarrow \gamma+\gamma$, the spectrum of decay photons has the same power as the parent $\pi^{0}$ so that the ratio at any $p_{T}$ is a constant:

$$
\left.\frac{\gamma}{\pi^{0}}\right|_{\pi^{0}}=\frac{2}{n-1}
$$

The resulting background inclusive $\gamma$ spectrum from $\pi^{0}$ and $\eta$ decays at $\sqrt{s_{N N}}=200 \mathrm{GeV}$ is:

$$
\gamma_{\text {background }} / \pi^{0} \approx(1+0.48 \times 0.39) \times 2 / 7.1=1.19 \times 2 / 7.1=0.334
$$

where 0.39 is the branching ratio for $\eta \rightarrow \gamma+\gamma$. In PHENIX we plot what we call the double ratio:

$$
R_{\gamma}=\left(\gamma_{\text {inclusive }} / \pi^{0}\right) /\left(\gamma_{\text {background }} / \pi^{0}\right)=\gamma_{\text {inclusive }} / \gamma_{\text {background }}
$$

where it is important to see the calculated $\gamma_{\text {background }} / \pi^{0}$ ratio, which usually comes from some opaque Monte Carlo program, to understand whether it makes sense according to Eqs. 8 and 9 .

Fig. 32 [111] shows $R_{\gamma}$ for real photons, measured in an EM calorimeter, and virtual photons, which are $e^{+} e^{-}$pairs from internal conversion of the direct- $\gamma$, with $0.12<m_{e e}<0.30 \mathrm{GeV} / \mathrm{c}^{2}$ where there is no background from $\pi^{0}$ Dalitz decay. This reduces the background by a factor of 

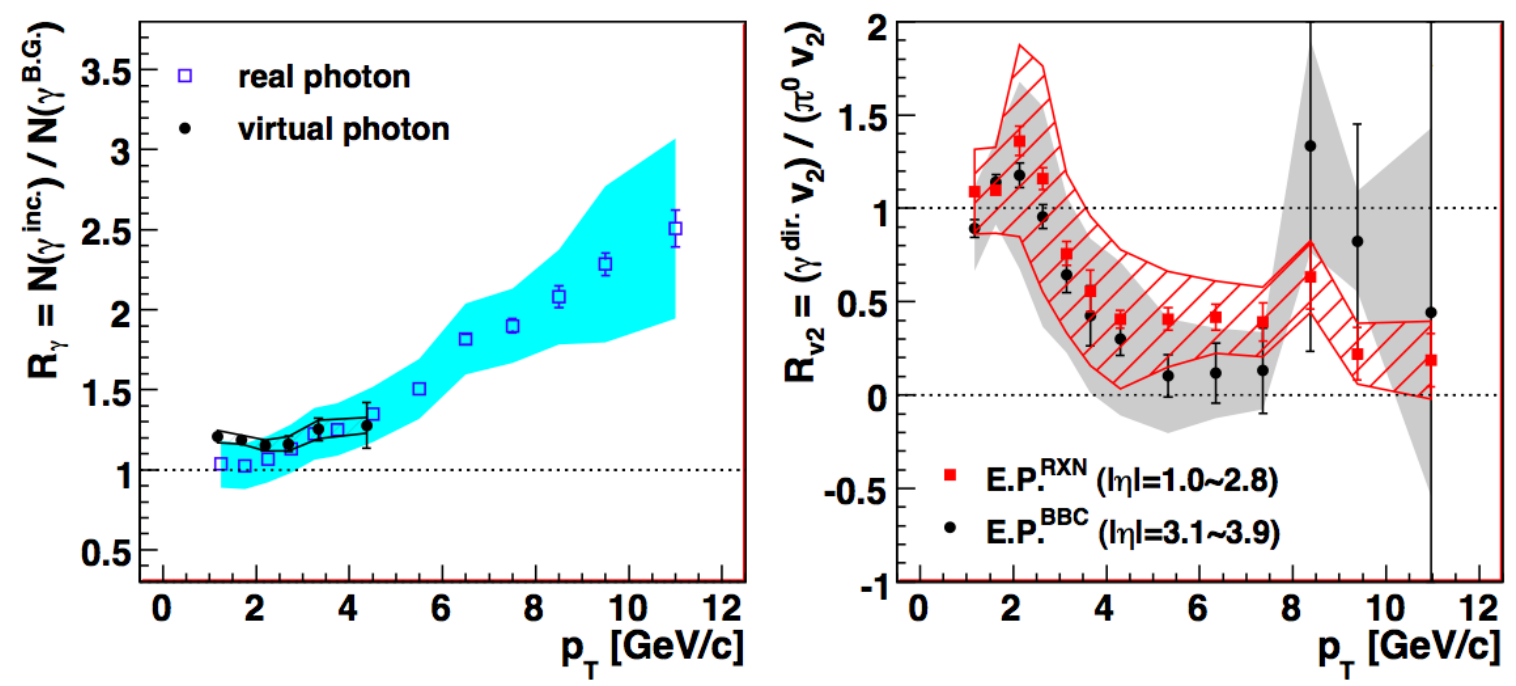

Figure 32: a) (left) $R_{\gamma}$ vs $p_{T}$ [111] for virtual photons (solid circles) [94] and real photons (open squares) [112] for minimum bias $\mathrm{Au}+\mathrm{Au}$ collisions at $\sqrt{s_{N N}}=200 \mathrm{GeV}$. b) (right) Ratio of direct- $\gamma$ $v_{2}$ to $\pi^{0} v_{2}$ for two reaction plane detectors [111.

$\geq 1.19 / 0.19 \approx 6$ [50], and allows the precision of $R_{\gamma}$ to be greatly improved as shown. Then, using the precise virtual photon $R_{\gamma}$ with the much higher statistics inclusive real- $\gamma$ data, one can derive $v_{2}$ for direct- $\gamma$ from the measured $v_{2}$ of inclusive real- $\gamma$ compared to the measured $v_{2}$ of $\gamma$ 's from $\pi^{0}$ and $\eta$ decay.

The result (Fig. $32 \mathrm{~b}$ ) [111] is that the $v_{2}$ of direct- $\gamma$ is large in the range $1 \leq p_{T} \leq 3 \mathrm{GeV} / \mathrm{c}$ but drops to zero for $p_{T} \geq 5 \mathrm{GeV} / \mathrm{c}$ where the photons are produced from initial hard-scattering and do not interact with the medium so that they do not flow. Since thermal radiation is produced in the flowing medium, the observed large $v_{2}$ in the range $1 \leq p_{T} \leq 3 \mathrm{GeV} / \mathrm{c}$, where the direct- $\gamma p_{T}$ spectrum is exponential (Fig. $24 \mathrm{p}$ ), confirms that these $\gamma$ are thermal radiation from the medium. What is very surprising is that the $v_{2}$ of the thermal photons is so large, the same or slightly greater than that of $\pi^{0}$ 's.

\section{A charming surprise}

One of the most exciting discoveries at RHIC, now confirmed at the LHC, is the suppression of heavy quarks comparable to that of $\pi^{0}$ from light quarks for $p_{T} \gtrsim 4 \mathrm{GeV} / \mathrm{c}$ as observed at RHIC using direct-single- $e^{ \pm}$from heavy quark $(c, b)$ decay (Fig. 33a) [113]. Also seen at RHIC is that heavy quarks exhibit collective flow $\left(v_{2}\right)$ (Fig. $33 \mathrm{~b}$ ), another indication of a very strong interaction with the medium.

At the LHC, ALICE [114] measured the suppression of reconstructed $D$ mesons containing $c$ quarks, and CMS [115] observed suppression of non-prompt $J / \Psi$ from b-quark decay (Fig. 34 a). The discovery at RHIC in 2007 was a total surprise and a problem since it appears to disfavor the radiative energy loss explanation [90, of suppression (also called jet-quenching) because heavy quarks should radiate much less than light quarks or gluons.

Many explanations have been offered including some from string theory; but the explanation I prefer was by Nino Zichichi [116] who proposed that since the standard model Higgs Boson, which gives mass to the Electro-Weak vector Bosons, does not necessarily give mass to Fermions, "it cannot be excluded that in a QCD coloured world (a QGP), the six quarks are all nearly massless". 

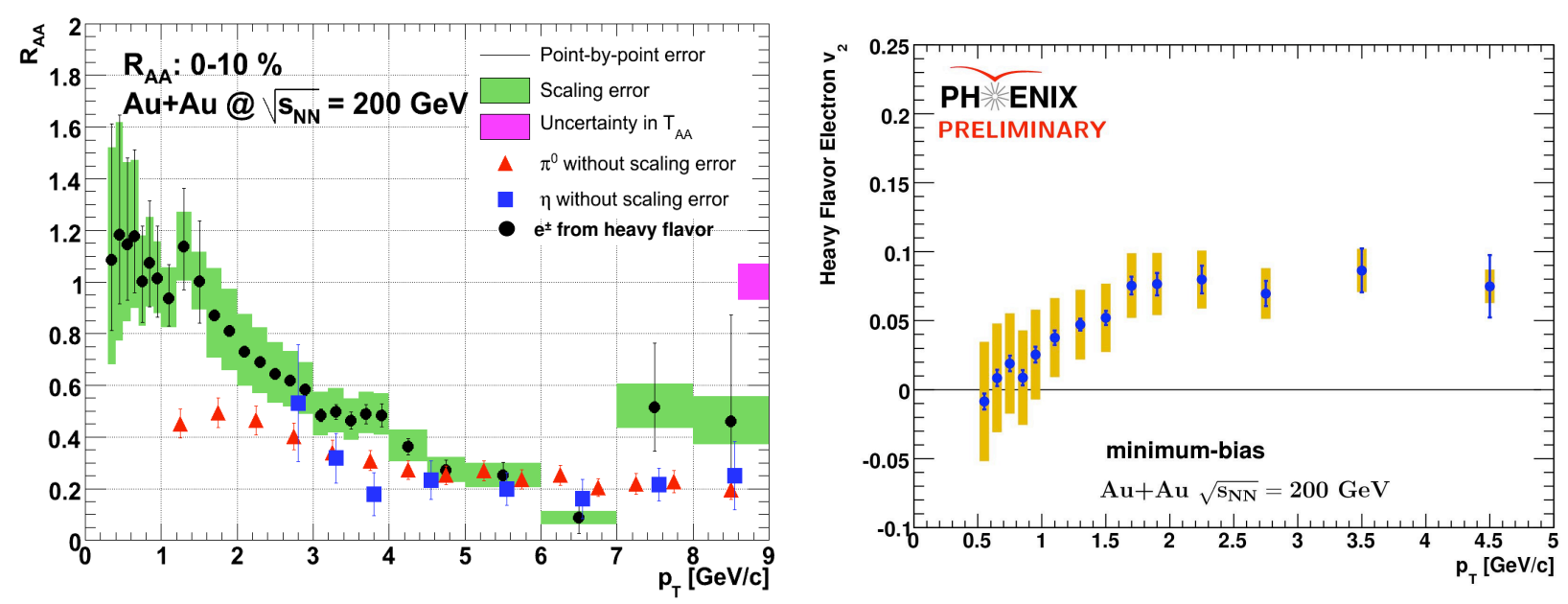

Figure 33: PHENIX [113] a) (left) $R_{A A}$ (central $\mathrm{Au}+\mathrm{Au}$ ) b) (right) $v_{2}$ (minimum bias $\mathrm{Au}+\mathrm{Au}$ ) as a function of $p_{T}$ for direct- $e^{ \pm}$at $\sqrt{s_{N N}}=200 \mathrm{GeV}$.
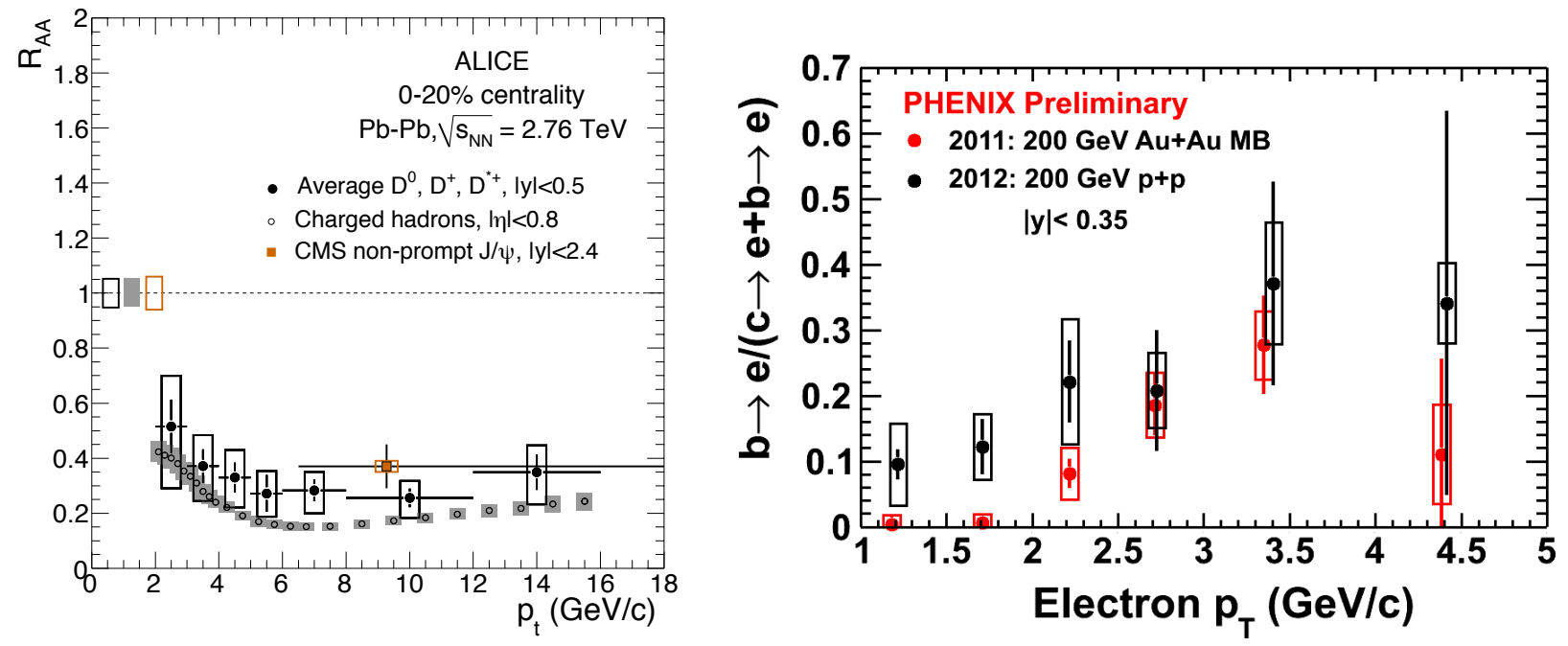

Figure 34: a) (left) $R_{A A}$ of ALICE [114] $D$-mesons, charged hadrons, and CMS [115] non-prompt $J / \Psi$, in central $(0-20 \%) \mathrm{Pb}+\mathrm{Pb}$ collisions at $\sqrt{s_{N N}}=2.76 \mathrm{TeV}$ b) (right) $b$-quark fraction $F_{b}=$ $b \rightarrow e /(c \rightarrow e+b \rightarrow e)$ of direct single- $e^{ \pm}$in $\mathrm{p}-\mathrm{p}$ and $\mathrm{Au}+\mathrm{Au}$ from PHENIX measurement of the Distance of Closest Approach (DCA) of the displaced vertex.

If this were true it would certainly explain why light and heavy quarks appear to exhibit the same radiative energy loss in the medium. This idea can, in fact, be tested because the energy loss of one hard-scattered parton relative to its partner, e.g. $g+g \rightarrow b+\bar{b}$, can be measured by experiments at RHIC and LHC using two-particle correlations in which both the outgoing $b$ and $\bar{b}$ are identified by measurement of the Distance of Closest Approach (DCA) of their displaced decay vertices in silicon vertex detectors. When such results are available, they can be compared to $\pi^{0}$-charged hadron correlations from light quark and gluon jets, for which measurement of the relative energy loss has been demonstrated at RHIC (recall Fig. 30] [109]. 
Of course, measurement of the Yukawa couplings to Fermions of the $125 \mathrm{GeV}$ Higgs Boson [117] at the LHC may be available in the not too distant future; but, already at Quark Matter 2012, the first direct measurement of $b$-quarks in p-p and Au+Au collisions at RHIC by their displaced vertices in the new PHENIX Silicon VTX detector was presented [118. Figure 34b shows the measurement of the $b$-quark fraction $F_{b}=b \rightarrow e /(c \rightarrow e+b \rightarrow e)$ of direct single $e^{ \pm}$in p-p and $\mathrm{Au}+\mathrm{Au}$ collisions at $\sqrt{s_{N N}}=200 \mathrm{GeV}$, using the PYTHIA $c$ and $b$ quark $p_{T}$ distributions in p-p collisions to calculate the DCA distributions of the $e^{ \pm}$in both p-p and $\mathrm{Au}+\mathrm{Au}$. The fact that the $\mathrm{Au}+\mathrm{Au}$ measurements for all $p_{T}^{e}$ are below the p-p measurements indicates clearly that the $b$-quark $p_{T}$ spectrum is modified in $\mathrm{Au}+\mathrm{Au}$ compared to $\mathrm{p}$-p. However, the correct conditional DCA distribution requires the actual (modified) b-quark $p_{T}$ spectrum in $\mathrm{Au}+\mathrm{Au}$, which must be obtained by iteration. Once the iteration has converged, the $R_{A A}^{b}\left(p_{T}^{e}\right)$ can be calculated from the measured $R_{A A}\left(p_{T}^{e}\right)$ of the direct-single- $e^{ \pm}$by the relation $R_{A A}^{b}\left(p_{T}^{e}\right)=R_{A A}\left(p_{T}^{e}\right) \times F_{b}^{A A} / F_{b}^{p p}$. For example, if the final $F_{b}^{A A}=F_{b}^{p p}$ then $R_{A A}^{b}\left(p_{T}^{e}\right)=R_{A A}\left(p_{T}^{e}\right)$.

\section{Do latest $J / \Psi$ results from ALICE prove deconfinement?}

In a previous article in this journal [13, I had noted that the dramatic difference in $\pi^{0}$ and $h^{ \pm}$ suppression from SpS to RHIC c.m. energy, $\sqrt{s_{N N}}=17.2$ to $200 \mathrm{GeV}$ (Fig. 27 a), is not reflected in $J / \Psi$ suppression, which is nearly identical at mid-rapidity at RHIC [119, 120] compared to the NA50 measurements at SpS 44]. (Fig. 35a). Coupled with the large, well-known, CNM effect on the $J / \Psi$ (recall Fig. 10), this reinforced my skepticism on the value of $J / \Psi$ suppression as a probe of deconfinement, in spite of the beauty and importance of the Matsui and Satz proposal [43.

The equality of $J / \Psi$ suppression at $\sqrt{s_{N N}}=17.2$ and $200 \mathrm{GeV}$ was was elegantly explained [121]
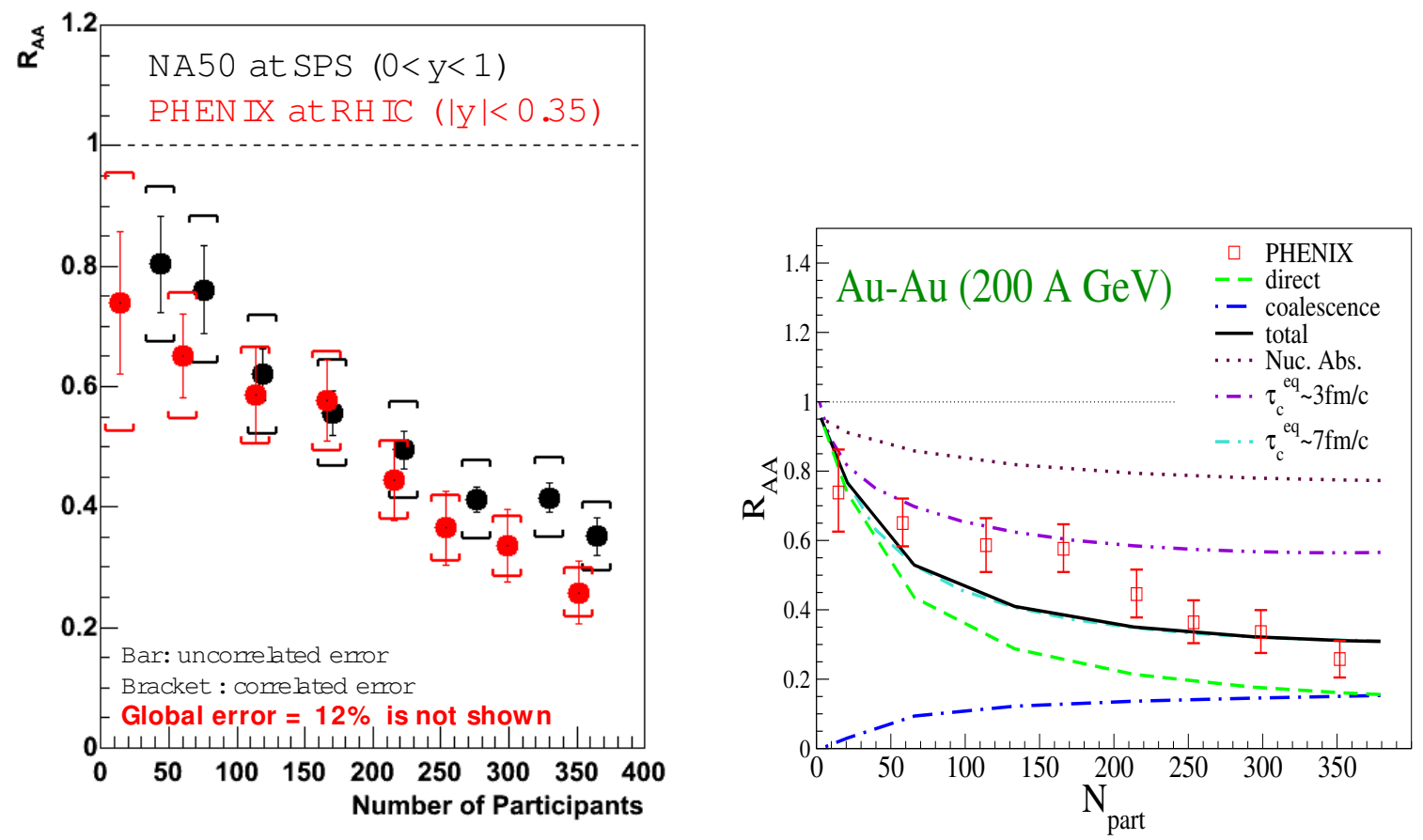

Figure 35: a) (left) $J / \Psi$ suppression relative to p-p collisions $\left(R_{A A}\right)$ as a function of centrality $\left(N_{\text {part }}\right)$ at RHIC [119, 120] and at the CERN/SPS [44. b) (right) PHENIX measurement of $R_{A A}$ as a function of centrality from (a) together with prediction from a coalescence model [121] 

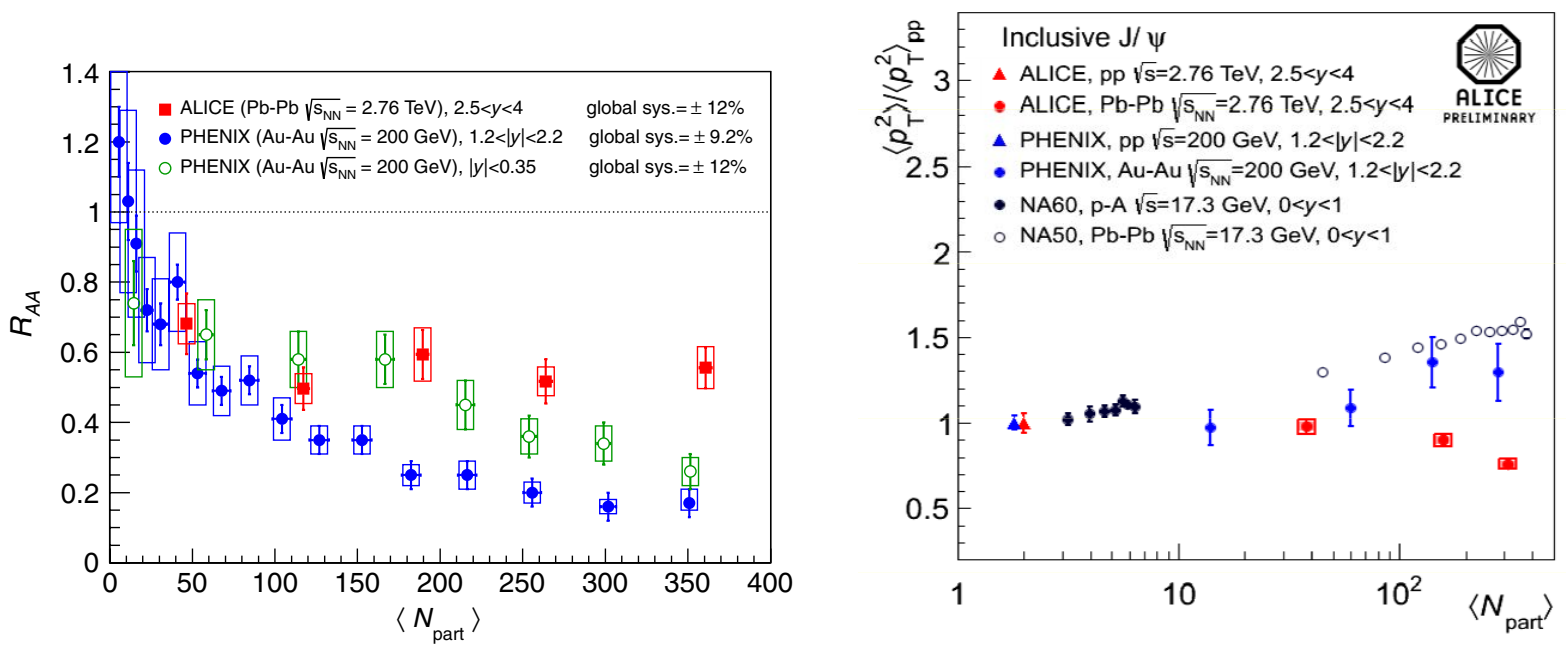

Figure 36: a) (left) $J / \Psi$ suppression relative to p-p collisions $\left(R_{A A}\right)$ as a function of centrality $\left(N_{\text {part }}\right)$ at RHIC [119] and at the CERN/LHC [125]. b) (right) ALICE measurement of $\left\langle p_{T}^{2}\right\rangle_{A A} /\left\langle p_{T}^{2}\right\rangle_{p p}$ for the $J / \Psi$ as a function of centrality compared to measurements at lower $\sqrt{s_{N N}}[126]$

as recombination or coalescence of $c$ and $\bar{c}$ quarks in the QGP to regenerate $J / \Psi$. Miraculously this made the observed $R_{A A}$ equal at SpS and RHIC c.m. energies (Fig. 35b) [120, 121. I called this my "Nightmare Scenario" because I thought that nobody would believe it. The good news was that such models are testable because they predict the reduction of $J / \Psi$ suppression or even an enhancement $\left(R_{A A}>1\right)$ at LHC energies [122, 123, 124], which would be spectacular, if observed.

In 2012, the ALICE experiment at LHC [125] presented a convincing measurement of reduced $J / \Psi$ suppression at $\sqrt{s_{N N}}=2.76 \mathrm{TeV}$ at forward rapidity compared to $\sqrt{s_{N N}}=200$ $\mathrm{GeV}$ (Fig. 36a) which, in my opinion, was clear proof the regeneration prediction. However, in 2013, Paolo Giubellino presented ALICE preliminary results [126] at this meeting for the ratio $\left\langle p_{T}^{2}\right\rangle_{A A} /\left\langle p_{T}^{2}\right\rangle_{p p}$ as a function of centrality in $\mathrm{Pb}+\mathrm{Pb}(\mathrm{Fig} 36 \mathrm{~b})$ which show a decrease from unity in p-p and mid-peripheral collisions to $\approx 0.7$ for central collisions while both the SpS and PHENIX data continue rising to values $\approx 1.2-1.5$. Paolo claimed that this proves deconfinement in central collisions.

In the discussion afterwards, I disagreed and claimed that the reduction of $\left\langle p_{T}^{2}\right\rangle_{A A} /\left\langle p_{T}^{2}\right\rangle_{p p}$ proves regeneration which is more probable at low $p_{T}$ [127]. Deconfinement would remove $J / \Psi$ at low $p_{T}$ in central collisions which would increase $\left\langle p_{T}^{2}\right\rangle_{A A} /\left\langle p_{T}^{2}\right\rangle_{p p}$ as shown by the PHENIX data. This is still good news for CERN because the clear observation of regeneration also proves directly the existence of the QGP at the LHC, since it is evidence that the $c$ and $\bar{c}$ quarks (with their color charge fully exposed) freely traverse the medium (with a large density of similarly exposed color charges) to find each other and form $J / \Psi$. Professor Zichichi uncharacteristically cut off the discussion of deconfinement due to time pressure and said that he agreed with Paolo.

After the session, Professor Tawfik pointed out to me that Helmut Satz had recently discussed a way to distinguish deconfinement in the presence of regeneration [128. The crucial issue is whether the medium modifies the fraction of produced $c-\bar{c}$ pairs which form $J / \Psi$. Dissociation of $J / \Psi$ in the medium would reduce the observed $J / \Psi /(c-\bar{c})$ ratio in A+A compared to p-p collisions, i.e. $R_{A A}^{J / \Psi} / R_{A A}^{c-\bar{c}} \ll 1$.

Satz [128] first compared $R_{A A}^{c-\bar{c}}$ to $R_{A A}^{J / \Psi}$ (Fig. 37) using LHC data from ALICE [114, 125] and 

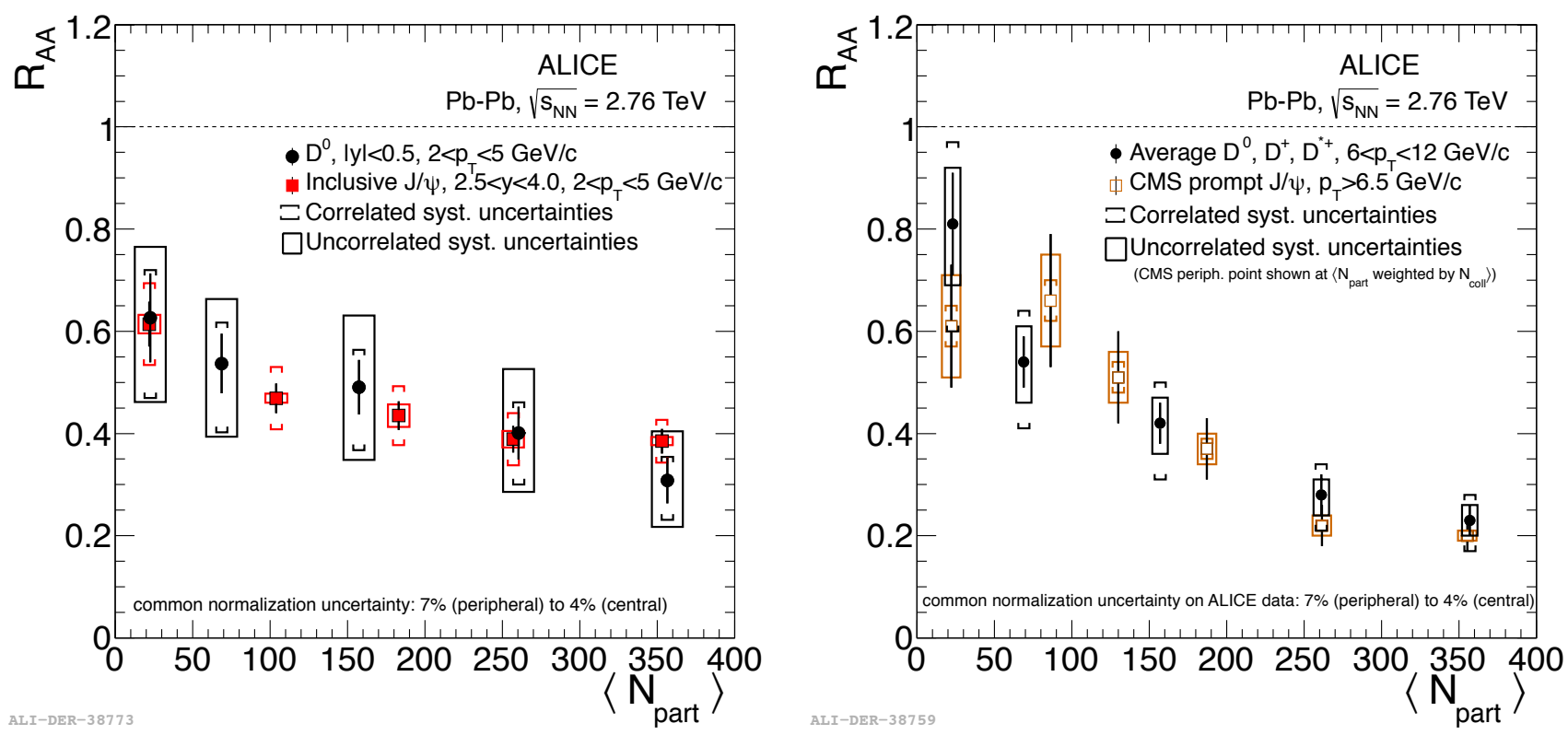

Figure 37: LHC data from ALICE [114, 125] and CMS [115], comparing $J / \Psi$ production to open charm production at intermediate (a) and higher (b) transverse momenta [128].
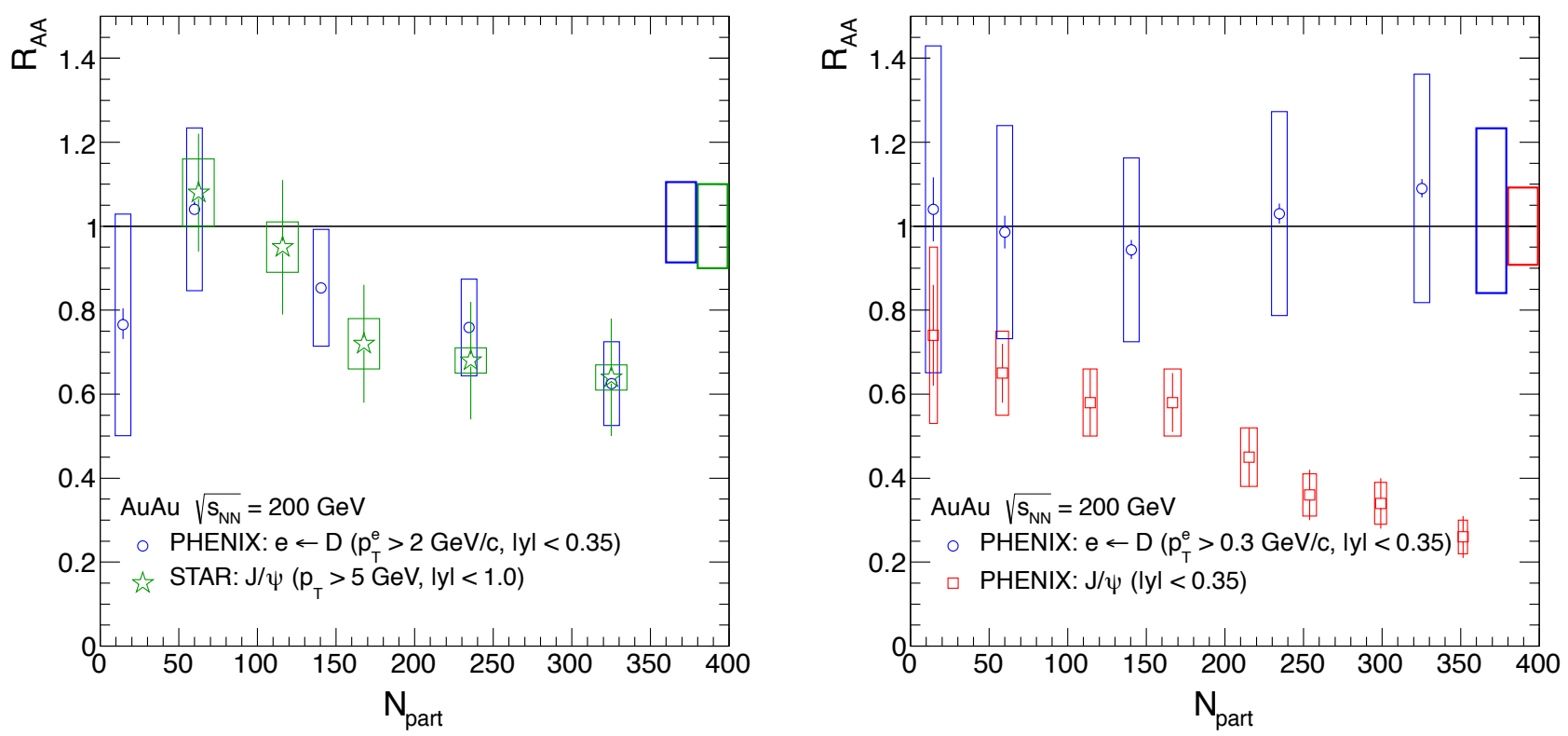

Figure 38: RHIC data from PHENIX [129, 119] and STAR [130], comparing $J / \Psi$ production to open charm production at high (a) and low (b) transverse momenta [128].

CMS [115] at intermediate $(2.5-4 \mathrm{GeV} / \mathrm{c})$ and higher $(6-12 \mathrm{GeV} / \mathrm{c}) p_{T}$. In both cases the $R_{A A}$ for open charm and $J / \Psi$ production show the same behavior within errors. "In other words, the reduction of the $J / \Psi$ is in complete agreement with that of open charm; there is neither suppression nor enhancement" [128]. However the conclusion is premature because the total $c-\bar{c}$ production and $R_{A A}$ at low $p_{T}$ was not yet available at LHC. 
Satz [128] contrasted this with the measurements from RHIC (Fig. 38) where at high $p_{T}$ the $R_{A A}$ of open charm and $J / \Psi$ track each other, as at LHC. However, at low $p_{T}$ "the $R_{A A}^{c-\bar{c}}$ is within errors unity over the entire centrality range; in contrast $R_{A A}^{J / \Psi}$ decreases strongly and thus here gives the correct $J / \Psi$ survival probability ... with increasing centrality, a smaller and smaller fraction of $c \bar{c}$ pairs go into $J / \Psi$ production, with a suppression of up to $75 \%$ for the most central collisions" [128].

I suppose this means that at RHIC there is evidence of deconfinement via "calibrated" $J / \Psi$ suppression [128], while for LHC the data are inconclusive until $R_{A A}$ of the total (i.e. low $p_{T}$ ) $c-\bar{c}$ production is available. Also, since new measurements of CNM effects at RHIC and LHC have produced surprising results in some cases, measurements of CNM effects in $J / \Psi$ and $c-\bar{c}$ production at both RHIC and LHC are needed for an unambiguous conclusion about whether $J / \Psi$ suppression is evidence for deconfinement via Debye screening in the QGP.

\section{Surprises in $\mathrm{d}+\mathrm{Au}$ and $\mathrm{p}+\mathrm{Pb}$ measurements}

\subsection{Hard-Scattering}

In 2013 , the major event was the $\mathrm{p}+\mathrm{Pb}$ run at $\mathrm{LHC}$ which also spurred new or improved $\mathrm{d}+\mathrm{Au}$ results from RHIC. Apart from one hard-scattering result to be presented first, all the results involve the predominant soft physics of multiplicity and $E_{T}$ distributions as well as flow. A new measurement of identified hadron production in both $\mathrm{Au}+\mathrm{Au} \mathrm{d}+\mathrm{Au}$ at $\sqrt{s_{N N}}=200 \mathrm{GeV}$ [131] gives some insight into the baryon anomaly. Figure 39 a shows $R_{A A}$ in $\mathrm{Au}+\mathrm{Au}$ for protons and
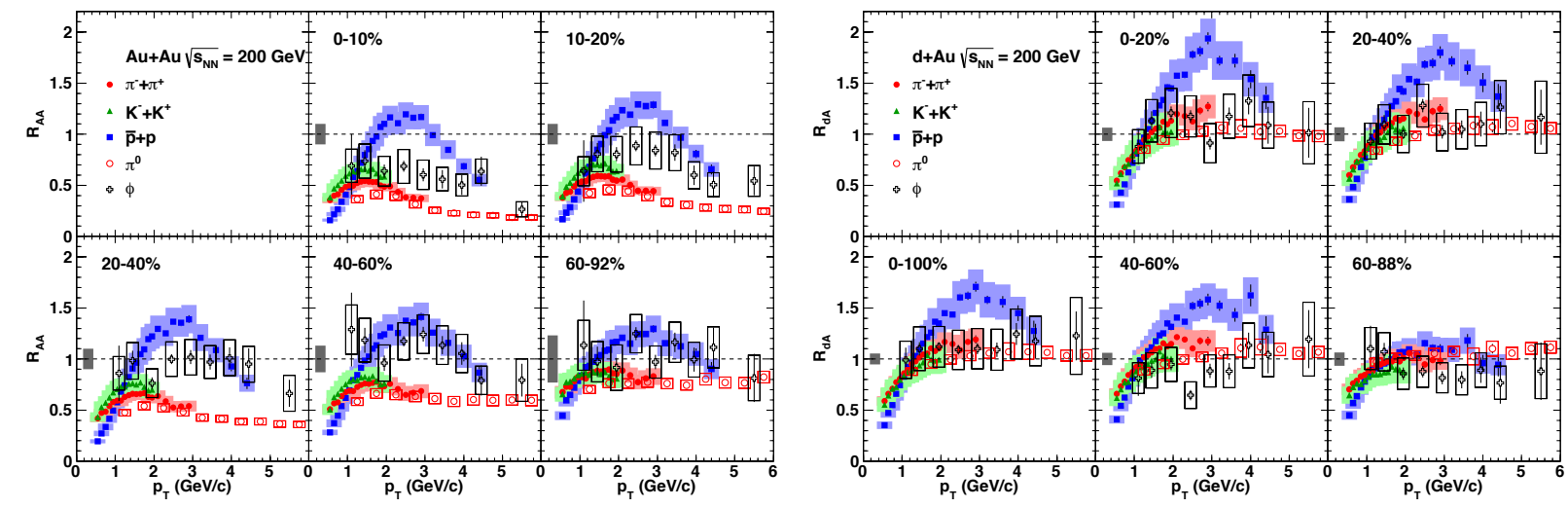

Figure 39: Measurements of $R_{A A}$ of identified particles as a function $p_{T}$ and centrality at $\sqrt{s_{N N}}=$ $200 \mathrm{GeV}$ [131]: a)(left) $\mathrm{Au}+\mathrm{Au}$; b) (right) d+Au.

mesons in the range $0.5<p_{T}<6.0 \mathrm{GeV} / \mathrm{c}$, where, in central collisions (0-10\%), all the mesons are suppressed for $p_{T}>2 \mathrm{GeV} / \mathrm{c}$ while the protons are enhanced for $2<p_{T}<4 \mathrm{GeV} / \mathrm{c}$ and then become suppressed at larger $p_{T}$. The $\mathrm{d}+\mathrm{Au}$ results in Fig. $39 \mathrm{~b}$ show no effect for the mesons, $R_{A A} \approx 1$ out to $p_{T}=6 \mathrm{GeV} / \mathrm{c}$; while the protons show a huge enhancement (Cronin effect) in all centralities except for the most peripheral (60-88\%). This suggests the need for a common explanation of the proton enhancement in both $\mathrm{Au}+\mathrm{Au}$ and $\mathrm{d}+\mathrm{Au}$ collisions, which is lacking at present. 


\subsection{Multiplicity and $E_{T}$ Distributions}

Returning to the soft physics of multiplicity and $E_{T}$ distributions, the PHOBOS experiment at RHIC, with a large pseudo-rapidity acceptance $-5<\eta<+5$ over the full azimuth had presented an instructive measurement of the charged multiplicity density, $d N_{\mathrm{ch}} / d \eta$ from the first $\mathrm{d}+\mathrm{Au}$ run in 2003 (Fig. 40a) 132. For peripheral collisions, the distribution is symmetric around mid-rapidity,
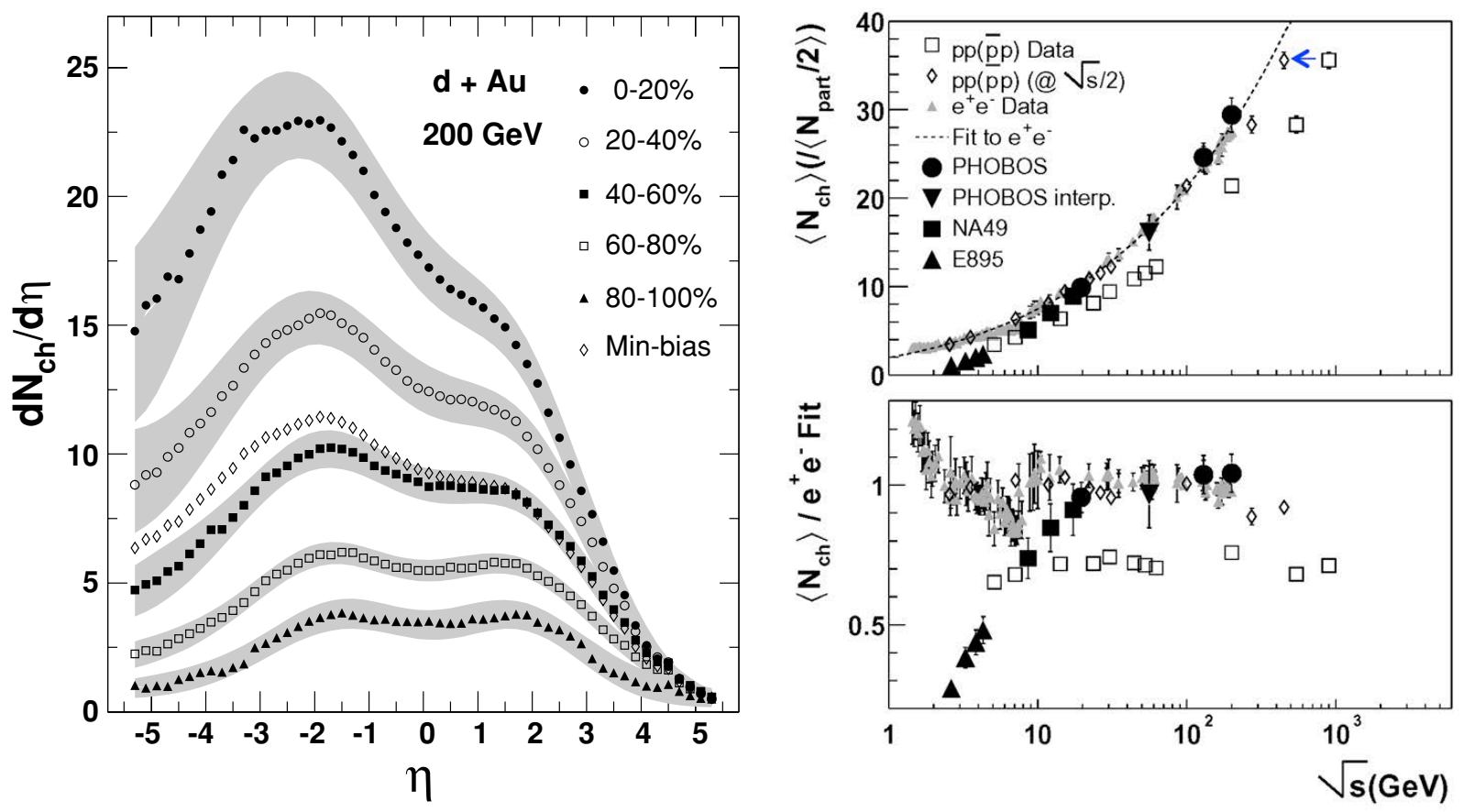

Figure 40: a) (left) Charged particle multiplicity density in rapidity, $d N_{\mathrm{ch}} / d \eta$, as a function of centrality in $\mathrm{d}+\mathrm{Au}$ collisions at $\sqrt{s_{N N}}=200 \mathrm{GeV}$ [132]. b) (right) Total charged multiplicity per nucleon pair in $\mathrm{p}-\mathrm{p}$ and $\mathrm{A}+\mathrm{A}$ collisions as a function of c.m. energy $\sqrt{s}$ compared to $e^{+}+e^{-}$ collisions [134].

as in p-p collisions. However, with increasing centrality, the multiplicity increases over the whole $\eta$ range but with a larger increase at negative $\eta$ (the Au rapidity) such that the peak of the distribution steadily shifts in the direction of the Au nucleus.

These features which are similar to what was first observed in fixed target $\mathrm{p}+\mathrm{A}$ experiments at $\sqrt{s_{N N}} \sim 19.4 \mathrm{GeV}$ could be explained (c. 1976) by a simple model, the Wounded Nucleon Model (WNM) [133. From relativity and quantum mechanics the only thing that can happen to a relativistic nucleon when it interacts with another nucleon in a nucleus is to become an excited nucleon with the same energy but reduced longitudinal momentum (rapidity). It remains in that state inside the nucleus because the uncertainty principle and time dilation prevent it from fragmenting into particles until it is well outside the nucleus. If one makes the further assumptions that an excited nucleon interacts with the same cross section as an unexcited nucleon and that the successive collisions of the excited nucleon do not affect the excited state or its eventual fragmentation products, this leads to the conclusion that the elementary process for particle production in nuclear collisions is the excited nucleon, and to the prediction that the multiplicity in nuclear interactions should be proportional to the total number of projectile and target participants (Wounded Nucleons) [133, rather than to the total number of collisions.

Another interesting effect observed by PHOBOS [134] is that the "leading particle effect" in p-p collisions, as discovered by Zichichi and collaborators [135], in which the total multiplicity at c.m. 

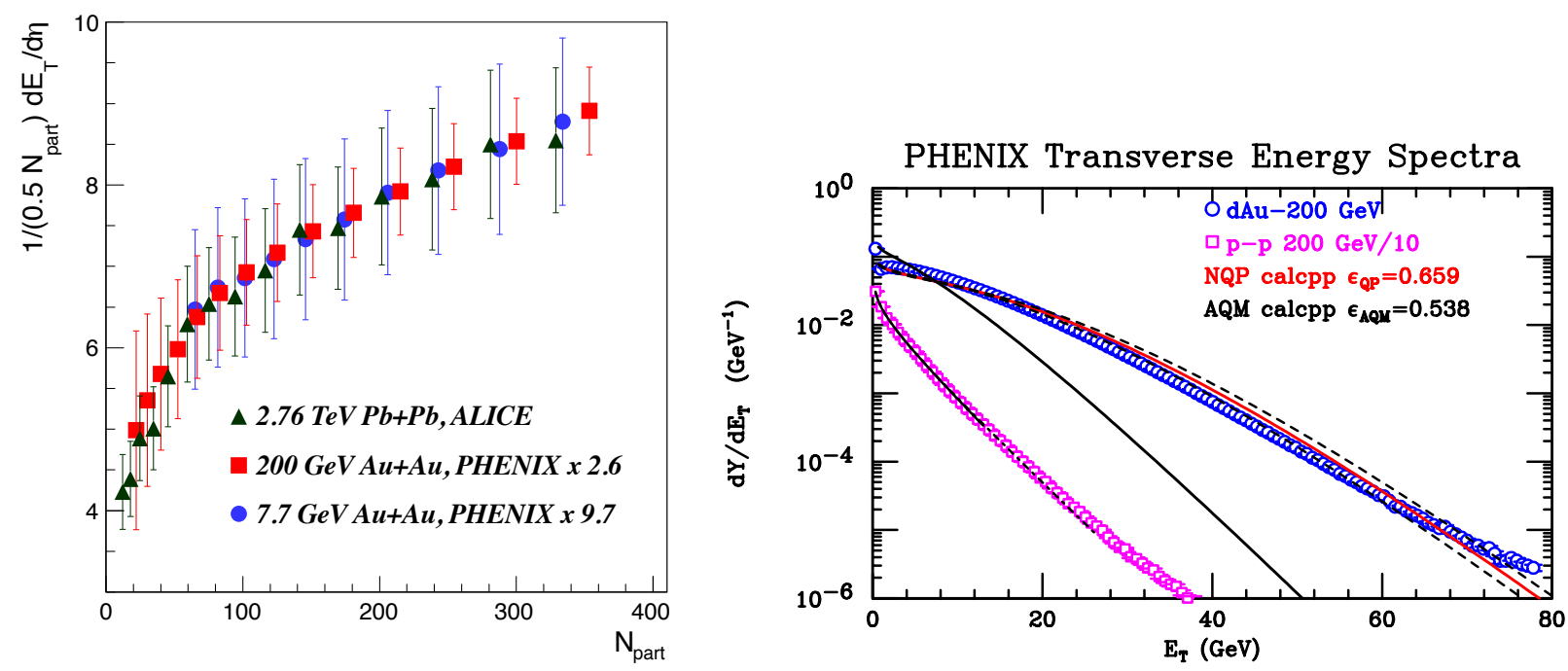

Figure 41: a) (left) $d E_{T}^{A A} / d \eta /\left(0.5\left\langle N_{\text {part }}\right\rangle\right)$ vs. $\left\langle N_{\text {part }}\right\rangle$ in $\mathrm{Au}+\mathrm{Au}$ and $\mathrm{Pb}+\mathrm{Pb}$ collisions from $\sqrt{s_{N N}}=0.0077$ to $2.76 \mathrm{TeV}$. b) (right) PHENIX measurement [136] of $E_{T}$ distributions for $\mathrm{p}+\mathrm{p}$ and $\mathrm{d}+\mathrm{Au}$ at $\sqrt{s_{N N}}=200 \mathrm{GeV}$ with calculations of the $\mathrm{d}+\mathrm{Au}$ spectrum based on the AQM (color-strings) and the number of constituent-quark participants (NQP).

energy $\sqrt{s_{\mathrm{pp}}}$ is equal to that in $e^{+} e^{-}$collisions at $\sqrt{s_{\mathrm{ee}}}=\sqrt{s_{\mathrm{pp}}} / 2$ (the "effective energy") because the leading protons carry away half the p-p c.m. energy, is absent in A+A collisions (Fig. 40p). This observation seems to contradict the WNM, in which the key assumption is that what counts is whether or not a nucleon was struck, not how many times it was struck.

In fact, the WNM fails badly at mid-rapidity for both $d N_{c h} / d \eta$ and $d E_{T} / d \eta$ as shown by a plot of $d E_{T} / d \eta /\left(N_{\text {part }} / 2\right)$ vs. $N_{\text {part }}$ from PHENIX which should be constant if the WNM were true (Fig. 41a). The fact that the scaled evolution with centrality is the same from $\sqrt{s_{N N}}=7.7 \mathrm{GeV}$ to $2.76 \mathrm{TeV}$ indicates that the dominant effect is the nuclear geometry of the $\mathrm{A}+\mathrm{A}$ collision. It has been shown that the evolution in Fig. 41 a can be explained by a nuclear geometry based on the number of constituent-quark participants, the NQP model [137, 138. Thus the shape of the data in Fig. 41 is imply the number of constituent-quark participants/nucleon participant, $N_{\mathrm{qp}} / N_{\text {part }}$.

For symmetric systems such as $\mathrm{Au}+\mathrm{Au}$, the NQP model is identical to another model from the 1970's, the Additive Quark Model (AQM) [139]. The AQM is actually a model of particle production by color-strings in which only one color-string can be attached to a constituent-quark participant. Thus, for asymmetric systems such as $d+A u$, the maximum number of color-strings is limited to the number of constituent-quarks in the lighter nucleus, or six for $\mathrm{d}+\mathrm{Au}$, while the NQP allows all the quark participants in both nuclei to emit particles. A new PHENIX measurement (Fig. 41b) [136] shows that the NQP model gives the correct $E_{T}$ distribution in d+Au, while the AQM has a factor of 1.7 less $E_{T}$ emission due to the restriction on the number of effective constituent-quarks in the larger nucleus.

The Wounded Nucleon (WNM), Additive Auark (AQM) and constituent-Quark-Participant (NQP) models are all examples of extreme-independent models in which the nuclear geometry of the interaction can be calculated in independently of the dynamics of particle production, which can be derived from experimental measurements, usually the $p+p$ (or $p+A$ ) measurement in the same detector. The constituent-quark method for $E_{T}$ distributions [136] is illustrated in Fig. 42. The p-p $E_{T}$ distribution (Fig. 42 a) is calculated as the properly weighted sum of 2 to 6 convolutions of the fitted $E_{T}$ distribution of a constituent-quark-participant, which is then applied to d+Au (Fig. 41b) 

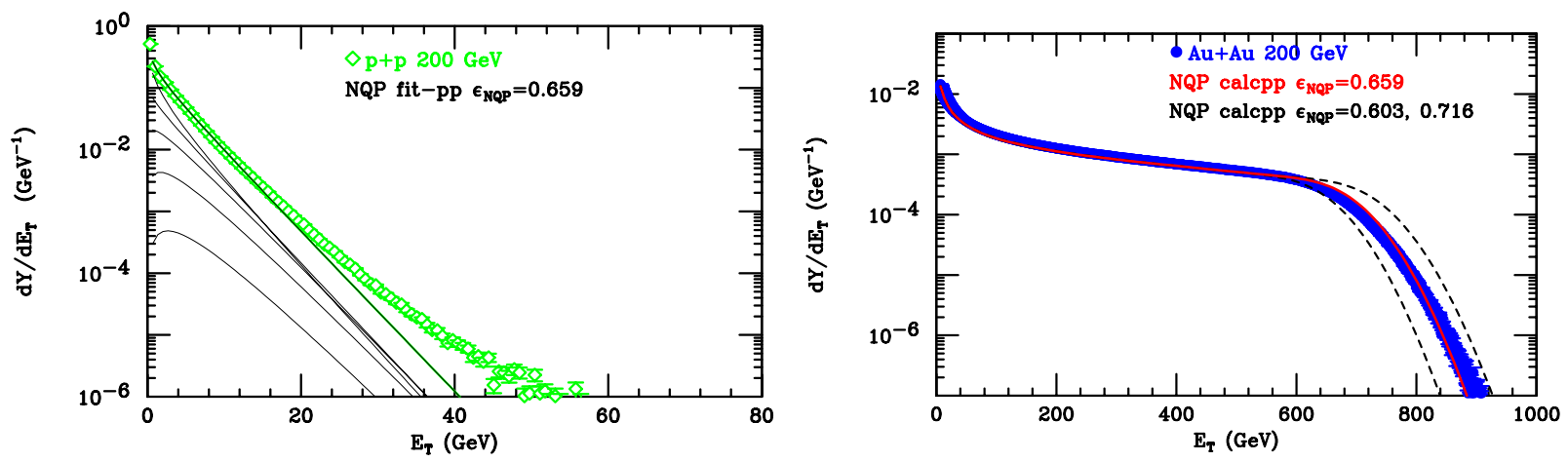

Figure 42: PHENIX [136] $E_{T}$ distribution in $\sqrt{s_{N N}}=200 \mathrm{GeV}$ p-p and $\mathrm{Au}+\mathrm{Au}$ collisions. a) (left) Fit of p-p distribution to a sum of properly weighted $2,3, \ldots 6$ constituent-quark-participant $E_{T}$ distributions. Lines indicate the individual distributions plus the sum. The $q-q$ cross section had been tuned to reproduce the inelastic p-p cross section. b) (right) Calculation of the $\mathrm{Au}+\mathrm{Au}$ $E_{T}$ distribution in the NQP model as the sum of properly weighted convolutions of the constituentquark-participant $E_{T}$ distribution derived in (a). Dashed lines indicate the systematic uncertainty.

and $\mathrm{Au}+\mathrm{Au}$ collisions (Fig. 42 b), with excellent agreement in both cases. In a standard Monte Carlo Glauber calculation of the nuclear geometry, the positions of the three-constituent quarks are generated about the position of each generated nucleon according to the measured charge distribution of the proton, which gives a physical basis for "proton size fluctuations" discussed at LHC [140].

Constituent-quark-participants might also explain the increase of the "effective energy" in $\mathrm{A}+\mathrm{A}$ collisions compared to p-p collisions discussed previously (Fig. 40 p). The $\left\langle N_{\mathrm{qp}} / N_{\mathrm{part}}\right\rangle$ is 1.5 for a p-p collision but rises to 2.3-2.7 for more central (0-50\%) A+A collisions. Thus the "effective energy" for particle production increases due to an increase in the number of (constituent-quark) participants, not because of additional collisions of a given participant. This preserves the assumption in these "extreme-independent" participant models that successive collisions of a participant do not increase its particle emission.

\subsection{Collective Flow in $\mathrm{d}+\mathrm{Au}($ and $\mathrm{p}+\mathrm{Pb}$ ) collisions?}

The most surprising soft-physics result in $\mathrm{p}+\mathrm{Pb}$ and $\mathrm{d}+\mathrm{Au}$ physics in 2013 concerned what looks very much like collective flow observed in these small systems, where no (or negligible) medium or collective effect was expected. Fig. 43 a shows a LEGO plot of $\Delta \eta, \Delta \phi$, the difference in polar and azimuthal angles from correlations of two particles with $1<p_{T}<3 \mathrm{GeV} / \mathrm{c}$ in $\mathrm{p}+\mathrm{Pb}$ by $\mathrm{CMS}$ at $\sqrt{s_{N N}}=5.02 \mathrm{TeV}$ [141]. A clear $1+2 v_{2} \cos 2 \Delta \phi$ modulation of the distribution independent of $\Delta \eta$ is observed, called the 'ridge' in $\mathrm{Au}+\mathrm{Au}$ collisions where the modulations $v_{2}, v_{3}, \ldots v_{n}$ are attributed to collective flow of the QGP medium. At RHIC, PHENIX confirmed this result in $\mathrm{d}+\mathrm{Au}$ collisions at $\sqrt{s_{N N}}=200 \mathrm{GeV}$ (Fig. $43 \mathrm{~b}$ ) [142]. In order to remove any $v_{2}$ effect due to two-particle correlations of hard-scattering, lower $p_{T}$ triggers were used as well as cuts in $\Delta \eta$ to remove the same-side peak. Also, since there is no suppression of hard-scattering in $\mathrm{p}+\mathrm{Pb}$ or $\mathrm{d}+\mathrm{Au}$ collisions (recall Fig. 39), the conditional two-particle yield from di-jets is independent of centrality. Thus, any residual hard-scattering effect was removed by subtracting the peripheral (50-88\%) from the central (0-5\%) measurement which revealed the beautiful $\cos 2 \Delta \phi$ curve characteristic of elliptical flow shown in Fig. 43 b.

Figure 44 compares the $v_{2}$ measurements vs $p_{T}$ from $\mathrm{d}+\mathrm{Au}$ at $\sqrt{s_{N N}}=200 \mathrm{GeV}$ and $\mathrm{p}+\mathrm{Pb}$ at 

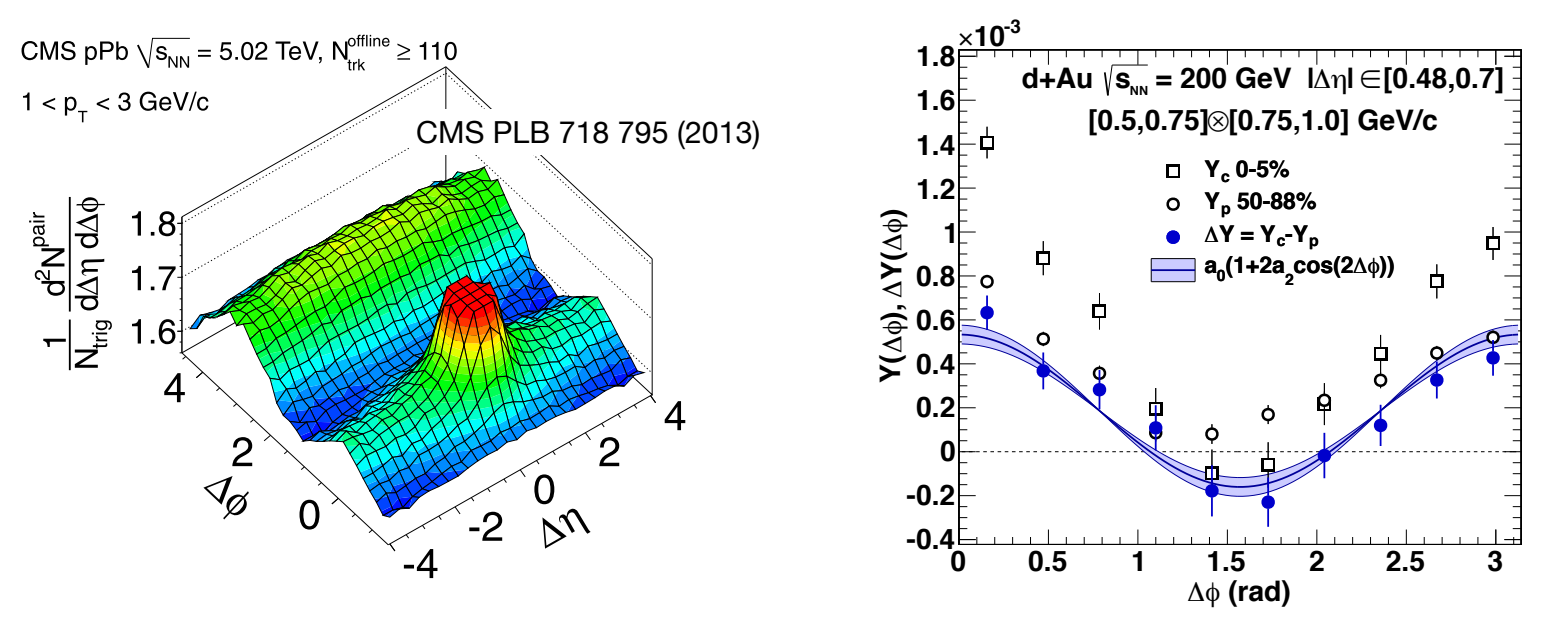

Figure 43: a) (left) CMS ridge in two-particle correlations [141]. b) (right) PHENIX two-particle azimuthal correlations in $\mathrm{d}+\mathrm{Au}$ at RHIC [142].
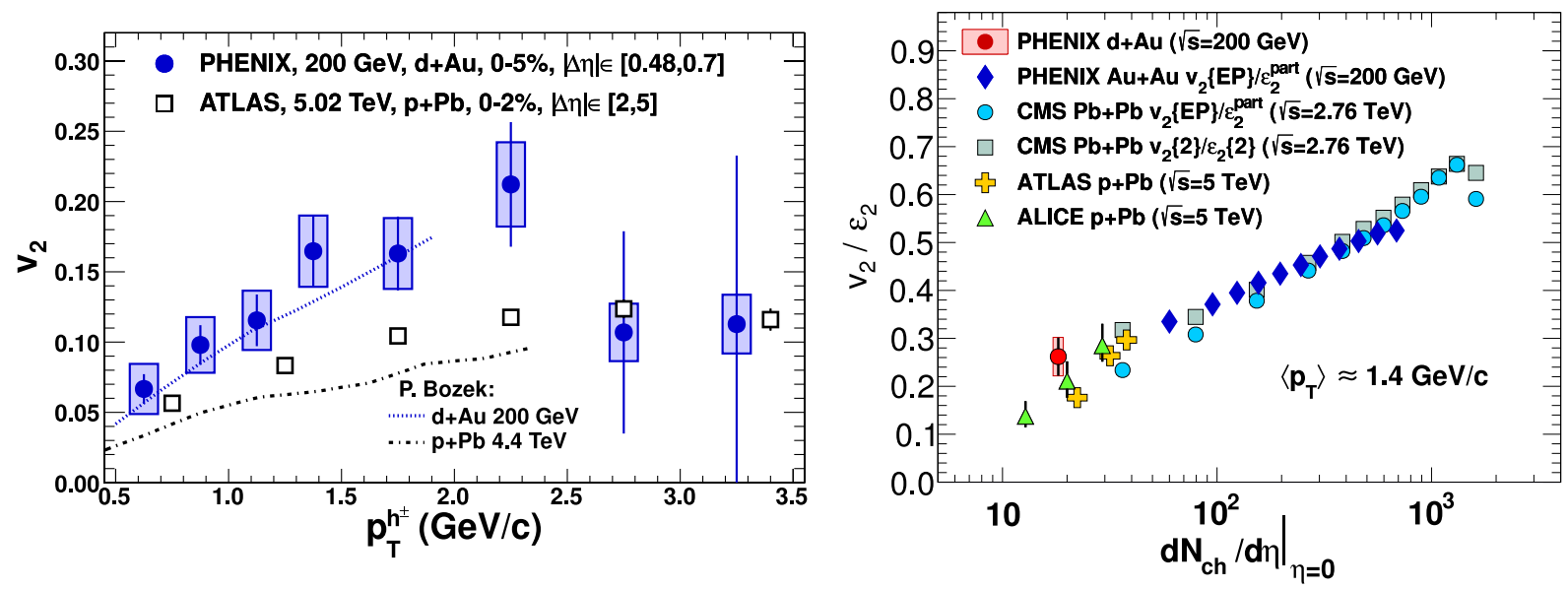

Figure 44: a) (left) Comparison of $v_{2}$ vs. $p_{T}$ in $\mathrm{d}+\mathrm{Au}$ at RHIC to $\mathrm{p}+\mathrm{Pb}$ at LHC [142]. b) (right) Compilation of $v_{2} / \varepsilon$ vs. $d N_{\mathrm{ch}} / d \eta$ at $p_{T}=1.4 \mathrm{GeV} / \mathrm{c}$ in $\mathrm{d}+\mathrm{Au}, \mathrm{p}+\mathrm{Pb}, \mathrm{Au}+\mathrm{Au}$ and $\mathrm{Pb}+\mathrm{Pb}$ collisions at RHIC and LHC [142].

$5.02 \mathrm{TeV}$. The larger values from the $\mathrm{d}+\mathrm{Au}$ results are thought to be due to the larger eccentricity $(\varepsilon)$ of the two-nucleon deuteron compared to the single nucleon proton. In fact, the values of $v_{2} / \varepsilon$ from $\mathrm{d}+\mathrm{Au}$ and $\mathrm{p}+\mathrm{Pb}$ are consistent with the dependence of $v_{2} / \varepsilon$ on $d N_{\mathrm{ch}} / d \eta$ (Fig. 44 $\mathrm{b}$ ) observed in $\mathrm{Au}+\mathrm{Au}$ and $\mathrm{Pb}+\mathrm{Pb}$ collisions, which was taken as proof of collective flow from hydrodynamics. These new results again underscore the importance of $\mathrm{p}-\mathrm{p}$ and $\mathrm{p}+\mathrm{A}$ comparison data to understand the observations in A+A collisions, where the detailed physics of the QGP is far from understood.

\section{The Future}

Toward this goal, PHENIX has proposed a new more conventional collider detector, s(uper)PHENIX, based on a thin-coil superconducting solenoid, to concentrate on hard-scattering and jets. This would replace the very successful but 15 year old special purpose small aperture two-arm spectrometer designed to measure $J / \Psi \rightarrow e^{+}+e^{-}$down to zero $p_{T}$ at mid-rapidity, the original expected signal for deconfinement, as well as identified particles such as single $e^{ \pm}$from heavy quark 


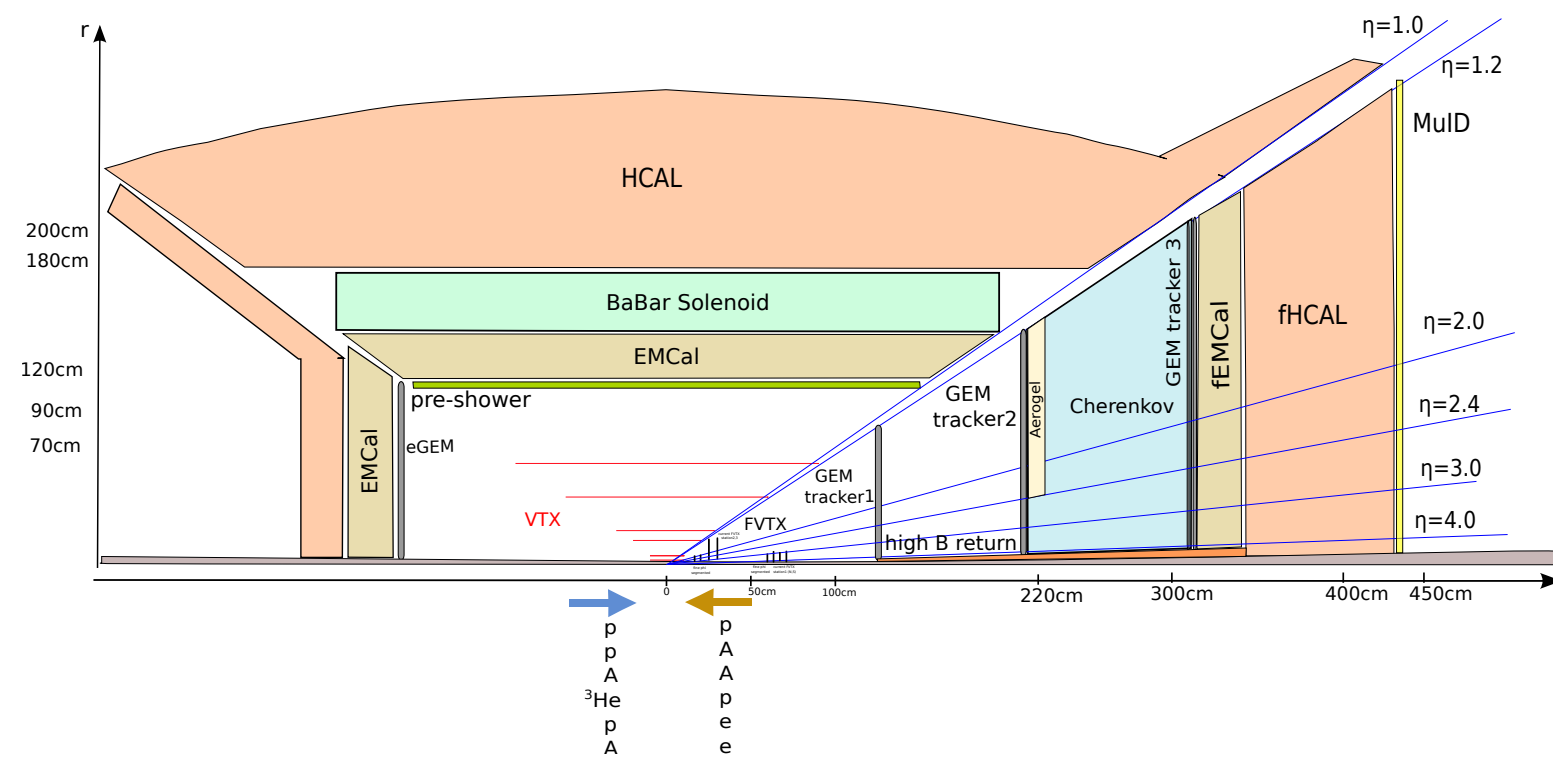

Figure 45: a) sPHENIX concept with forward detector [143].

decay, $\pi^{0}, \eta$ and other hadrons (recall Fig. $24 \mathrm{~b}$ ) that could cause background to the $J / \Psi$ but which turned out to be valuable probes of the QGP. This year sPHENIX got a big boost by acquiring the (made in Italy) BABAR solenoid magnet from SLAC which became available when the B-factory in Italy was unfortunately cancelled. Conceptual design of the new experiment is well underway, with mid-rapidity, forward and eRHIC capability [143] (Fig. 45]. New collaborators would be most welcome.

\section{References}

[1] Report of the Workshop on BeV/Nucleon Coliisions of Heavy Ions-How and Why, Bear Mountain, NY, 29 November-1 December 1974. (BNL-50445, Upton NY, 1975).

[2] See Ref. [3] for a more extensive list of references.

[3] M. J. Tannenbaum, Rep. Prog. Phys. 69 (2006) 2005-2059.

[4] E. V. Shuryak, Phys. Repts. 61 (1980) 71-158.

[5] BRAHMS Collab. (I. Arsene et al.), Nucl. Phys. A757 (2005) 1-27.

[6] PHOBOs Collab. (B. B. Back et al.), Nucl. Phys. A757 (2005) 28-101.

[7] STAR Collab. (J. Adams et al.), Nucl. Phys. A757 (2005) 102-183.

[8] PHENIX Collab. (K. Adcox et al.), Nucl. Phys. A757 (2005) 184-283.

[9] D. Rischke and G. Levin, eds., Nucl. Phys. A750 (2005) 1-171.

[10] Press Conference, A New State of Matter Created at CERN http://newstatematter.web.cern.ch/newstate-matter/Story.html

[11] New York Times, February 10, 2000, Page 1, Particle Physicists Getting Closer To the Bang That Started It All. 
[12] Discoveries and methods at RHIC have also been discussed by the present author in ISSP proceedings for the years 2008 (arXiv:0906.0745), 2009 (Ref. [13]) as well as in a recent textbook (Ref. [14]).

[13] M. J. Tannenbaum, Int. J. Mod. Phys. A26 (2011) 5299-5335.

[14] Jan Rak and Michael J. Tannenbaum, High $p_{T}$ Physics in the Heavy Ion Era, (Cambridge Univ. Press, Cambridge \& New York, 2013).

[15] A. Aad, et al. (ATLAS Collab.), Phys. Rev. Lett. 105 (2010) 252303.

[16] S. Chatrchyan, et al. (CMS Collab.), Phys. Rev. C84 (2011) 024906.

[17] M. Harrison, T. Ludlam and S. Qzaki (eds.), The Relativistic Heavy Ion Collider Project: RHIC and its Detectors, Nucl. Instrum. Methods A499 (2003) 235-880.

[18] NASA/BNL Space Radiation Program, http://www.bnl.gov/medical/NASA/LTSF.asp

[19] G. Bunce, et al., Ann. Rev. Nucl. Part. Sci. 50 (2000) 525.

[20] M. Blaskiewicz and W. Fischer, CERN Courier 52 (Oct. 2012) 17.

[21] E. J. Bleser, et al., Nucl. Instrum. Methods A235 (1985) 435.

[22] R. M. Bozorth, Ferromagnetism (VanNostrand, New York, 1951)

[23] ALPHA Collab. (G. B. Andresen et al.), Nature Physics 7 (2011) 558-564; Nature 468 (2010) 673-676.

[24] Press Release, "Indication of Electron Neutrino Appearance at the T2K Experiment" June 15, 2011, http://www.kek.jp/intra-e/press/2011/J-PARC_T2Kneutrino.html

[25] R. Chasman, G. K. Green and E. M. Rowe, IEEE Trans Nucl. Sci. NS-22 (1975) 1765-1767.

[26] NSLS-II Source Properties and Floor Layout http://www.bnl.gov/ps/docs/pdf/ SourceProperties.pdf

[27] G. W. Bennett, et al., Phys. Rev. D73 (2006) 072003.

[28] F. J. M. Farley, J. Bailey, R. C. A. Brown, M. Giesch. H. Jostlein, S. van der Meer, E. Picasso and M. Tannenbaum, Nuovo Cimento 45A (1966) 281-286.

[29] G. Charpak, F. J. M. Farley, R. L. Garwin, T. Muller, J. C. Sens and A. Zichichi, Nuovo Cimento 37 (1965) 1241-1363.

[30] R. W. Ellsworth, A. C. Melissinos, J. H. Tinlot, H. von Briesen, Jr., T. Yamanouchi, L. M. Lederman, M. J. Tannenbaum, R. L. Cool and A. Maschke, Phys. Rev. 165 (1968) 1449-1465.

[31] G. Danby, J-M. Gaillard, K. Goulianos, L. M. Lederman, N. Mistry, M. Schwartz and J. Steinberger, Phys. Rev. Lett. 9 (1962) 36-44.

[32] J. H. Christenson, J. W. Cronin, V. L. Fitch and R. Turlay, Phys. Rev. Lett. 13 (1964) 138-140.

[33] CCR Collab. (R. L. Cool et al.), in Proc. XVI Int. Conf. High Energy Physics, eds. J. D. Jackson and A. Roberts (NAL, Batavia, IL, 1973), Vol 3, p. 317. 
[34] J. F. Owens and J. D. Kimel, Phys. Rev. D18 (1978) 3313-3319.

[35] R. P. Feynman, R. D. Field, and G. C. Fox, Phys. Rev. D18 (1978) 3320-3343.

[36] C. De Marzo, et al., Phys. Lett. B112 (1982) 173-177.

[37] J. D. Bjorken, Phys. Rev. D8 (1973) 4098-4106.

[38] C. Bromberg, et al., Nucl. Phys. B134 (1978) 189-241.

[39] UA1 Collab. (G. Arnison et al.), Transverse Energy Distributions in the Central Calorimeters (1982), CERN-EP-82-122.

[40] UA2 Collab. (J.-P. Repellin et al.), in Proc. 21st Int. Conf. High Energy Physics, eds P. Petiau and M. Porneuf (Journal de Physique Colloques, Paris, 1982), vol. 43, pp. C3-571-C3-578.

[41] UA1 Collab. (G. Arnison et al.), Phys. Lett. B107 (1981) 320-324.

[42] Particle Data Group (C. Amsler et al.), Phys. Lett. B667 (2008) 1.

[43] T. Matsui and H. Satz, Phys. Lett. B178 (1986) 416.

[44] NA50 Collab. (B. Alessandro et al.), Eur. Phys. J. C39 (2005) 335-345. See also, F. Prino, Proc. XXX International Symposium on Multiparticle Dynamics, arXiv:hep-ex/0101052v1.

[45] E772 Collab. (D. M. Alde et al.), Phys. Rev. Lett. 66 (1991) 2285-2288. See, also, M. J. Leitch, Eur. Phys. J. C43 (2005) 157-160 and references therein.

[46] A. Breskin and R. Voss (eds.), The Large Hadron Collider: Accelerator and Experiments, J. Instrum. 3 (2008) S08001-S08007.

[47] PHENIX Collab. (J. T. Mitchell et al.), Nucl. Phys. A904,905 (2013) 903c.

[48] PHENIX Collab. (S. S. Adler et al.), Phys. Rev. C71 (2005) 034908.

[49] ALICE Collab. (K. Aamodt et al.), Phys. Rev. Lett. 106 (2011) 032301.

[50] See, for example, Refs. [13, 14].

[51] The detector is so non-conventional that it made the cover of Physics Today, October 2003.

[52] STAR Collab. (H. Agakishiev et al.), Nature 473 (2011) 353-356.

[53] STAR Collab. (B. I. Abelev, et al.), arXiv:0909.0566v1 [nucl-ex],

[54] R. A. Lacey, Nucl. Phys. A774 (2006) 199-214.

[55] PHENIX Collab. (M. Kaneta et al.), J. Phys. G30 (2004) S1217-S1220.

[56] PHENIX Collab. (A. Adare et al.), Phys. Rev. Lett. 98 (2007) 162301.

[57] J.-Y. Ollitrault, Phys. Rev. D46 (1992) 229-245; Nucl. Phys. A638 (1998) 195c-206c.

[58] H. Heiselberg and A.-M. Levy, Phys. Rev. C59 (1999) 2716-2727.

[59] S. A. Voloshin, Nucl. Phys. A715 (2003) 379c-388c. 
[60] D. Teaney, Phys. Rev. C68 (2003) 034913.

[61] P. K. Kovtun, D. T. Son, and A. O. Starinets, Phys. Rev. Lett. 94 (2005) 111601.

[62] R. A. Lacey, et al., Phys. Rev. Lett. 98 (2007) 092301.

[63] L. P. Csernai, J. I. Kapusta, and L. D. McLerran, Phys. Rev. Lett. 97 (2006) 152303.

[64] PHENIX Collab. (A. Adare et al.), Phys. Rev. C78 (2008) 014901; Phys. Rev. C77 (2008) 011901(R).

[65] PHENIX Collab. (A. Adare et al.), Phys. Rev. Lett. 98 (2007) 232302.

[66] J. Casalderrey-Solana, E. V. Shuryak and D. Teaney, J. Phys: Conf. Series 27 (2005) 22-31.

[67] For example, see Ref. 64] for a discussion and list of references.

[68] PHENIX Collab. (J. T. Mitchell et al.), J. Phys. G34 (2007) S911-S914.

[69] STAR Collab. (B. I. Abelev et al.), Phys. Rev. C80 (2009) 064912.

[70] STAR Collab. (J. Putschke et al.), Nucl. Phys. A783 (2007) 507c-510c.

[71] P. Jacobs, Eur. Phys. J. C43 (2005) 467-473.

[72] B. H. Alver, C. Gombeaud, M. Luzum, and J. Y. Ollitrault, Phys. Rev. C82 (2010) 034913.

[73] B. Alver and G. Roland, Phys. Rev. C81 (2010) 054905.

[74] J. Takahashi, et al., Phys. Rev. Lett. 103 (2009) 242301. Also, see A. P. Mishra, et al., Phys. Rev. C77 (2008) 064902.

[75] PHENIX Collab. (S. Esumi, et al.), arXiv:1110.3223v1 [nucl-ex].

[76] Presentation by M. Gaździcki at Quark Matter 2011, J. Phys. G38 (2011) 124024. Also see NA49 Collab. (M. Gaździcki et al.), J. Phys. G30 (2004) S701-S708.

[77] STAR collab. (L. Kumar et al.), J. Phys. G38 (2011) 124145, (B. Mohanty, et al.), J. Phys. G38 (2011) 124023.

[78] J. Cleymans, H. Oeschler, K. Redlich and S. Wheaton, Phys. Lett. B615 (2005) 50-54.

[79] http://newscenter.lbl.gov/news-releases/2011/06/23/when-matter-melts/.

[80] M. Asakawa, U. Heinz, and B. Müller, Phys. Rev. Lett. 85 (2000) 2072.

[81] e.g. see V. Koch, PoS(CFRNC2006)008

[82] A. H. Mueller, Phys. Rev. D4 (1971) 150.

[83] Presentation by T. J. Tarnowsky at Quark Matter 2011, STAR Collab. (T. J. Tarnowsky et al.), J. Phys. G38 (2011) 124054.

[84] STAR Collab. (M. M. Aggarwal, et al.), Phys. Rev. Lett. 105 (2010) 022302.

[85] S. Gupta, X. Luo, B. Mohanty, H. G. Ritter and N. Xu, Science 332 (2011) 1525. 
[86] PHENIX Collab. (A. Adare, et al.), Phys. Rev. D85 (2012) 092004.

[87] STAR Collaboration (L. Adamczyk, et al.), Beam energy dependence of moments of the netcharge multiplicity distributions in $\mathrm{Au}+\mathrm{Au}$ collisions at RHIC (2014), arXiv:1402.1558.

[88] T. J. Tarnowsky and G. D. Westfall, Phys. Lett. B724 (2013) 51.

[89] O. E. Barndorff-Nielsen, D. G. Pollard and N. Shephard, Quant. Finance 12 (2012) 587-605.

[90] See R. Baier, D. Schiff and B. G. Zakharov, Ann. Rev. Nucl. Part. Sci. 50 (2000) 37-69, and references therein.

[91] H. Fritzsch and P. Minkowski, Phys. Lett. B69 (1977) 316.

[92] PHENIX Collab. (K. Adcox et al.), Phys. Rev. Lett. 88 (2002) 022301.

[93] PHENIX Collab. (S. S. Adler et al.), Phys. Rev. C76 (2007) 034904.

[94] PHENIX Collab. (A. Adare, et al.), Phys. Rev. Lett. 104 (2010) 132301.

[95] PHENIX Collab. (A. Adare, et al.), Phys. Rev. C87 (2013) 034911.

[96] ALICE Collab. (K. Aamodt, et al.), Phys. Lett. B696 (2011) 30.

[97] PHEnIX Collab. (A. Adare, et al.), Phys. Rev. Lett. 109 (2012) 152301.

[98] STAR Collab. (Xin Dong et al.), STAR Highlights, Quark Matter 2012, https://drupal.star.bnl.gov/STAR/files/STARhighlight_QM2012_0810.pdf

[99] PHENIX Collab. (A. Adare, et al.), Phys. Rev. C87 (2013) 054907.

[100] PHENIX Collab. (A. Adare, et al.), Phys. Rev. D86 (2012) 072008.

[101] L3 Collab. (B. Adeva et al.), Phys. Lett. B259 (1991) 199-208.

[102] PHENIX Collab. (S. S. Adler, et al.), Phys. Rev. D74 (2006) 072002.

[103] N. Borghini and U. A. Wiedemann, Nucl. Phys. A774 (2006) 549-522; see also arXiv:hepph/0506218v1.

[104] PHENIX Collab. (A. Adare et al.), Phys. Rev. D82 (2010) 072001.

[105] TASSO Collab. (W. Braunschweig et al.), Z. Phys. C47 (1990) 187.

[106] PHENIX Collab. (A. Adare et al.), Phys. Rev. Lett. 111 (2013) 032301.

[107] CMS Collab. (G. Roland et al.), Nucl. Phys. A904-905 (2013) 43c-50c.

[108] PHENIX Collab. (A. Adare et al.), Phys. Rev. Lett. 104 (2010) 252301.

[109] PHENIX Collab. (M. J. Tannenbaum et al.), arXiv:1109.0760v1 [nucl-ex],

[110] CMS Collab. (S. Chatrchyan et al.), Phys. Lett. B712 (2012) 176-197

[111] PHENIX Collab. (A. Adare et al.), Phys. Rev. Lett. 109 (2012) 122302.

[112] PHENIX Collab. (S. S. Adler, et al.), Phys. Rev. Lett. 94 (2005) 232301. 
[113] PHENIX Collab. (A. Adare et al.), Phys. Rev. Lett. 98 (2007) 172301.

[114] ALICE Collab. (B. Abelev et al.), JHEP 09 (2012) 112.

[115] CMS Collab. (S. Chatrchyan et al.), JHEP 05 (2012) 063.

[116] A. Zichichi, Nucl. Phys. A805 (2008) 36c.

[117] The Nobel Prize in Physics 2013, Nobelprize.org.

[118] PHENIX Collab. (R. Nouicer et al.), Nucl. Phys. A904-905 (2013) 647c-652c.

[119] PHENIX Collab. (A. Adare et al.), Phys. Rev. Lett. 98 (2007) 232301.

[120] PHENIX Collab. (T. Gunji et al.), J. Phys. G34 (2007) S749-S752, http://www.sinap.ac.cn/qm2006/parallel.htm\#p21.

[121] X. Zhao and R. Rapp, Phys. Lett. B664 (2008) 253-257

[122] P. Braun-Munzinger and J. Stachel, Phys. Lett. B490 (2000) 196-202.

[123] R. L. Thews, M. Schroedter and J. Rafelski, Phys. Rev. C63 (2001) 054905.

[124] A. Andronic, P. Braun-Munzinger, K. Redlich and J. Stachel, Nucl. Phys. A789 (2007) $334-$ 356.

[125] ALICE Collab. (B. Abelev, et al.), Phys. Rev. Lett. 109 (2012) 072301.

[126] ALICE Collab. (P. Giubellino, et al.), Highlights from ALICE, Presented at International School of Subnuclear Physics, 51st Course, Erice, Sicily, Italy, 24 June-3 July 2013, http: //wWw.ccsem.infn.it/issp2013/docs/Erice_Giubellino_2013.pdf

[127] R. L. Thews and M. L. Mangano, Phys. Rev. C73 (2006) 014904.

[128] H. Satz, Advances in H E P 2013 (2013) 242918. Also see arXiv:1303.3493v2

[129] PHENIX Collab. (A. Adare et al.), Phys. Rev. C84 (2011) 044905.

[130] STAR Collab. (L.Adamczyk et al.), Phys. Lett. B722 (2013) 55-62.

[131] PHENIX Collab. (A. Adare, et al.), Phys. Rev. C88 (2013) 024906.

[132] PHOBOS Collab. (B. B. Back, et al.), Phys. Rev. C72 (2005) 031901(R).

[133] A. Białas, A. Błeszynski and W. Czyż, Nucl. Phys. B111 (1976) 461.

[134] PHOBOS Collab. (B. B. Back, et al.), Phys. Rev. C74 (2006) 021901(R).

[135] M. Basile, ... A. Zichichi, et al., Phys. Lett. B95 (1980) 311-312.

[136] PHENIX Collab. (S. S. Adler et al.), Phys. Rev. C89 (2014) 044905.

[137] S. Eremin and S. Voloshin, Phys. Rev. C67 (2003) 064905.

[138] R. Nouicer, Eur. Phys. J. C49 (2007) 281-286.

[139] A. Białas, W. Czyż and L. Lesniak, Phys. Rev. D25 (1982) 2328. 
[140] ATLAS Collaboration, ATLAS-CONF-2013-096 (September, 2013).

[141] CMS Collab. (S. Chatrchyan, et al.), Phys. Lett. B718 (2013) 795.

[142] PHENIX Collab. (A. Adare, et al.), Phys. Rev. Lett. 111 (2013) 212301.

[143] PHENIX Collaboration, The sPHENIX MIE Proposal (2013) http://www.phenix.bnl.gov/ plans.html 